ADP-13-29/T849

IFT-UAM/CSIC-13-133

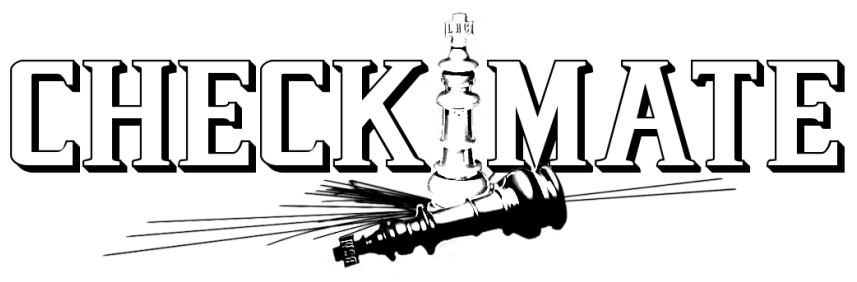

\title{
CheckMATE: Confronting your Favourite New Physics Model with LHC Data
}

\author{
Manuel Drees ${ }^{*}$ Herbi Dreiner $\dagger$ and Daniel Schmeier \\ Physikalisches Institut and Bethe Center for Theoretical Physics, University of Bonn, Bonn, Germany \\ Jamie Tattersal $\$$ \\ Institut für Theoretische Physik, University of Heidelberg, Heidelberg, Germany and \\ Physikalisches Institut and Bethe Center for Theoretical Physics, University of Bonn, Bonn, Germany \\ Jong Soo Kim T \\ Instituto de Fisica Teorica UAM/CSIC, Madrid, \\ Spain and ARC Centre of Excellence for Particle Physics at the Terascale, \\ School of Chemistry and Physics, University of Adelaide, Australia
}

(Dated: January 29, 2014)

In the first three years of running, the LHC has delivered a wealth of new data that is now being analysed. With over $20 \mathrm{fb}^{-1}$ of integrated luminosity, both ATLAS and CMS have performed many searches for new physics that theorists are eager to test their model against. However, tuning the detector simulations, understanding the particular analysis details and interpreting the results can be a tedious task.

Checkmate (Check Models At Terascale Energies) is a program package which accepts simulated event files in many formats for any model. The program then determines whether the model is excluded or not at $95 \%$ C.L. by comparing to many recent experimental analyses. Furthermore the program can calculate confidence limits and provide detailed information about signal regions of interest. It is simple to use and the program structure allows for easy extensions to upcoming LHC results in the future.

Checkmate can be found at: http://checkmate.hepforge.org

\footnotetext{
* drees@th.physik.uni-bonn.de

$\dagger$ dreiner@th.physik.uni-bonn.de

$\ddagger \overline{\text { daschm@th.physik.uni-bonn.de }}$

$\S$ j.tattersall@thphys.uni-heidelberg.de

ฯ jong.kim@csic.es
} 


\section{CONTENTS}

Important Note

I. Introduction

A. Motivation

B. Overview

II. Using Checkmate

III. Internal Details

A. Investigating the Results

8

B. Delphes Tunings

IV. Analyses

14

A. Analysis Code
B. Reference Data
C. Currently Validated Analyses

V. Performance Studies 17

A. Validation
B. Computing Performance

VII. Summary 20

Acknowledgements

A. Getting Started 21

B. Adding Analyses 23

1. Using the Analysis Manager 23

2. Analysis Code 23

C. ATLAS Detector Tunings 25

1. Jets and Photons

2. Electrons 26

3. Muons 27

4. B-Tagger 27

5. Tau-Tagger 29

D. Analysis Validation 34

1. atlas_conf_2012_104 34

2. atlas_conf_2012_147 35

3. atlas_conf_2013_024 36

4. atlas_conf_2013_035 38

5. atlas_conf_2013_047 39

6. atlas_conf_2013_049 42

7. atlas_conf_2013_061 44

8. atlas_conf_2013_089 46

9. cms_1303_2985 48

References 


\section{Important Note}

- Checkmate is built upon the tools and hard work of many people. If Checkmate is used in your publication it is extremely important that all of the following citations are included,

- Delphes 3 [1].

- FastJet [2, 3.

- Anti-kt jet algorithm [4].

- $\mathrm{CL}_{\mathrm{S}}$ prescription [5].

- In analyses that use the MT2 kinematical discriminant we use the Oxbridge Kinetics Library [6, 7, and the algorithm developed by Cheng and Han [8].

- All experimental analyses that were used to set limits in the study.

- The Monte Carlo event generator that was used.

\section{INTRODUCTION}

\section{A. Motivation}

In the three years that the Large Hadron Collider (LHC) has been running, the Beyond the Standard Model (BSM) landscape has changed enormously. To take just a few examples, the possible parameter spaces of Supersymmetry (SUSY) [9, 10, Technicolour [11, 12] and Extra Dimensional models [13, 14, are now significantly more limited and constrained than in the pre-LHC era.

LHC experimentalists typically classify analyses in terms of a specific final state configuration. They interpret the results in essentially two different approaches: The first strategy is to take a constrained version of a complete model, with a small number of free parameters and investigate exactly what set of model parameters have been excluded by the latest data and which are still allowed. The advantage of such a complete model is its predictivity and the small number of input parameters. However, as soon as the model is slightly modified, it can be very difficult, if not impossible, to set a new appropriate limit. Therefore, only a small number of models tested by the experimental collaborations can be reliably checked.

The second approach employed to great effect at the LHC uses simplified models [15]. The idea here is that an effective Lagrangian with only a small number of particles and interactions is considered. Limits on the mass of BSM particles, in particular production and decay topologies, are presented. The production and decay modes chosen are those that often occur in a particular class of models and consequently can be applied widely.

However, the particular interpretation of these results can lead to very different results. For example, the headline limits are often presented in terms of $100 \%$ branching ratios and in models with a dark matter candidate [16], this is often taken as being massless. In most 'realistic' models, neither of these assumptions is true which can lead to a far more conservative bound.

One may nevertheless attempt to map the particular model of interest onto the simplified model by considering the rate of events that have exactly the same production and decay topologies. However, rarely are branching ratios $100 \%$ and many different production modes may contribute. These different topologies may not be identical to those given in the simplified model and so cannot be safely used to set a limit. Despite this, it is inevitable that some events from these processes will contribute to signal regions. Consequently, the limit set may be far weaker than if the full model had been properly analysed by the particular experimental collaboration.

The above two issues lead to the obvious question of how to reinterpret the data to place bounds on the BSM models that have not previously been analysed by the experiments. Unavoidably, the only way to do this accurately is to generate simulated events and then run these through an implementation of the experimental analysis, including all detector effects. Checkmate is meant to provide the best possible reproduction of both the analysis and detector effects. In addition we aim to simplify and improve confidence in the second step so that any user can investigate the model of their choice.

The idea behind CheckmaTe is that the user only has to provide event file(s) and the corresponding cross section(s). The user also selects the analyses 11 against which the event files should be tested. Checkmate then informs the user whether the particular model is excluded or not using the $95 \% \mathrm{CL}_{\mathrm{S}}$ [5] method. In addition, the actual exclusion likelihood can be given if desired.

\footnotetext{
${ }^{1}$ If the particular analysis of interest is missing from the current Checkmate list, we welcome requests for new analyses. Indeed, the analysis under question may be almost ready for public release and is only awaiting final validation.
} 


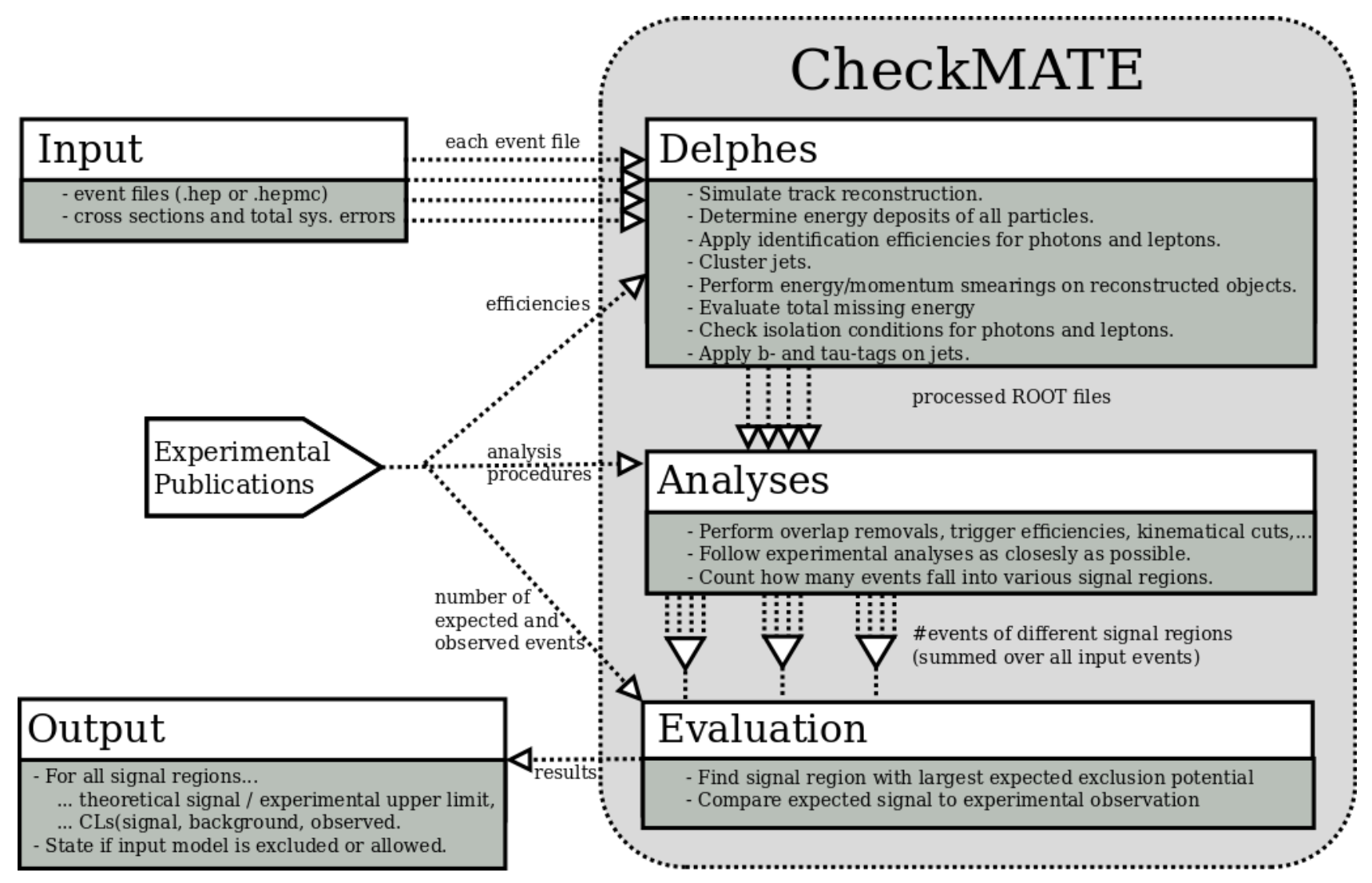

FIG. 1: Flow chart to demonstrate the chain of data processing within CheckMATE.

\section{B. Overview}

To run Checkmate, the user has to provide simulated event files (in HepMC 17 or HepEvt 18]) from an event generator of choice (Pythia [19, 20], Herwig ++ [21], CalcHEP 22], MadGraph [23], Sherpa [24], ...) plus the effective cross sections that correspond to these events, e.g. taking into account potential phase space cuts or branching ratios. The total systematic $1 \sigma$ error on the cross section has to be provided which enters the calculation of the total uncertainty. Input events can be weighted and these weights are properly taken into account by CheckMATE.

This information is then consecutively processed by three independent modules (see Figure 1). As a first step, the events are run through a fast detector simulation. We have chosen to use Delphes [1], which we have heavily modified to accurately model the ATLAS detector and the final state reconstructions that are performed. This includes the smearing of the visible particles' momenta according to the respective experimental resolution, efficiency-based object identification, $\mathrm{b}-/ \tau$-tagging for jets and reconstruction of the missing transverse momentum vector. The program has been optimised to check for all the final state information needed by the given selection of analyses and to minimise the computational effort by removing redundant and/or superfluous parts from the simulation, see Section III B for more details. The results of this simulation are stored in Root trees, which can optionally be read and/or manipulated by the user externally [25].

These files are automatically processed by those of the supplied analyses the user selects when starting CheckMATE. A list of currently implemented analyses can be found in Table I and will be significantly extended in the coming months. They are internally coded in a well-structured framework that allows for an easy extension to new upcoming experimental results (see Appendix $\mathrm{B}$ for more details). It furthermore allows users to easily update given analyses or implement their own.

The input data is processed event by event by checking isolation criteria, removing overlapping objects, and implementing the cuts that define the signal regions. The analysis program determines how many events in total satisfy certain signal region criteria and stores this information in human-readable output for each separate input event file. Both the true number of Monte Carlo events as well as the number of events normalised to the given cross section and luminosity are stored.

The final step of the program consists of a statistical evaluation of the result. For each individual signal region of 


\begin{tabular}{|c|c|c|c|c|c|c|}
\hline Internal name & \#SR & Description & $\sqrt{s}$ & $\mathcal{L}$ & Ref & CR? \\
\hline atlas_1308_2631 & 2 & 2 b-jets $+\mathbb{E}_{T}$ & $8 \mathrm{TeV}$ & $20.1 \mathrm{fb}^{-1}$ & 26 & $\checkmark$ \\
\hline atlas_conf_2012_104 & 2 & 1 lepton $+\geq 4$ jets $+\mathbb{E}_{T}$ & $8 \mathrm{TeV}$ & $5.8 \mathrm{fb}^{-1}$ & 27 & \\
\hline atlas_conf_2012_147 & 4 & Monojet $+\bar{E}_{T}$ & $8 \mathrm{TeV}$ & $10.5 \mathrm{fb}^{-1}$ & 28 & $\checkmark$ \\
\hline atlas_conf_2013_024 & 3 & 0 lepton $+6(2 \mathrm{~b}$ - $)$ jets $+\mathbb{E}_{T}$ & $8 \mathrm{TeV}$ & $20.5 \mathrm{fb}^{-1}$ & 29 & $\checkmark$ \\
\hline atlas_conf_2013_035 & 6 & 3 leptons $+\mathbb{E}_{T}$ & $8 \mathrm{TeV}$ & $20.7 \mathrm{fb}^{-1}$ & 30 & \\
\hline atlas_conf_2013_047 & 9 & 0 leptons $+2-6$ jets $+\mathbb{E}_{T}$ & $8 \mathrm{TeV}$ & $20.3 \mathrm{fb}^{-1}$ & 31 & $\checkmark$ \\
\hline atlas_conf_2013_049 & 9 & 2 leptons $+\mathbb{E}_{T}$ & $8 \mathrm{TeV}$ & $20.3 \mathrm{fb}^{-1}$ & 32 & \\
\hline atlas_conf_2013_061 & 9 & 0-1 leptons $+\geq 3$ b-jets $+\mathbb{E}_{T}$ & $8 \mathrm{TeV}$ & $20.1 \mathrm{fb}^{-1}$ & 33 & $\checkmark$ \\
\hline atlas_conf_2013_062 & 19 & $1-2$ leptons $+\overline{3}-6$ jets $+\mathbb{E}_{T}$ & $8 \mathrm{TeV}$ & $20.3 \mathrm{fb}^{-1}$ & 34 & \\
\hline atlas_conf_2013_089 & 12 & 2 leptons + jets $+\mathbb{E}_{T}$ & $8 \mathrm{TeV}$ & $20.3 \mathrm{fb}^{-1}$ & 35 & $\checkmark$ \\
\hline cms_pas_exo_12_048 & 7 & Monojet & $8 \mathrm{TeV}$ & $19.5 \mathrm{fb}^{-1}$ & 36 & \\
\hline cms_1303_2985 & 59 & $\alpha_{T}+\mathrm{b}$ jet multiplicity & $8 \mathrm{TeV}$ & $11.7 \mathrm{fb}^{-1}$ & 37 & $\checkmark$ \\
\hline
\end{tabular}

TABLE I: List of currently available analyses in CheckMATE. '\#SR' shows the number of signal regions the respective analysis provides. The rightmost column shows whether control regions for that particular analysis have been implemented or not.

the chosen analyses, the total number of expected signal events $S$ is determined by summing up the results from each input event file. The total $1 \sigma$ uncertainty $\Delta S$ on this number is determined from both the statistical uncertainty, given by the number of Monte Carlo events, and the systematic uncertainty, which is estimated from the total uncertainty on the signal cross section given by the user. These numbers are compared to the results from the respective experimental search, listed in the references shown in Table 1 There are two possible ways of comparison:

1. Many experimental searches translate their results into model independent $95 \%$ upper confidence limits on the number of signal events coming from new physics. A quick-and-easy way of comparison is given by computing the parameter,

$$
r \equiv \frac{S-1.96 \cdot \Delta S}{S_{\text {Exp. }}^{95}}=\frac{95 \% \text { lower limit on the number of signal events, determined by CheckMATE }}{\text { Experimentally measured } 95 \% \text { confidence limit on signal events }} .
$$

In that case, a model can be considered as excluded to the $95 \%$ confidence level, if $r \geq 1$.

2. The user can ask for the explicit confidence level for the given signal. Following common use of experimental searches, Checkmate uses a profile $\log$-likelihood ratio test paired with the $\mathrm{CL}_{\mathrm{S}}$ prescription $[5$ to determine the confidence level that corresponds to the determined number of signal events and the expected and observed number of events at the experiment. Both the uncertainty on the signal and on the background are taken into account as Gaussian probability distribution functions around the nominal values. The calculation of the $\mathrm{CL}_{\mathrm{S}}$ allows for CheckMATE to be easily included into a fitting routine for the chosen model.

The aforementioned parameters are determined for all signal regions of all selected analyses. If available from the experimental papers, not only the observed but also the expected limits are used. The signal region with the strongest expected limit is determined and the corresponding observed limit is used to state whether the input can be considered excluded or not.

\section{USING CHECKMATE}

This section introduces the basic setup of the program. It should give the users enough insight into the program to run the code for their own purposes, which does not require knowledge of the internal details. These will be introduced in Section III B and the Appendices. An even simpler version showing the minimum required information to run Checkmate and extract the relevant results is given on the Checkmate website. Checkmate can easily be downloaded and installed ${ }^{2}$ by the following set of commands:

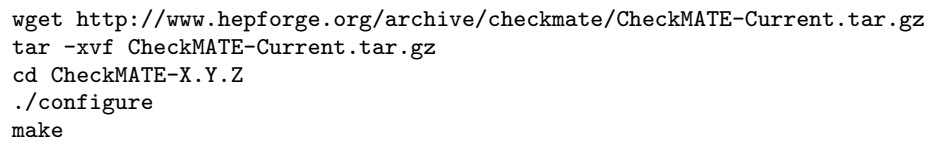

${ }^{2}$ If you encounter problems during installation, please contact us via the webpage and we are happy to help. 
More information on Root prerequisites and installation parameters can be found in Appendix A. A successful installation should have created a CheckMATE binary within the bin/ folder of the installation directory.

To run Checkmate, the user has to provide at least the following information:

Name of the run: A unique name has to be set to define the current run and to set up the output folder accordingly.

Analyses to be tested: The user has to choose at least one of the provided analyses to be used by CheckMATE for testing the input data against experiment. Any combination of the available analyses can be chosen. Note that a single analysis usually defines several signal regions, all of which will be investigated by CheckMATE.

Events: Any number of event files can be given as long as they are either in .hep, .hepmc or .lh $4^{3}$ format, which allows linkage to all modern event generators. Weighted event files are allowed and properly considered within the detector simulation, the analyses and the evaluation.

Processes: In case there are multiple input event files, they have to be sorted into processes, since Checkmate needs to know whether they correspond to the same or to different production modes. This will change the final summation over all individual results; see Footnote 7 for more details

Total cross section and error: In order to properly normalise the final number of events in each signal region to the corresponding luminosity of the respective experimental data, the total cross section that corresponds to a given process has to be provided. Furthermore, an absolute error on that value is necessary to quantify the effect of this systematic uncertainty on the final exclusion. Note that all event files for a given process (see above) are considered to have identical cross sections, i.e. they must have been created with the same event generator settings.

In addition to the aforementioned list, several optional parameters are available. They are listed and explained in Table II] Checkmate provides two possibilities to let the user define the options of a certain run:

Parameter File: A convenient method to provide the parameters of choice by the user is the usage of a separate text-file that stores all information in human readable form. A minimal example looks as follows:

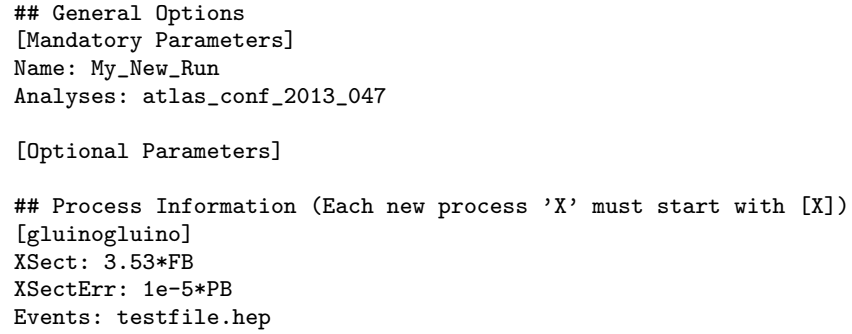

Parameter names and corresponding values have to be separated by a colon. Most optional parameters need a Boolean answer; they can be either True/False, Yes/No, 1/0 or on/off. The items Analyses and Events can consist of more than one entry. In that case, they have to be separated by commas (line breaks are allowed as long as the new lines start with a whitespace). Every separate process has to be introduced with a new [PROCESSNAME] block and has to contain the three entries XSect, XSectErr and Events as shown in the above example.

In order to load CheckMATE via such an input parameter file, the user has to run ./CheckMATE PARAMETERFILE.

Command Line Input: For an automatised embedding of CheckmATE into a program chain, it can be useful if one is not obliged to save the parameters in a separate file. For that purpose, all parameters mentioned before and in Table II can also be directly entered into CheckmaTe in the form of command line parameters. The above example would look as follows:

bin/: ./CheckMATE -n My_New_Run -a atlas_conf_2013_047 -p "gluinogluino" -xs "3.53*FB" -xse "1e-5*PB" testfile.hep

\footnotetext{
3 The use of lhe event files is strongly discouraged. If they have been created from a parton level Monte-Carlo program, the lack of a parton shower can significantly alter the analysis acceptances and lead to incorrect limits. Even if the events have been showered, the choice of jet clustering before a detector simulation has been performed can lead to large differences. If lhe files are required care must be taken when employing analysis that make use of flavour tagging. For flavour tagging to be performed, the parton level particles must be stored in the event file along with the final state particles.
} 


\begin{tabular}{|c|c|c|}
\hline Input File & Command Line & Definition \\
\hline FullCL & $-c l,--f u l l-c l$ & $\begin{array}{l}\text { Calculates } \mathrm{CL}_{\mathrm{S}} \text { explicitly for each signal region and uses it for the exclusion } \\
\text { statement. }\end{array}$ \\
\hline RandomSeed & $-r s,--r a n d o m-s e e d$ & Defines a fixed seed for the random number generator. \\
\hline OutputDirectory & -od, --outdir & $\begin{array}{l}\text { This defines the path to the directory into which all CheckMATE output should } \\
\text { be saved. Both absolute and relative paths are allowed. If not set, CheckMATE } \\
\text { will create and use a results / directory within its main folder. }\end{array}$ \\
\hline OutputExists & -oe, --output-exists & $\begin{array}{l}\text { If there are already output files with the same run name in the same output } \\
\text { directory as the current run, CheckMATE can deal with this in different ways: } \\
\text { overwrite will delete the old output and restart with a new run. add will consider } \\
\text { the current run as an addendum to the previous run and will add the current } \\
\text { results to the old ones. overwrite will always ask the user via prompt. }\end{array}$ \\
\hline SkipParamCheck & -sp, --skip-paramcheck & Skip startup parameter check. \\
\hline SkipDelphes & -sd, --skip-delphes & $\begin{array}{l}\text { Only works if the output directory already exists and has Delphes RoOT files. } \\
\text { Delphes won't run and the given RooT files in the output directory are re- } \\
\text { processed by the given analyses. This is only useful in case the user changed } \\
\text { parts of the analysis and wants to test with the same input. }\end{array}$ \\
\hline SkipEvaluation & -se, --skip-evaluation & $\begin{array}{l}\text { The input files are only processed by the detector simulation and the chosen } \\
\text { analyses, but the analysis result will not be further evaluated and compared to } \\
\text { experimental data. It should be used when doing a control region analysis or for } \\
\text { debugging purposes. }\end{array}$ \\
\hline QuietMode & $-q,--q u i e t$ & No output will be print on screen. \\
\hline VerboseMode & $-v,--v e r b o s e$ & All Delphes and analysis output will be printed to the standard output. \\
\hline TempMode & -t, --temporary & $\begin{array}{l}\text { All Delphes Root files will be deleted after the analysis step to save hard disk } \\
\text { space. }\end{array}$ \\
\hline
\end{tabular}

TABLE II: List of all currently available optional parameters for CheckmATE. The first column denotes the string to be used in the parameter card; it has to be added as a separate line in the [Optional Parameters] block as PARAMETER = VALUE. Boolean values can be given as any of the following: True/False, Yes/No, 1/0 or on/off. The standard value for all these is No. The second column names the corresponding parameter to be used in the command line input in both a short and an extended format. They can be used by adding -PARAMETER=VALUE or -PARAMETER to set Boolean parameters to True.

Note that the list of event files comes last without any prefix indicator and separated by spaces only. In case of $N$ event files, there have to be either exactly 1 or exactly $N$ entries in the options for $-\mathrm{p}$, -xs and -xse which have to be separated by semicolons. If there is only one entry, CheckMATE will assume that all given event files belong to the same process with identical cross sections and errors. Otherwise it will consider event file $i$ to belong to the process at the $i$ th position etc.

Running either of the aforementioned examples with the event file testfile.hep provided by the CheckMATE installation should, after confirmation and some intermediate output, produce the following result (which should not be surprising as the test events effectively do not contain any signal events):

$[\ldots]$

Test: Calculation of $\mathrm{r}=$ signal/(95\%CL limit on signal)

Result: Allowed

Result for $r_{\text {: }} r_{-} \max =0.0$

SR: atlas_conf_2013_047 - AL

The most important result is given by the Result: line, which will either state Allowed or Excluded. This statement alone allows the user to quickly perform simple tests on his model of interest. However, if they should be interested in the intermediate details that lead to this result, they can find further information in Section IIIA. 


\section{INTERNAL DETAILS}

\section{A. Investigating the Results}

In order to demonstrate the structure of CheckMATE's output, we show and discuss the results for a reasonably complex example. We will describe the steps that CheckMATE performs in detail and discuss the information that can be extracted from the results. Knowing these details allows use of CheckMATE for more detailed studies and/or to adapt the code to the user's needs.

\section{Description of the Example Run}

Input events have been produced with MadGraph using the CMSSM model with $m_{0}=2000 \mathrm{GeV}, m_{1 / 2}=$ $450 \mathrm{GeV}, \tan \beta=30, A_{0}=-4000 \mathrm{GeV}$ and $\mu>0$. The total cross sections for $\tilde{g} \tilde{g}$ and $\tilde{g} \tilde{q}$ production at $p p$ collisions at $\sqrt{s}=8 \mathrm{TeV}$ have been estimated with NLL-Fast 38 43, together with the respective theoretical uncertainties from scale variations and PDF dependences, to be $\sigma_{\tilde{g} \tilde{g}}=(5.52 \pm 0.68) \mathrm{fb}$ and $\sigma_{\tilde{g} \tilde{q}}=(0.926 \pm 0.173)$ fb. Squark pair production has been neglected 4 . For each production process, we created two event files with 10000 events each, which we desire to be tested against atlas_conf_2013_047, i.e. a zero lepton search with missing energy and two to six jets. The $\mathrm{CL}_{\mathrm{S}}$ value should be used for the final exclusion statement. The corresponding input parameter file is shown in Figure 2 .

\section{Structure of the Results Folder}

A successful run with the parameter file shown in Figure 2 should have created a results folder at the desired destination. It consists of the following files and directories:

/hdd/results/cMSSM: ls

analysis delphes evaluation progress.txt result.txt

To keep a general overview on all processed data files, CheckMATE produces a file progress.txt at the top level of the results directory. It lists the cross section and process information of all events files that have been fully analysed by CheckmATE, together with the time of production and a unique prefix that identifies the output files which correspond to a given input file. In the aforementioned example, the progress file would look as follows:

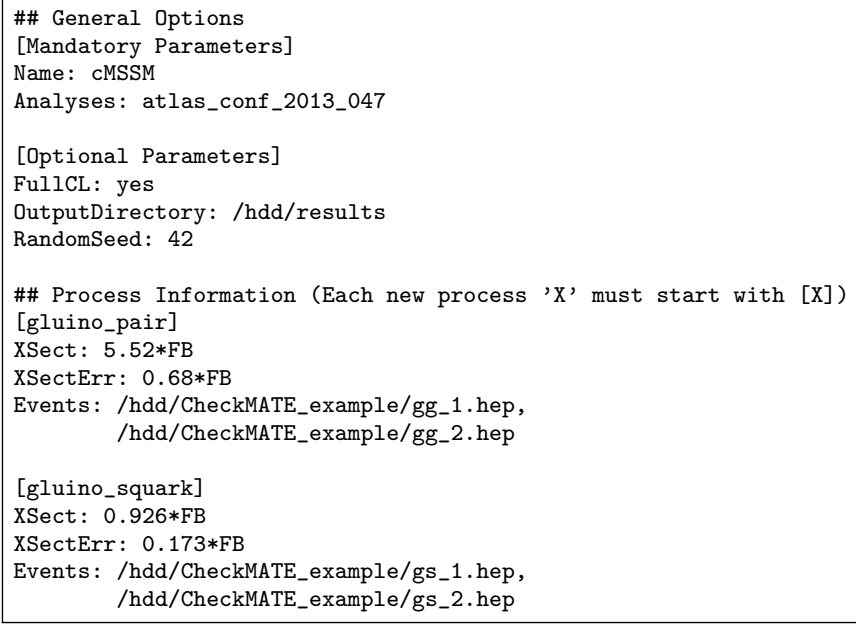

FIG. 2: Parameter input file that corresponds to the example scenario described in the text.

\footnotetext{
${ }^{4} m_{\tilde{q}}=2.17 \mathrm{TeV}, \sigma_{\tilde{q} \tilde{q}} \approx 0.02 \mathrm{fb}$
} 


\begin{tabular}{|c|c|c|c|c|c|}
\hline \#Prefix & EventFile & Sigma & dSigma & Process & Date and Time of Procession \\
\hline 000 & /hdd/CheckMATE_example/gg_1.hep & $5.52 \mathrm{FB}$ & $0.68 \mathrm{FB}$ & gluino_pair & $2013-11-28 \quad 13: 29: 46.981908$ \\
\hline 001 & /hdd/CheckMATE_example/gg_2.hep & $5.52 \mathrm{FB}$ & $0.68 \mathrm{FB}$ & gluino_pair & $2013-11-28 \quad 13: 30: 43.669558$ \\
\hline 002 & /hdd/CheckMATE_example/gs_1.hep & $0.926 \mathrm{FB}$ & $0.173 \mathrm{FB}$ & gluino_squark & $2013-11-28 \quad 13: 31: 52.322075$ \\
\hline 003 & /hdd/CheckMATE_example/gs_2.hep & $0.926 \mathrm{FB}$ & $0.173 \mathrm{FB}$ & gluino_squark & $2013-11-28 \quad 13: 32: 59.544565$ \\
\hline
\end{tabular}

This file is particularly important when the user uses the add (see Table II for more details) feature of CheckmaTE, i.e. when they add data to an already existing output directory, as all necessary information from previous runs is stored in the above shown progress file.

\section{Delphes}

As a first step, the input event file has to be processed by the detector simulation Delphes, which will produce the following output:

/hdd/results/cMSSM: ls delphes

000_delphes.root 001_delphes.root 002_delphes.root 003_delphes.root log_delphes.txt merged.tcl

First, the simulation has to be set up according to the required information the later analyses need (see Footnote 10 . This so-called detector-card, which saves the settings in a specific syntax the Delphes code is able to parse internally, is generated at the beginning of the CheckMATE run and is saved in /delphes/merged.tcl for later inspection by the user. All output that is generated by Delphes will be stored in a single log file, log_delphes.txt and can be examined later; this can e.g. help to understand unexpected behaviour of the simulation.

Furthermore, Checkmate will keep all Root trees Delphes generates and stores them in the same folder. They can be investigated directly by the user, e.g. by using the RoOT internal TBrowser object or indirectly by the subsequent analysis step. The naming of the individual files uses the prefix mapping as listed in the previously mentioned progress .dat. There will always be one Rоoт file per input event file, irrespective of the number of chosen analyses.

It should be noted that, depending on the number and complexity of the chosen analyses and the size of the input event files, the resulting Rоот trees can be very large and the corresponding file might consume large amounts of disk space 5 . To avoid this, the user can declare that these files should be immediately deleted after the analysis step by using the tempMode setting in Checkmate (see Table II). Still, the complex Root structure needs the output of one Delphes run to be saved as one complete file, i.e. it is impossible to pipe the data on the fly from Delphes to the analysis code. The user therefore has to make sure that enough disk space is available to save at least one full RoOT file, even when tempMode is activated. Should disk space be very limited, the user might consider splitting the input event file into multiple subfiles.

\section{Analysis}

Looking into the analysis folder reveals the following files:

/hdd/results/cMSSM: Is analysis

000_atlas_conf_2013_047_cutflow.dat 002_atlas_conf_2013_047_cutflow.dat 000_atlas_conf_2013_047_signal.dat 002_atlas_conf_2013_047_signal.dat 001_atlas_conf_2013_047_cutflow.dat 003_atlas_conf_2013_047_cutflow.dat 001_atlas_conf_2013_047_signal.dat 003_atlas_conf_2013_047_signal.dat

log_analysis.txt

The Rоoт files produced by Delphes will automatically be processed by all the chosen analyses. Each input file will create its individual outputs, identified by the prefix defined in the progress file. Most analyses that are included in Checkmate will produce only two types of output: cutflow-files show the absolute and relative amounts of data that pass the individual event selection cuts of the corresponding analysis, whereas signal-files give the amount of events that fall into each signal region defined by the analysis. The latter file is more important for CheckMATE as its content is used for the later evaluation step. The cutflow information can be used by the user for various purposes (e.g. validating analyses by comparing with the cutflows produced by the collaborations), but it is not needed by Checkmate.

\footnotetext{
5 The size of the Rоoт file depends upon the type of signal events produced (e.g. events with lots of hard jets give larger files), the particular Monte-Carlo program used to generate the events and the particular analysis being run. As a rough estimate however, 1000 events should produce a Root file size below $20 \mathrm{Mb}$.
} 
Examples for both types of output are shown in Figure 3 After some general information on the analysi: ${ }^{6}$ and the processed event file, a list of all individual cutflow milestones / signal regions follows. For each of these, Checkmate lists the sum of weights (and squared weights) of all events that passed the corresponding cut(s) (Sum_W, Sum_W2), the relative number compared to the total sum of weights (Acc) as well as the physically expected number of events (N_Norm), after normalising to the given total cross section of the data and the luminosity of the respective analysis. In case of unweighted events, Sum_W and Sum_W2 corresponds to the number of Monte Carlo events in the respective region. However, in case of weighted events, both these columns are needed by Checkmate to properly calculate the statistical error in the upcoming evaluation step.

We note that usually the analyses do not produce any output on the screen but just write into the above described output files. However, in case of error: ${ }^{7}$ and/or if the user adds extra print commands to the analysis code, the output will be stored in log_analysis.txt.

\section{Evaluation}

We discuss the last step, namely examining the evaluation folder:

/hdd/results/cMSSM: Is evaluation

atlas_conf_2013_047_cl_limits.txt atlas_conf_2013_047_event_numbers.txt atlas_conf_2013_047_r_limits.txt best_signal_regions.txt

In the evaluation step, the results of all individual analyses have to be combined to compare the total number of events to the experimental limits. The result of this summation is stored in evaluation/ANALYSISNAME_event_numbers.txt (as an example, see Figure 4) and utilises the following logic. Using the information from the progress file, CheckMATE will determine which *_signal.dat files correspond to the same and which to different input processes. Events that correspond to the same process will be considered as if they originated from one large input file, i.e. their results will be averaged by taking the corresponding weights properly into account. The statistical error is calculated from the

\begin{tabular}{|c|c|c|c|c|}
\hline \multicolumn{5}{|c|}{$\begin{array}{l}\# \text { ATLAS } \\
\# \text { ATLAS-CONF-2013-047 } \\
\# \text { O leptons, } 2-6 \text { jets, etmiss } \\
\# \text { sqrt }(\mathrm{s})=8 \mathrm{TeV} \\
\# \operatorname{int}(\mathrm{L})=20.3 \mathrm{fb}^{-}-1\end{array}$} \\
\hline \multicolumn{3}{|c|}{ Inputfile: } & \multicolumn{2}{|c|}{ /hdd/results/cMSSM/delphes/000_delphes.root } \\
\hline \multicolumn{3}{|c|}{ XSect : } & \\
\hline \multicolumn{3}{|c|}{ Error: } & \multicolumn{2}{|l|}{$\begin{array}{l}5.52 \mathrm{fb} \\
0.68 \mathrm{fb}\end{array}$} \\
\hline \multicolumn{3}{|c|}{ MCEvents : $\quad 1$} & \multicolumn{2}{|l|}{10000} \\
\hline \multicolumn{5}{|c|}{ Sum0fWeights: 10000} \\
\hline \multicolumn{5}{|c|}{ Sum0fWeights2: } \\
\hline \multicolumn{5}{|c|}{ NormEvents: } \\
\hline Cut & Sum_W & Sum_W2 & 2 Acc & N_Norm \\
\hline 0 & 10000 & 10000 & 1 & 111.608 \\
\hline 1 & 4439 & 4439 & 0.4439 & 49.5427 \\
\hline 2 & 3694 & 3694 & 0.3694 & 41.2279 \\
\hline 3 & 3680 & 3680 & 0.368 & 41.0717 \\
\hline 3J1 & 3676 & 3676 & 0.3676 & 41.027 \\
\hline 3J2 & 3048 & 3048 & 0.3048 & 34.0181 \\
\hline 4 & 3680 & 3680 & 0.368 & 41.0717 \\
\hline & 3622 & 3622 & 0.3622 & 40.4243 \\
\hline $\begin{array}{l}4 \mathrm{~J} 2 \\
{[\ldots]}\end{array}$ & 3002 & 3002 & 0.3002 & 33.5047 \\
\hline
\end{tabular}

\begin{tabular}{|c|c|c|c|c|}
\hline \multicolumn{5}{|c|}{$\begin{array}{l}\# \text { ATLAS } \\
\# \text { ATLAS-CONF-2013-047 } \\
\# \text { O leptons, } 2-6 \text { jets, etmiss } \\
\# \text { sqrt(s) }=8 \mathrm{TeV} \\
\# \operatorname{int}(\mathrm{L})=20.3 \mathrm{fb}^{\wedge}-1\end{array}$} \\
\hline \multicolumn{3}{|c|}{ Inputfile: } & \multicolumn{2}{|c|}{ /hdd/results/cMSSM/delphes/000_delphes.root } \\
\hline \multicolumn{3}{|c|}{ XSect: } & \\
\hline \multicolumn{3}{|c|}{ Error: } & \multicolumn{2}{|l|}{$\begin{array}{l}5.52 \mathrm{fb} \\
0.68 \mathrm{fb}\end{array}$} \\
\hline \multicolumn{3}{|c|}{ MCEvents : } & \multicolumn{2}{|l|}{10000} \\
\hline \multicolumn{3}{|c|}{ Sum0fWeights: } & \multicolumn{2}{|l|}{10000} \\
\hline \multicolumn{3}{|c|}{ SumOfWeights2: } & \multicolumn{2}{|l|}{10000} \\
\hline \multicolumn{3}{|c|}{ NormEvents: } & \multicolumn{2}{|l|}{111.608} \\
\hline $\mathrm{SR}$ & Sum_W & Sum_W2 & Acc & N_Norm \\
\hline $\mathrm{AL}$ & 2866 & 2866 & 0.2866 & 31.9868 \\
\hline AM & 353 & 353 & 0.0353 & 3.93975 \\
\hline BM & 478 & 478 & 0.0478 & 5.33485 \\
\hline BT & 35 & 35 & 0.0035 & 0.390627 \\
\hline CM & 1416 & 1416 & 0.1416 & 15.8037 \\
\hline CT & 89 & 89 & 0.0089 & 0.993309 \\
\hline D & 870 & 870 & 0.087 & 9.70988 \\
\hline EL & 1655 & 1655 & 0.1655 & 18.4711 \\
\hline EM & 1255 & 1255 & 0.1255 & 14.0068 \\
\hline ET & 609 & 609 & 0.0609 & 6.79691 \\
\hline
\end{tabular}

FIG. 3: Example analysis output for one particular input event file. The left figure shows the first lines of the cutflow file analysis/000_atlas_conf_2013_047_cutflow.dat. It first shows general information for the whole event file, like the total number of events, the sum of their weights, the sum of their squared weights and the number of physical events given by cross section $\times$ integrated luminosity. It follows a list of all cutflow levels with the sum of all weights (and squared weights) of the events that passed the corresponding cuts, the acceptance relative to the total sum of weights of the file and the number of physical events given by acceptance $\times$ cross section $\times$ integrated luminosity. The right figure shows the full signal result file analysis/000_atlas_conf_2013_047_signal.dat, which gives the the same information in the individual signal regions.

\footnotetext{
6 This header information is printed in all output files, but has been removed from all further example output files in this section.

7 Warnings '** WARNING: cannot access branch 'Jet2', return NULL pointer' might appear in the analysis log file. These are inevitably produced internally by Rоот but do not hint to any problems and should be ignored by the user.
} 


\begin{tabular}{|c|c|c|c|c|c|c|c|c|c|c|c|c|c|c|c|c|c|c|c|c|}
\hline Prefix & N_TotMC & $\mathrm{AL}$ & stat & sys & $\mathrm{AM}$ & stat & sys & BM & stat & sys & BT & stat & sys & CM & stat & sys & $\mathrm{CT}$ & stat & sys & . \\
\hline 000 & 10000.00 & 31.99 & 0.60 & 3.94 & 3.94 & 0.21 & 0.49 & 5.33 & 0.24 & 0.66 & 0.39 & 0.07 & 0.05 & 15.80 & 0.42 & 1.95 & 0.99 & 0.11 & 0.12 & $\ldots$ \\
\hline 001 & 10000.00 & 32.84 & 0.61 & 4.04 & 4.04 & 0.21 & 0.50 & 5.49 & 0.25 & 0.68 & 0.41 & 0.07 & 0.05 & 16.91 & 0.43 & 2.08 & 1.08 & 0.11 & 0.13 & . \\
\hline Tot & 20000.00 & 32.41 & 0.43 & 3.99 & 3.99 & 0.15 & 0.49 & 5.41 & 0.17 & 0.67 & 0.40 & 0.05 & 0.05 & 16.36 & 0.30 & 2.01 & 1.04 & 0.08 & 0.13 & . \\
\hline \multicolumn{21}{|c|}{ Process: gluino_squark } \\
\hline 002 & 10000.00 & 4.99 & 0.10 & 0.93 & 1.34 & 0.05 & 0.25 & 1.92 & 0.06 & 0.36 & 0.47 & 0.03 & 0.09 & 1.89 & 0.06 & 0.35 & 1.31 & 0.05 & 0.24 & . \\
\hline 003 & 10000.00 & 5.00 & 0.10 & 0.93 & 1.43 & 0.05 & 0.27 & 1.92 & 0.06 & 0.36 & 0.42 & 0.03 & 0.08 & 1.84 & 0.06 & 0.34 & 1.27 & 0.05 & 0.24 & $\ldots$ \\
\hline Tot & 20000.00 & 4.99 & 0.07 & 0.93 & 1.38 & 0.04 & 0.26 & 1.92 & 0.04 & 0.36 & 0.44 & 0.02 & 0.08 & 1.86 & 0.04 & 0.35 & 1.29 & 0.03 & 0.24 & . . \\
\hline \multicolumn{21}{|c|}{ 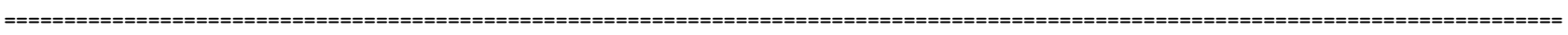 } \\
\hline Tot & 40000.00 & 37.41 & 0.43 & 4.10 & 5.37 & 0.15 & 0.56 & 7.33 & 0.18 & 0.76 & 0.84 & 0.05 & 0.10 & 18.22 & 0.30 & 2.04 & 2.32 & 0.08 & 0.27 & . \\
\hline
\end{tabular}

FIG. 4: Example result of the event summation routine during CheckMATE's evaluation step from

evaluation/atlas_conf_2013_047_event_numbers.txt. For each signal region, the final event numbers are averaged for events from the same and added for events in different processes. The errors are combined accordingly.

sum of weights and sum of squared weights in the given signal region, whereas the systematic error is given as $\delta \sigma / \sigma$ times the number of signal events after cuts, where $\sigma$ and $\delta \sigma$ are the cross section and error given as input by the user for the process the events correspond to (see Section III). Subsequently, results from different processes will contribute individually and therefore will be added to determine the total expected number of signal events. The errors are considered independent and hence added in quadrature. This is done for each signal region in each selected analysis separately.

These results are then compared to the experimental limits, separately for each signal region in each selected analysis. The conservative $r$-limit as defined in Equation (1) is always evaluated and the results are stored in evaluation/ ANALYSISNAME_r_limits.txt, together with the total number of signal events after cuts, the errors and the experimental upper limits. An example is shown in Figure 5 for the above example run.

If the FullCLs option has been activated in the input parameters, CheckmaTE will furthermore calculate the $\mathrm{CL}_{\mathrm{S}}$ value, using the total number and uncertainty of signal events determined by CheckMATE, the total number and uncertainty of expected background events and the number of observed events, all taken from the respective experimental source. The expected limit is determined by setting the 'observed' number of events in the signal region equal to the expected background $(\mathrm{O}=\mathrm{B})$. Since $\mathrm{CL}_{\mathrm{S}}$ is determined numerically, the result has a computational uncertainty that is also printed in the output to state the numerical stability of the result. All these numbers are stored in evaluation/ANALYSISNAME_cl_limits.txt.

As the last step, Checkmate will search for the signal region with the largest expected sensitivity; for the $r$-limits this corresponds to the signal region with the largest $r^{\wedge}{ }^{\wedge}$ exp, whereas for the $\mathrm{CL}_{\mathrm{S}}$ test the one with the smallest CL_exp is chosen. The results of the most sensitive signal region of each analysis separately is written in the file best_signal_regions.txt and an example is shown in Figure 7. Checkmate then chooses the most sensitive region among these and the corresponding observed result will be used to finally conclude whether the input can be considered to be excluded or not, i.e. in the case of the $r$-limit if $r^{\wedge} c_{-}$obs is larger than one or for the $\mathrm{CL}_{\mathrm{S}}$ test if the corresponding CL_obs is smaller than 0.05. This statement is printed on the screen during the CheckmaTe run and can be found in the file result.txt at the top of the output directory. In the above example, it reads as follows:

\begin{tabular}{|lllllllll|}
\hline SR & S & dS_stat & dS_sys & dS_tot & S95_obs & S95_exp & $r^{\wedge} c_{-}$obs & $r^{`} c_{-} \exp$ \\
AL & 37.41 & 0.43 & 4.10 & 4.12 & 1341.20 & 1135.00 & 0.02 & 0.03 \\
AM & 5.37 & 0.15 & 0.56 & 0.58 & 51.30 & 42.70 & 0.08 & 0.10 \\
BM & 7.33 & 0.18 & 0.76 & 0.78 & 14.90 & 17.00 & 0.39 & 0.34 \\
BT & 0.84 & 0.05 & 0.10 & 0.11 & 6.70 & 5.80 & 0.09 & 0.11 \\
CM & 18.22 & 0.30 & 2.04 & 2.07 & 81.20 & 72.90 & 0.17 & 0.19 \\
CT & 2.32 & 0.08 & 0.27 & 0.28 & 2.40 & 3.30 & 0.74 & 0.54 \\
D & 12.15 & 0.24 & 1.29 & 1.32 & 15.50 & 13.60 & 0.62 & 0.70 \\
EL & 21.56 & 0.33 & 2.38 & 2.41 & 92.40 & 57.30 & 0.18 & 0.29 \\
EM & 16.08 & 0.29 & 1.79 & 1.81 & 28.60 & 21.40 & 0.44 & 0.59 \\
ET & 8.10 & 0.20 & 0.89 & 0.91 & 8.30 & 6.50 & 0.76 & 0.97 \\
\hline
\end{tabular}

FIG. 5: Example result of the conservative $r$ test defined in Equation (1), written into

evaluation/atlas_conf_2013_047_r_limits.txt. The observed and expected upper 95\% confidence limits on the number of signal events is taken from the respective experimental source. 


\begin{tabular}{|llllllllllll|}
\hline SR & S & dS_stat & dS_sys & dS_tot & B & dB & 0 & CL_obs & dCL_obs & CL_exp & dCL_exp \\
AL & 37.41 & 0.43 & 4.10 & 4.12 & 4700.00 & 500.00 & 5333.00 & 0.9754 & 0.0147 & 0.9204 & 0.0224 \\
AM & 5.37 & 0.15 & 0.56 & 0.58 & 122.00 & 18.00 & 135.00 & 0.9269 & 0.0137 & 0.8307 & 0.0210 \\
BM & 7.33 & 0.18 & 0.76 & 0.78 & 33.00 & 7.00 & 29.00 & 0.3453 & 0.0137 & 0.4566 & 0.0125 \\
BT & 0.84 & 0.05 & 0.10 & 0.11 & 2.40 & 1.40 & 4.00 & 0.8518 & 0.0095 & 0.6890 & 0.0158 \\
CM & 18.22 & 0.30 & 2.04 & 2.07 & 210.00 & 40.00 & 228.00 & 0.7395 & 0.0136 & 0.6691 & 0.0171 \\
CT & 2.32 & 0.08 & 0.27 & 0.28 & 1.60 & 1.40 & 0.00 & 0.1063 & 0.0077 & 0.2225 & 0.0082 \\
D & 12.15 & 0.24 & 1.29 & 1.32 & 15.00 & 5.00 & 18.00 & 0.1590 & 0.0049 & 0.0824 & 0.0041 \\
EL & 21.56 & 0.33 & 2.38 & 2.41 & 113.00 & 21.00 & 166.00 & 0.7574 & 0.0072 & 0.3704 & 0.0109 \\
EM & 16.08 & 0.29 & 1.79 & 1.81 & 30.00 & 8.00 & 41.00 & 0.3927 & 0.0064 & 0.1296 & 0.0055 \\
ET & 8.10 & 0.20 & 0.89 & 0.91 & 2.90 & 1.80 & 5.00 & 0.0609 & 0.0027 & 0.0077 & 0.0013 \\
\hline
\end{tabular}

FIG. 6: Example output of the $\mathrm{CL}_{\mathrm{S}}$ test written into evaluation/atlas_conf_2013_047_cls_limits.txt. B (expected background), $\mathrm{dB}$ (error on the expected background) and $\mathrm{O}$ (observed events) are taken from the experimental collaborations.

Test: Calculation of CLs from determined signal

Result: Allowed

Result for CLs: cls_min $=0.0695756126718$

Result for $r: r \max =0.757777916596$

SR: atlas_conf_2013_047 - ET

It should be noted that the $r$-limit is usually weaker than the $\mathrm{CL}_{\mathrm{S}}$ result, as the first uses the total uncertainty on the signal in a more conservative manner than the latter. It is therefore not impossible that $\mathrm{CL}_{\mathrm{S}} \lesssim 0.05$ and $r \lesssim 1$, i.e. $\mathrm{CL}_{\mathrm{S}}$ excludes but $r$ allows the input model. In those cases, the model will still be considered excluded, since if $\mathrm{CL}_{\mathrm{S}}$ is evaluated, it will exclusively be used to determine the final exclusion statement.

\section{B. Delphes Tunings}

Each of the given event files will be processed with version 3.0.10 of the fast multipurpose detector simulation Delphes [1. This tool includes the experimental resolutions and efficiencies of the two LHC multipurpose detectors, ATLAS and CMS, by parametrising a large list of detector effects (see Figure 1 or details in Ref. 1]). However exhaustive this list seems, many analyses CheckMATE uses need extra functionalities, which lie beyond the published version of Delphes. We have added these extra functionalities as well as re-parametrised many of the pre-existing functions of Delphes in order to better model the LHC detectors. These changes were motivated by detailed comparisons to the experimental results of individual simulation components which showed that the standard Delphes configuration needed to be improved. In the remainder of this section we qualitatively describe these changes in detail. Quantitative results are listed in Appendix C

\section{Improved Description of Lepton Reconstruction}

In order to properly estimate the measurement of leptons inside a detector, there are two main effects which have to be taken into account:

1. Inaccuracies in both the tracker and the calorimeter or muon spectrometer lead to an uncertainty in the kinematical properties of the lepton candidate. These can be estimated by applying a Gaussian smearing on every candidate, depending on its energy and position in the detector.

2. Algorithms to reconstruct electrons inside the calorimeter and to identify muons by associating tracks to hits in the muon chamber might fail. Hence a given truth lepton should only appear in the list of reconstructed lepton objects with a probability $\epsilon_{\ell}<1$ describing the reconstruction/identification efficiency.

There exist sophisticated experimental studies which provide quantitative statements for these effects, e.g. in the form of probability functions. These can be used in detector simulations like Delphes to reproduce the experimental effects

\begin{tabular}{|lllllllll|}
\hline analysis & bestSR & $r_{-o b s^{` c}}$ & $r_{-} \exp ^{` c}$ & CLs_obs & dCLs_obs & CLs_exp & dCLs_exp & {$[\ldots]$} \\
atlas_conf_2013_047 & ET & 0.76 & 0.97 & 0.0609 & 0.0027 & 0.0077 & 0.0013 & {$[\ldots]$} \\
\hline
\end{tabular}

FIG. 7: Example output written into evaluation/best_signal_regions.txt. For each analysis that has been considered in the given run, this file sums up the results of the signal region with the largest expected sensitivity. 
without having to reproduce all the internal details. We provide references to all the studies we used in this section and Appendix C.

Delphes uses text files, so-called detector-cards, which provide all necessary functions for both ATLAS and CMS, such that the users do not need to parametrise these effects themselves but can use the simulation straight away. However, on further investigation it turns out that the implemented standard functions are rather simplified versions of the usually complex distributions the experiments measure. Depending on the kinematics of the model and the considered analysis, the results from the simulation can deviate significantly from the experimental measurement.

Checkmate has therefore replaced most of these functions, using publicly available data from the experiment: 8 These include:

- Detailed $p_{\mathrm{T}}$ and $\eta$ dependent reconstruction and identification efficiency functions for 'medium' and 'tight' electrons 9 [4, 45].

- Muon reconstruction efficiency with respect to the type of detector that will measure the candidate according to its position, using a $\phi-\eta$ map of the muon spectrometer [46].

- Gaussian smearing of the momenta of the electron and muon candidates with $E_{T^{-}}$and $\eta$-dependent width 44, 46, 48.

Improved Description of Jet Tagging

Jets that originate from $b$-quarks or hadronically decaying $\tau$-leptons can in some cases be distinguished from other hadronic jets, e.g. by measuring displaced vertices or reconstructing a distinctive track signature. Sophisticated algorithms are used by the experimental collaborations on every reconstructed jet object and tag them as a $b$-quark or a $\tau$ if they pass certain criteria. These algorithms usually have various working points depending on how pure a sample is demanded and what background rejection is required. Similar to the lepton efficiencies, Delphes already provides rough efficiency functions for these tagging algorithms, which have been replaced by more detailed functions in Checkmate:

- $p_{\mathrm{T}}$ dependent $b$-quark identification efficiency and mis-tagging probability for jets with and without a $c$-quark component $49[52$.

- $p_{\mathrm{T}}$ dependent $\tau$ tagging efficiencies for signal and background, distinguishing between 1-track and 3-track candidates and deducing the candidate's charge from the combined charge of the track particles [53].

Flag Members for Efficiency, Isolation and Tagging

Delphes provides an isolation module, which checks the calorimeter and/or tracking environment around a particular candidate and removes that candidate if there is too much activity in its vicinity. This functionality is sufficient if only one analysis is being performed with Delphes and the analysis in question only requires a single isolation condition. However, many analyses require at least two different isolation conditions per final state object. Examples are many of the exclusive ATLAS lepton studies that use loosely isolated leptons in veto conditions but require tightly isolated leptons for signal regions.

The same problem appears for efficiencies, which remove objects with a particular $E_{T^{-}}$and $\eta$-dependent probability to parametrise losses during reconstruction and identification. These efficiencies depend on the precise experimental definition of the object in question. Many analyses use a loose definition — corresponding to a relatively large efficiency - to perform event vetoes or overlap removals, and then use stronger requirements with smaller efficiency for the actual signal objects. For jet tagging, i.e. for labelling a jet as having most likely originated from a $b$ quark or a hadronically decaying $\tau$ lepton, the original version of Delphes only provides binary member variables to state whether a particular tagging test succeeded or not. Therefore problems arise as soon as one needs to store results of multiple tagging algorithms, e.g. with different working points for the signal efficiency.

For these purposes, charged lepton ${ }^{10}$, photon and jet objects have been extended by one additional member, called flag, for each of the above mentioned checks, with the tagging being used exclusively for jets: If the $n$th flag condition

\footnotetext{
${ }^{8}$ For now, these improvements have only been implemented in Checkmate for the ATLAS experiment. They are planned for CMS at a later point.

9 'loose' electrons are considered in a special manner, see Appendix C

10 Throughout this article, the word 'lepton' excludes $\tau$ leptons, which are reconstructed as jets with a $\tau$ tag.
} 
succeeds, the member's flag value is increased by $2^{n}$. The final flag value of a candidate therefore represents, using binary decomposition, which tests have been passed or failed, and as such allows different test outcomes to be checked independently. The current implementation allows for the check of at most 32 independent conditions per flag.

\section{Detector Simulation Settings}

As described above, Delphes has been modified to be able to produce all necessary information needed by the various analyses. To do so, the following information is required for each analysis:

- The experiment (ATLAS or CMS) the analysis corresponds to,

- the $\mathrm{dR}$ cone parameter and $p_{\mathrm{T}}^{\min }$ for the FastJet program (all current analyses in CheckMATE use the anti-kt jet clustering algorithm [4]),

- potentially, the dR parameter of a second type of jet,

- the working signal efficiencies of all required b-/ $\tau$-tagging algorithms and

- which isolation criteria for electrons/muons/photons have to be tested, including

- the size $\Delta R$ of the cone around the candidate used to define the isolation criterion,

- whether tracks or calorimeter entries should be used for the isolation ${ }^{11}$

- the minimum $p_{\mathrm{T}}$ of objects within the $\Delta R$ cone to be taken into account and

- the maximum $\sum_{\Delta R} p_{\mathrm{T}}$ (absolute or relative to $p_{\mathrm{T}}^{\text {cand }}$ ) that is allowed within the cone of a candidate.

Merge of Settings

Many of the aforementioned settings are identical for different analyses, e.g. signal electrons with the same identification efficiency, jet tags corresponding to common working points or identical isolation conditions for final state objects. Before each run, CheckmATE collects the individual requirements of each chosen analysis, checks for common settings and merges them to a detector card with the smallest number of necessary modules. This both saves computing time and significantly reduces the size of the Delphes result files.

\section{ANALYSES}

Checkmate provides a variety of different analyses against which the users can test their models. In this section we give an overview of what defines a particular analysis. See Appendix B for details on where this information can be found within Checkmate and what users who would like to create their own analysis need to provide.

\section{A. Analysis Code}

The analysis part of Checkmate is based on the program package Root which takes as input the Rootfile output of the modified Delphes detector simulation. The final output from any analysis contains the numbers of events that pass each recorded cut and the final number of events in all the signal regions of interest, as can be seen for an example in Figure 3. All counters for these cutflows and the various signal regions are defined in the initialization process. In addition, kinematical variables relevant for the analysis can be given here.

The analysis begins with the definition of reconstructed final state objects. Potential isolation and tagging conditions must be defined in the relevant Delphes detector card, see Footnote 10 . If relevant for the analysis, trigger cuts on the events have to be imposed, such as cuts on the transverse momentum of leptons, jets or transverse missing momentum. Many of the experimental analyses are based on triggers that are not $100 \%$ efficient for all signal regions. Unfortunately, as of now, the experimental collaborations do not provide detailed efficiency maps for the employed

11 The distance parameter $\Delta R$ is defined as $\sqrt{\Delta \eta^{2}+\Delta \phi^{2}}$, where $\Delta \eta$ denotes the pseudorapidity difference and $\Delta \phi$ the relative angle projected onto the plane perpendicular to the beam axis. 
triggers. In the absence of this data, Checkmate analyses uses tuned triggers according to the supplied cutflows and parameter scans to match the experimental result as closely as possible.

Many analyses apply various cleaning cuts to remove beam and/or cosmic background events. However, since Checkmate is not running with real experimental data, no event cleaning needs to take place. In order to account for the loss of experimental luminosity due to these effects however, the reduction in the number of events used in the experimental analysis is applied as a flat efficiency factor. The reduction depends on the particular analysis but is never more than a few percent.

The analysis code tries to replicate as closely as possible the analysis structure of the corresponding experimental study. Cuts are placed on various final states and kinematical variables ${ }^{12}$ and corresponding cutflow counters keep track of the number of events that survive each cut.

The analyses in Checkmate all follow the philosophy that whenever a cutflow table is presented in the original study, the analysis follows the cutflow exactly. Following the corresponding cutflows has the consequence that the analyses are not optimised for computing time performance. However, we believe that this is a sacrifice worth making since the running time of the analysis is usually small in comparison to the event generation steps and detector simulation. The big advantage of our approach is that the same analysis code that is used in validation is also used for setting limits. In addition, users can check the particular cutflow themselves. At the end of the analysis, events are counted in each of the signal regions used to set limits. For a detailed overview on how to extend Checkmate with your own analysis, please see Appendix B.

\section{B. Reference Data}

The reference data that Checkmate uses to set limits on models all come from the corresponding experimental analysis. Since different analyses provide different levels of detail regarding errors, backgrounds and experimental limits, the format is flexible, but a certain minimum set of information is required.

As already described in Section IB. Checkmate can set limits on the model that is being tested in two different ways. One method is to compare the number of events in the most sensitive signal region with the $95 \%$ exclusion given by the experiment, Equation (1). Note that, in order to determine which signal region should be used to set the limit, the expected limit for each signal region is also required ${ }^{13}$. The uncertainty of the expected limit, determined by the experimental collaboration, is also required and is split into positive and negative fields if this information is provided.

The second method is to calculate the likelihood for the model using the $\mathrm{CL}_{\mathrm{S}}$ prescription, which requires the following information:

- The number of background events expected for a particular signal region along with the associated error. If given, the error is split into statistical and systematic components, each of which have positive and negative fields.

- The number of observed events in the signal region.

\section{Currently Validated Analyses}

The following analyses have been implemented in Checkmate and the results have been cross-checked against the published results. The current analyses were chosen to give a broad overview of the current ATLAS searches for final states containing a significant amount of missing transverse momentum, plus various combinations of jets, leptons and b-tags. In particular, we included the most sensitive ATLAS New Physics searches for final states containing an invisible particle.

In the following section we briefly discuss the corresponding signatures and example cases to which the analysis has been optimised.

12 More advanced observables such as the stransverse mass are included by integrating the Oxbridge Kinetics Library into Checkmate 6. 7.

13 For experimental analyses that do not contain this information, the $95 \% \mathrm{CL}_{\mathrm{S}}$ has been pre-calculated by CheckMATE so that this method is always available. 
atlas_conf_2012_104: 1 lepton $+\geq 4$ jets $+\mathbb{E}_{T}$, 27]

The one lepton search requires one isolated electron or muon with at least four jets and large missing transverse momentum in the final state. The study has two independent signal regions corresponding to an electron as well as a muon channel.

The study focuses on the pair production of new strongly interacting particles which then decay primarily into jets and a particle that evades detection, e.g. the Lightest Supersymmetric Particle (LSP) in SUSY. In addition at least one decay chain must contain a detected charged lepton. The search is motivated by the commonly studied mSUGRA (minimal supergravity) model where a left handed squark dominantly decays to a light chargino which subsequently produces a lepton pair and the LSP.

$$
\text { atlas_conf_2012_147: Monojet search }+\mathbb{E}_{T} \text {, [28] }
$$

The monojet search is optimised for a signal consisting of a single hard jet recoiling against missing energy. A second jet is allowed as long as the $\Delta \phi$ between the second jet and the missing $p_{T}$ is greater than 0.5 , but events with further jets are vetoed. Events containing reconstructed electrons, muons or hard photons are vetoed.

The signal regions have been optimised for a variety of signal models including graviton production in large extra dimension models [13, SUSY gravitino production in the context of gauge mediated SUSY breaking models 54 and a model independent search for dark matter [55]. In addition, if the model of new physics has a very compressed spectrum, this search can also become relevant [56, 57.

$$
\text { atlas_conf_2013_024: } 0 \text { lepton }+6 \text { (2 b-)jets }+\mathbb{E}_{T} \text { (All-hadronic stop search), [29] }
$$

The all-hadronic stop search requires at least 6 jets including $2 b$-jets to fully reconstruct two final state top quarks. In addition, a significant amount of $\mathbb{E}_{T}$ is required and a lepton-, including tau-, veto is applied. The search is targeted at SUSY top squark pair production where the top squark decays into a top quark and the LSP. However, the search can equally be applied to any model producing a pair of top quarks and significant missing energy in the final state.

$$
\text { atlas_conf_2013_035: } 3 \text { leptons }+\mathbb{E}_{T}, \text { [30] }
$$

The trilepton search considers final states containing three charged leptons (i.e. electrons or muons), with at least 2 being from a same-flavour opposite-sign (SFOS) pair, and missing transverse energy in the final state. It has six signal regions that are either enriched or depleted in $Z$ boson decays (the SFOS leptons lie within the $Z$ mass window). For each enriched and depleted $Z$ region, loose, medium and tight signal regions are defined. These correspond to tighter missing transverse momentum and transverse mass requirements.

The search focuses on the electroweak production of charginos and neutralinos with subsequent decays into final states with leptons and missing transverse momentum via slepton or electroweak gauge bosons.

$$
\text { atlas_conf_2013_047: 2-6 jets }+\mathbb{E}_{T} \text {, 31] }
$$

The multijet search is optimised for models with large production cross sections for heavy particles that decay to hard jets and a significant component of missing energy. For SUSY models this corresponds to squark and gluino production with direct decays into first or second generation quarks and the LSP. Signal regions with various numbers of final state jets and different proportions of missing energy are adapted to the different combinations of squark and gluino production.

$$
\text { atlas_conf_2013_049: } 2 \text { leptons }+\mathbb{E}_{T} \text {, [32] }
$$

The search for two opposite-sign leptons, missing transverse momentum and no jets in the final state focuses on electroweak production of charginos and sleptons. The study has five signal regions in total with two signal regions 
imposing a $Z$ veto and a low missing transverse momentum cut. The three remaining signal regions are sensitive to on-shell $W$ bosons and require stricter cuts on the transverse momentum of the leptons.

Both signal regions with a $Z$ veto are optimised for direct slepton pair production or provide sensitivity to sleptons from chargino decays. The three remaining signal regions are adapted to direct chargino pair production with charginos subsequently decaying into a $\mathrm{W}$ boson and the LSP.

$$
\text { atlas_conf_2013_061: 0-1 leptons }+\geq 3 \text { b-jets }+\mathbb{E}_{T} \text {, [33] }
$$

The $3 b$-jets study concentrates on the pair production of a heavy coloured particle which then decays with at least $2 b$-jets in the final state, giving $4 b$-jets in total. The reason only $3 b$-jets are used is that the tagging algorithm has an efficiency of approximately $70 \%$, hence consequently requiring all $4 b$-jets to be tagged leads to a significant reduction in acceptance. In addition, a large $\mathbb{E}_{T}$ cut is used in all signal regions.

The primary motivation for the search is to find gluino pair production followed by the decay into the LSP and either a pair of bottom quarks or top quarks. Another possible application is the production of Higgs bosons in decay chains, since the dominant decay mode of the Higgs is into $2 b$-jets.

$$
\text { atlas_conf_2013_089: } 2 \text { leptons }+ \text { jets }+\mathbb{E}_{T}(\text { razor }),[35]
$$

This 2-leptons + jets study focuses on the strong production of new states followed by decays that include leptons. A specific emphasis is placed on looking for soft leptons and the study allows for both electrons and muons to have transverse momenta as low as $10 \mathrm{GeV}$. To gain a better discrimination between the signal and SM backgrounds, the 'Razor' [58] variables are used. These variables exploit the symmetry that can be expected in the pair production and decay of new states with sizable missing momentum.

cms_1303_2985: At least 2 jets + b jet multiplicity $+\mathbb{E}_{T}\left(\alpha_{T}\right), 37$

This all hadronic search uses the $\alpha_{T}$ variable to search for strongly produced high mass states that decay to hard jets and a significant component of missing energy. The strength of the $\alpha_{T}$ variable is that it is robust against jet energy mis-measurements of $\mathbb{E}_{T}$ and consequently can search for new physics at lower jet $p_{\mathrm{T}}$ than more traditional 'jet $+\mathbb{E}_{T}$ searches'. There are also signal regions containing different $b$-jet multiplicities to search for bottom and top quark partners.

We remind the reader that the CMS detector is currently only modelled by the default Delphes settings for final states and has not been tuned to the latest experimental data. However, in order to simulate realistic $b$-tagging functionality, the ATLAS performance efficiency is used.

\section{PERFORMANCE STUDIES}

\section{A. Validation}

As stated in Section IIIB, the default detector settings of Delphes have been significantly improved to match the latest ATLAS performance. In particular, all final state objects have been matched within the experimental uncertainties to the latest data available and the detailed results are given in Appendix C. These tunings have been validated by comparing with some ATLAS analyses of Standard Model processes. As an example we show the reconstruction of SM $Z$ production ${ }^{14}$ versus ATLAS data for both electrons and muons in Figure 8 More detailed results are given in Appendix C

Before any analysis is accepted into the the latest version of CheckMATE it must be validated against the various data given in the published result. Whenever cutflows are given, these are checked at each step to make sure that all experimental cuts perform as expected, c.f. Appendix D The majority of the cutflows we have evaluated with Checkmate have an acceptance that is within $10 \%$ of the published value for the signal regions. If this accuracy is

14 Note that this analysis has been performed at $\sqrt{s}=7 \mathrm{TeV}$ and the efficiencies Checkmate used for the results shown in Figure 8 correspond to the detector performance during this phase. However, recently ATLAS improved their electron reconstruction and identification algorithms with better overall efficiency factors. Checkmate uses these updated results for its public version. 


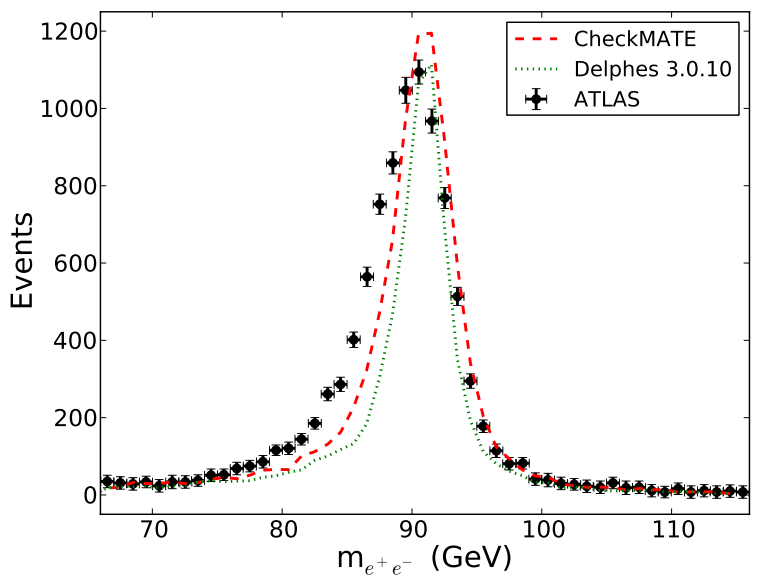

(a) Invariant mass of dielectron pairs in $Z \rightarrow e^{+} e^{-}$.

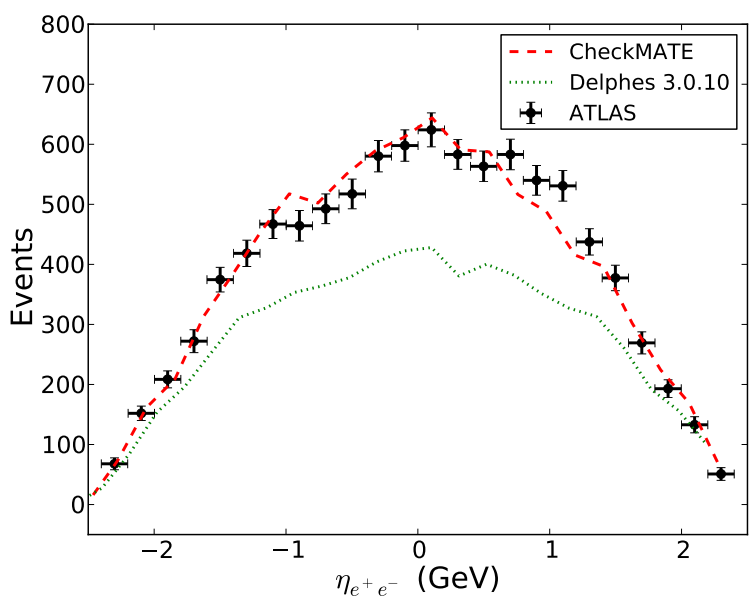

(c) Total pseudorapidity of dielectron pairs in $Z \rightarrow e^{+} e^{-}$.

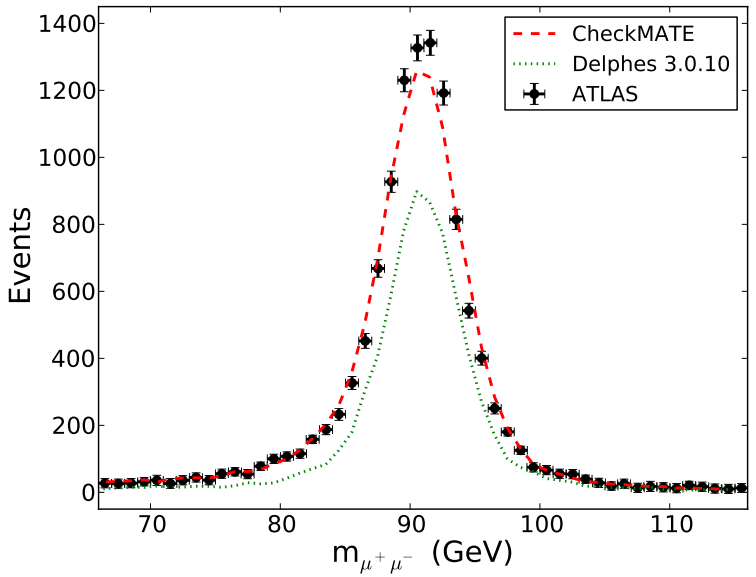

(b) Invariant mass of dimuon pairs in $Z \rightarrow \mu^{+} \mu^{-}$.

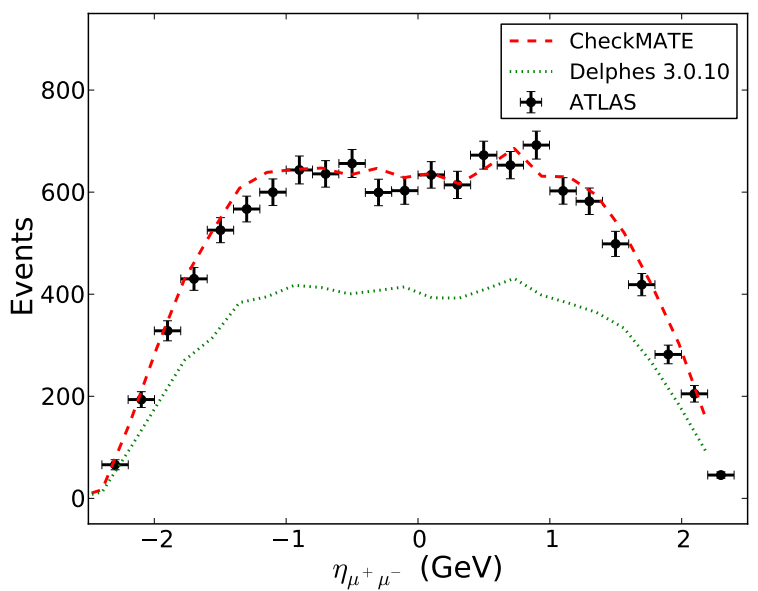

(d) Total pseudorapidity of dimuon pairs in $Z \rightarrow \mu^{+} \mu^{-}$.

FIG. 8: Comparison of results in dilepton channels of ATLAS $Z$-boson searches [59]. We use the same set of input events, produced with Pythia8, and process them separately with Delphes 3.0.10 using the standard ATLAS detector card and the Delphes version implemented in CheckmATE using the described detector tunings. The results of both Delphes runs are then analysed by identical analysis codes. Discrepancies in the dielectron width are most likely caused by problems in the full consideration of ISR/FSR effects within the simulation.

not achieved, the analysis has been checked thoroughly to ensure no bugs remain and possible (or confirmed) reasons for the discrepancy are clearly stated, see Appendix D

Many of the experimental analyses give exclusion curves for some popular (or simplified) models. Whenever possible these have been replicated by Checkmate (Appendix D) and in general the results lie within the $1 \sigma$ theoretical uncertainty predicted by the model. Again, in the rare occasions that the required accuracy has not been met, possible reasons for the discrepancy are given. An example parameter scan is shown in Figure 9 for the commonly studied SUSY model mSUGRA for the ATLAS jets and $\mathbb{E}_{T}$ search (atlas_conf_2013_047) that shows agreement within the $1 \sigma$ theoretical uncertainty for almost the whole plane. 

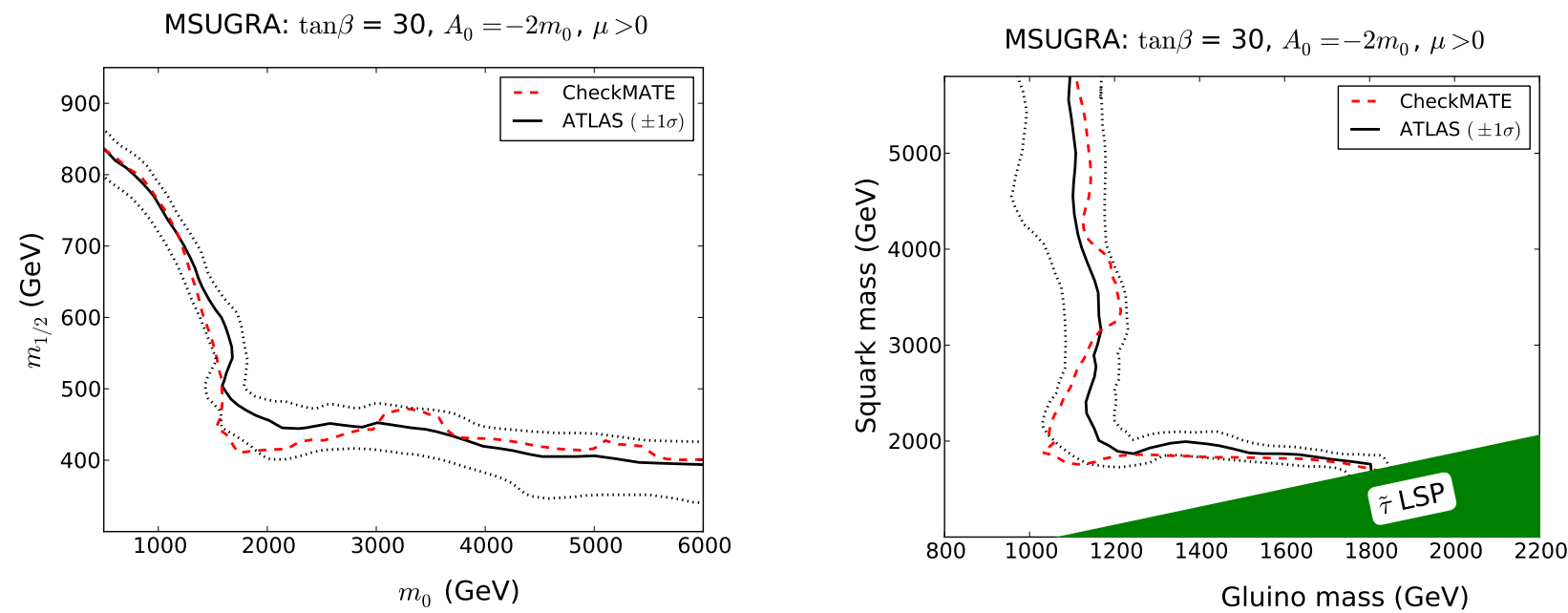

FIG. 9: Exclusion curve for the CMSSM (mSUGRA) model for analysis atlas_conf_2013_047, in the $\left(m_{0}, m_{1 / 2}\right)$ (left) and $\left(m_{\tilde{q}}, m_{\tilde{g}}\right)$ (right) planes.

\section{B. Computing Performance}

As an example to show the relative computing time of CheckMATE we consider an estimate from the ATLAS trilepton study (atlas_conf_2013_049). The generation of $1000 \tilde{\chi}_{1}^{+} \tilde{\chi}_{1}^{-}$events in the HepMC format takes about 121 seconds of CPU timf ${ }^{15}$ with Herwig++ 2.6.3. The Delphes 3.0.10 detector simulation with the relevant detector cards from Checkmate requires 11 seconds of CPU time. Once the detector simulation is finished, running the ATLAS trilepton analysis only takes about 1 second for all five signal regions. Thus the running time of the analysis is negligible compared to the event generation and detector simulation. This justifies our choice to closely match the cutflows of the experiments and thus add reliability and not to optimise the analyses for computing time performance.

\section{OUTLOOK}

We would like to emphasise that while Checkmate is fully functional, it remains a work in progress. We will improve and extend the program over time.

At the moment, we have concentrated on searches for supersymmetry from ATLAS. However, the various supersymmetric analyses already cover multiple final state configurations and thus should be sensitive to many nonsupersymmetric BSM physics scenarios containing invisible particles. Nevertheless, we want to implement as many of the new exotic searches from ATLAS and CMS as possible. We hope that the user will have access to an even larger selection of analyses in the near future.

Furthermore, we have decided to implement the results of the conferences notes rather than taking analyses from journal publications. Our decision is justified due to the fact that the majority of published results have not so far taken into account the $8 \mathrm{TeV}$ data and we are convinced that the implementation of studies with the best kinematical reach is the better alternative. As soon as the published results become available, we will update our studies.

Checkmate already provides a large number of validated analyses and many more only require final validation. Moreover, the structure of the program allows for easy implementation of additional analyses by any user. If an analysis has been programmed we would welcome the opportunity to include the study in the official CheckMATE library. After the new analysis has been validated, it would then be made available to the whole user community.

As discussed, we have significantly improved the detector simulation performance of Delphes. However, we would like to implement even better detector tunings. In particular, when ATLAS and CMS publish updated detector performances, it is planned that we will revise the detector tunings to take advantage of the new information 16 .

15 The CPU time performance has been tested on an Intel Core 2, Quad CPU with $2.4 \mathrm{GHz}$ and 8 GB RAM.

16 We would like to take this opportunity to plead with the collaborations to provide more detail in regards to detector performance. In particular, final state object maps for both identification and reconstruction should be provided in terms of $p_{\mathrm{T}}$ and $\eta$ for all final state objects. 
In addition to improving the analysis base of Checkmate, we further plan to present applications (case studies) to investigate models that the collaborations have not yet been able to complete and which we hope illustrate the power of CheckmATE. When these studies are available we will publish programs that enable the user to repeat these studies and easily run new parameter scans themselves.

Further into the future we plan to significantly extend the functionality of Checkmate to aid usability. Firstly we plan to include a Monte-Carlo generator into the program so that users no longer have to generate event files themselves. Along with this innovation, will be a supersymmetry SLHA file reader requiring only a supersymmetric spectrum as input. For models other than supersymmetry we plan a close integration with FeynRules [60] and SARAH [61] so that the user can begin their CheckMATE investigations armed only with a Lagrangian.

\section{SUMMARY}

We have introduced the program CheckMATE, which is a tool to automatically check the compatibility of BSM models with LHC data. The program takes input in the form of Monte Carlo event files and cross-sections. These event files are given to a detector simulation and the result passed to implementations of LHC analyses. The outcome of the analyses is then compared to LHC results to see if the given model can be excluded.

The program has been designed for easy installation and operation. In addition, the structure allows the user to include new experimental analyses in a straightforward manner. However, it must be emphasised that the current analysis status in CheckmaTe is in no way considered complete. It is foreseen that many additional LHC new physics searches will be included in future upgrades.

The code can be obtained from the Checkmate webpage

$$
\text { http://checkmate.hepforge.org }
$$

as well as the installation instructions, more detailed technical informations and frequently asked questions.

\section{ACKNOWLEDGEMENTS}

We would especially like to thank Andreas Hoecker for many detailed discussions regarding ATLAS analyses. In addition, we would like to thank Philip Bechtle, Jamie Boyd, Geraldine Conti, Carolina Deluca, Klaus Desch, Monica D'Onofrio, Frank Filthaut, Eva Halkiadakis, Emma Kuwertz, Zachary Marshall, Antoine Marzin, Alaettin Serhan Mete, Marija Vranjes Milosavljevic, Maurizio Pierini, Tina Potter, George Redlinger, Steven Worm and Frank Wuerthwein for helpful discussions regarding different analyses. On the theoretical side, Tim Stefaniak provided useful advice from his work with 'HiggsBounds/Signals'. The work has been supported by the BMBF grant 00160200. The work of M. Drees has been supported by the SFB TR33 "The Dark Universe" funded by the Deutsche Forschungsgemeinschaft (DFG). The work of J. S. Kim has been partially supported by the MICINN, Spain, under contract FPA2010- 17747; Consolider-Ingenio CPAN CSD2007-00042 and by the ARC Centre of Excellence for Particle Physics at the Terascale. J. S. Kim also thanks the Spanish MINECO Centro de excelencia Severo Ochoa Program under grant SEV-2012-0249. 


\section{Appendix A: Getting Started}

This section guides the user through the installation of CheckMATE and outlines how to check and potentially install updated versions of all the necessary prerequisites. The program has been tested and will run on both Linux and MacOS X ${ }^{17}$. An alternative online step-by-step tutorial can be found under http://checkmate.hepforge.org/ tutorial/start.php.

\section{Setting up Python}

Checkmate requires Python 2.7.X with $X \geq 3$ on your system. Most systems already come with a Python installation, which you can easily check by typing python $-\mathrm{V}$ and hitting 'enter' in a terminal. If Python is installed, it should start and immediately tell you the version number. If the installation is too old, too new ${ }^{18}$ or if there is no Python at all, please install it either manually from http://www.python.org/download/ or using the software management of your system.

\section{Setting up Root}

Checkmate uses Root for a variety of tasks, therefore it is necessary that every user has a fully working RooT installation available on their system. Furthermore, Checkmate uses some Root packages which are not installed automatically and which may need to be added. Due to the large size of the Root source code, we refrained from including it in our package and only provide a step-by-step tutorial for how to install it from scratch or add potentially missing obligatory packages to an existing installation. Running whereis root inside the terminal tells you if there already exists an installation on your system and determines how to continue with the installation:

Case 1: There is no Root installed yet: You must download the source and install Root from scratch. Please do not use the RoOT pre-compiled binaries but follow these instructions, since we encountered internal linking problems with the binary version of Root. Start by downloading the latest version from http://root.cern . ch/drupal/content/downloading-root with:

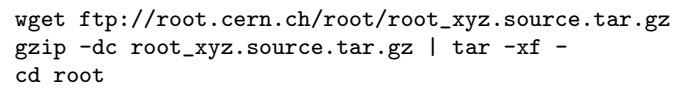

After downloading Root you can choose where you would like the installation located. If you don't want to do a system-wide installation in /usr/bin (e.g. because you do not have administrator rights), you have to use --prefix and --etcdir to declare the installation folder. Should you use a local Python installation (i.e. if the Python binary is not located in /usr/bin), you have to give the positions of both /include and /lib by adding --with-python-incdir=[path_to_python]/include/python2.7--with-python-libdir=[path_to_python]/lib with:

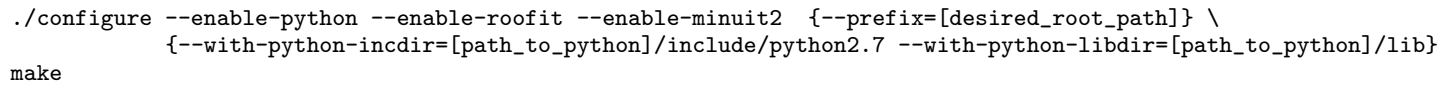

Root is large, so go and have a (big) cup coffee in the meanwhile. After a successful completion, please hit: make install

Case 2: There is already a Root installation on the system.: If you already have RooT on your system, let us first check if it includes all necessary packages. In the RoOT base directory, please run the following commands:

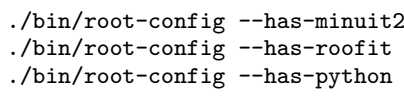

17 The installation on MacOS X systems require the Xcode Command Line Tools. Details for the installation can be found on http: //railsapps.github.io/xcode-command-line-tools.html and https://developer.apple.com/xcode/ Please note that wget is not pre-installed on MacOS X systems. However, it can be obtained from the MacPorts system https://www.macports.org or by downloading the respective files by hand.

18 Checkmate does not work with Python 3. 
If all three commands return 'yes', your Root installation includes everything CheckMATE needs and you can safely skip to the 'Setting up CheckmATE' section. If not, you have to recompile the code and add the missing packages. Follow the instructions mentioned for 'Case 1', but make sure that

- you download the same Root version the system has (you can use ./root-config --version to find out),

- you install into the same root-directory by choosing --prefix accordingly. Alternatively, you can always download and install a second root version locally according to step 1. Make sure that you set up Checkmate with this local RoOT version explicitly.

In case you are using the standalone Root binaries, beware that we sometimes encountered problems during the compilation of Checkmate. Should you find the same problems, i.e. that all the aforementioned checks were positive but still there are RoOT linking errors occurring, please consider using a proper installation from source code as explained above.

Setting up CheckMATE

Contrary to many other tools, Checkmate does not have a separate make install routine and will set itself up in the directory you put it. You should therefore begin the following procedure from within the folder you want Checkmate to be located.

Start by downloading the tarball either by hand or from within the terminal

wget http://www.hepforge.org/archive/checkmate/CheckMATE-Current.tar.gz

Extract the tarball and have a look at the CheckMATE folder:

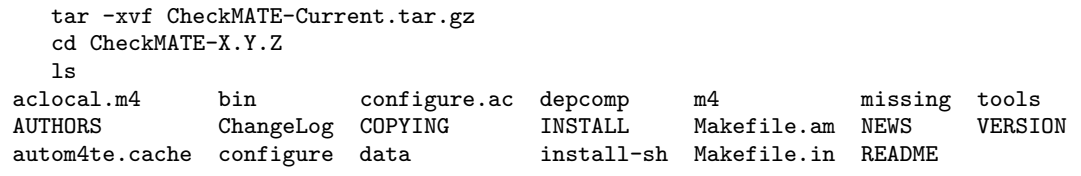

In order to run Checkmate we have to compile the code, connect the Python scripts to the Root libraries and create the binary. The configuration step will make sure that you followed the aforementioned sections to properly setup RooT and have a valid Python interpreter. The easiest way to proceed is to run ./configure and follow the instructions in case of an error. After successful configuration, type make ${ }^{19}$, which will create a Checkmate binary in bin/.

./configure $\{-$-with-rootsys=[path_to_root] $\}$ \{--with-python=[path_to_python]/bin/python $\}$

make

Note for experienced Root users: CheckMATE should work even if you haven't set up \$ROOTSYS (and similar) environmental variables since it takes all information from the --with-rootsys parameter. However in case of any unexpected errors, run thisroot. (c)sh inside the Root binary directory and retry.

To test the installation, run the test parameter file in the bin directory.

cd bin

./CheckMATE testparam.dat

The program will ask if the automatically chosen output directory is correct. After answering 'y' the program should run and finish with the statement that the input is allowed. If it doesn't, check whether your configuration and compilation produced any errors or warnings. Otherwise, you finished setting up CheckMATE and you can continue with Section II to learn how you can run the code.

19 Depending upon your system the make command can generate various warning messages. These can be safely ignored unless the final output is an error message (e.g. make: *** [all-recursive] Error 1) in which case the installation will have failed. If you are unable to understand the cause of the problem, please check the installation FAQ on the CheckmaTE website. 


\section{Appendix B: Adding Analyses}

Checkmate is not only meant to be simple to use and transparent. The philosophy of the program is that it is also supposed to be easy to extend with new ideas for analyses or users who need a particular analysis, which has not been implemented yet. In this section we give a brief introduction into the most important steps that are necessary to implement a new analysis.

\section{Using the Analysis Manager}

Checkmate comes with an Analysis Manager, i.e. a binary which asks the user for all the necessary details and takes care of the internal setup. In order to not confuse the normal user, this binary is not created automatically with the ordinary compilation of the code. However, it can easily be added by typing (after having already configured the code according to Appendix A

make AnalysisManager

This should create a second binary AnalysisManager within the bin/ folder. Running it will reveal three possible options:

- Show a list of all currently implemented analyses,

- add a new analysis/modify an existing analysis or

- remove an analysis.

Choosing the second option will guide the user through a large list of questions regarding the analysis. These gather information with respect to the general properties of the analysis like name and luminosity, the setup of the detector simulation (see Footnote 10 and all the considered signal regions with their respective experimental numbers for observation and background. These settings are written in human-readable form in data/ANALYSIS_var . $j$ and can be changed afterwards, by re-running the 'adding' step with the AnalysisManager 20 . Furthermore, the AnalysisManager creates ready-to-compile source and header files in tools/analysis/src and tools/analysis/include and adds them to the configuration files, such that they are compiled with the make routine.

\section{Analysis Code}

After the source files have been created, they have to be filled with the actual analysis code. For this, CheckMATE provides a general $\mathrm{C}++$ analysis framework using a globally defined base clas ${ }^{21}$ This class provides particle containers for all the different types of final state objects, like leptons or jets, alongside with various methods that are commonly needed in many analyses, like phase space reduction and overlap removals.

To keep this manual at a reasonable volume, we will only show a minimal example to illustrate the basic structure of the code in Figure 10. For more details on the full list of implemented procedures, see the CheckMATE webpage or the source code of the already implemented analyses. The shown example consists of a fictitious analysis with three signal regions and a two-step cutflow. It considers isolated electrons with $p_{\mathrm{T}}>20 \mathrm{GeV},|\eta|<2.5$, passing the 'medium' identification requirement and not being in the overlap region $1.37<|\eta|<1.52$. Furthermore it considers 'combined' muons and jets with different kinematical cuts. Jets that overlap with an isolated electron within $\Delta R_{e j}<0.2$ are discarded. The event is also vetoed if it does not contain exactly one lepton and at least two jets. One signal region demands the total scalar sum of all isolated jet energies to be larger than $150 \mathrm{GeV}$. The second and third signal region requires an isolated electron or muon, respectively, that has a small angular separation from the missing momentum vector.

We finish this section with some important information regarding analyses in general:

- Available particle containers are electronsLoose, electronsMedium, electronsTight, muonsCombinedPlus, muonsCombined, jets, jets2, photons, tracks and towers. jets2 only contains data if a second jet type has been defined in the creation of the analysis. towers and tracks are not needed by many analyses and are hence ignored in the standard analysis initialisation, meaning that the Root branch is not read.

20 Adding a new analysis will not only produce the described analysis variable file, but will also save the analysis information and the signal region numbers in separate auxiliary files, which are read by the respective Checkmate modules. Changing the analysis variable file will not update these auxiliary files automatically.

21 The concept of the code structure is based on the Rivet analysis framework 62]. 


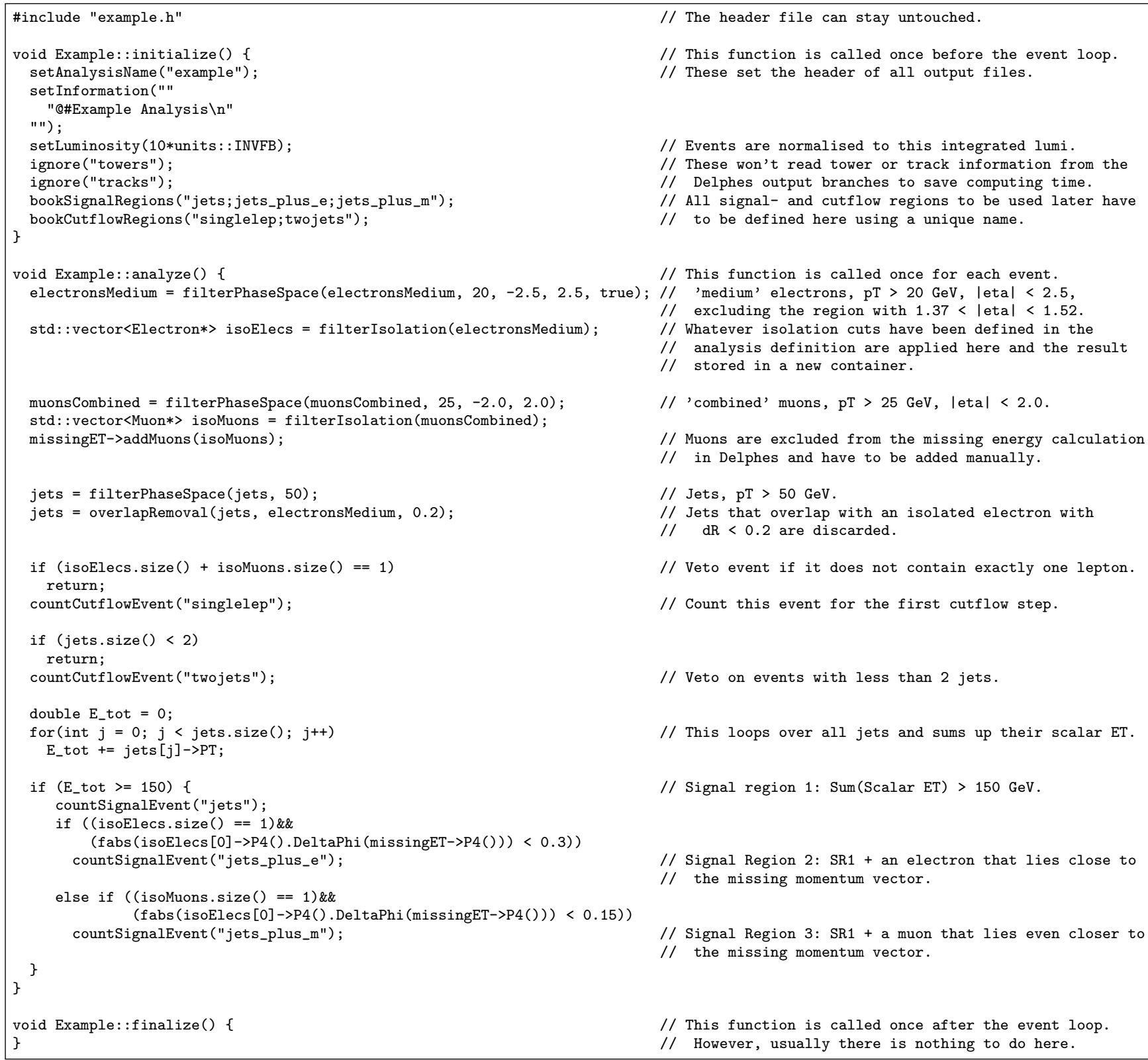

// The header file can stay untouched.

// This function is called once before the event loop. // These set the header of all output files.

// Events are normalised to this integrated lumi. // These won't read tower or track information from the // Delphes output branches to save computing time. // All signal- and cutflow regions to be used later have // to be defined here using a unique name.

// This function is called once for each event.

'medium' electrons, pT $>20 \mathrm{GeV}$, $\mid$ etal $<2.5$,

// excluding the region with $1.37<\mid$ etal $<1.52$.

// Whatever isolation cuts have been defined in the

// analysis definition are applied here and the result

// stored in a new container.

// 'combined' muons, $\mathrm{pT}>25 \mathrm{GeV}$, $\mid$ etal $<2.0$.

// Muons are excluded from the missing energy calculation $/ /$ in Delphes and have to be added manually.

// Jets, pT > $50 \mathrm{GeV}$

// Jets that overlap with an isolated electron with

// $\mathrm{dR}<0.2$ are discarded.

// Veto event if it does not contain exactly one lepton.

// Count this event for the first cutflow step.

// Veto on events with less than 2 jets.

// This loops over all jets and sums up their scalar ET.

// Signal region 1: Sum(Scalar ET) > $150 \mathrm{GeV}$.

// Signal Region 2: SR1 + an electron that lies close to $/ /$ the missing momentum vector.

// Signal Region 3: SR1 + a muon that lies even closer to $/ /$ the missing momentum vector.

// This function is called once after the event loop. // However, usually there is nothing to do here.

FIG. 10: Example code to illustrate the most basic features of a Checkmate analysis.

- These containers are of the standard $\mathrm{C}++$ vector type, string pointers to the respective particle objects.

- All vectors are sorted according to the objects' $p_{\mathrm{T}}$, with e.g. jets [0] having the highest transverse momentum.

- The final numbers for all signal-, control- and cutflow regions are automatically saved in the corresponding output file after the analysis run, listed in alphabetical order.

- Calling the P4() method returns the particle's 4-vector in the lab frame, using Root's TLorentzVector format which comes with a convenient list of applicable methods.

- The header file (example.h in this case) can stay untouched in most cases. However, to save computing time it might be reasonable to not define local variables in the analyze() method but instead define them as members of the analysis class within the header. 


\section{Appendix C: ATLAS Detector Tunings}

\section{Jets and Photons}

For both jets and photons, the standard Delphes parametrisations for reconstruction and identification have been used. Please see [1] for more details.

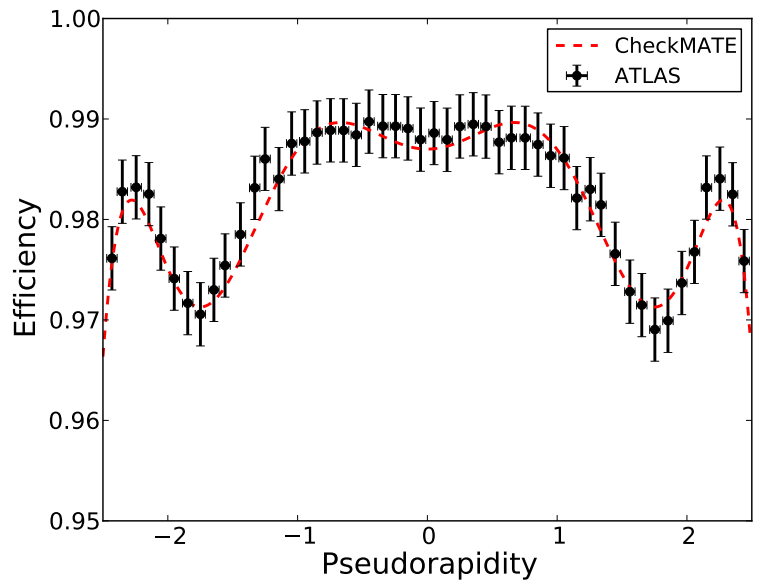

(a) Efficiency for the reconstruction of an electron object inside the calorimeter [45].

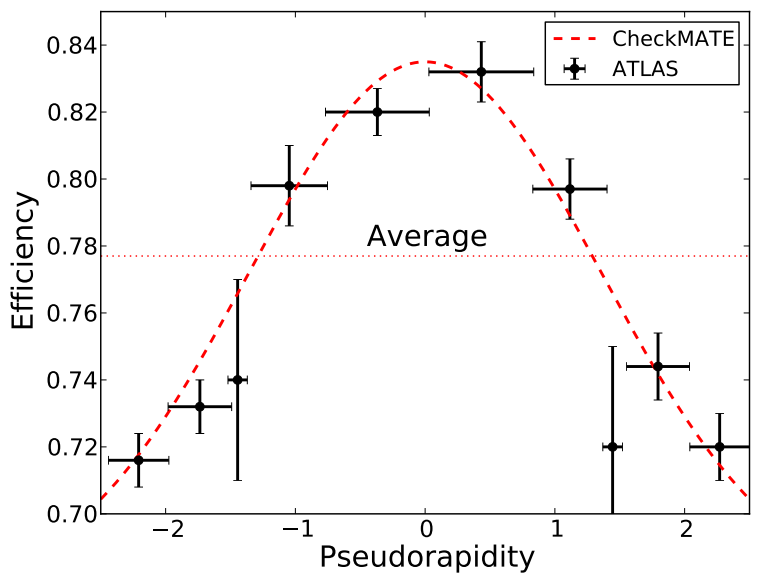

(c) Identification efficiency for 'tight' electrons as a function of the candidate's pseudorapidity [44].

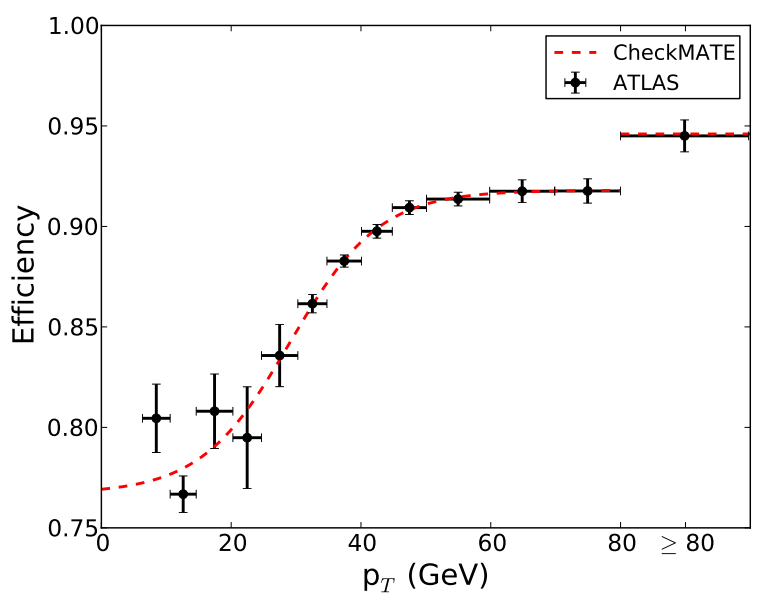

(b) Identification efficiency for 'medium' electrons [45].

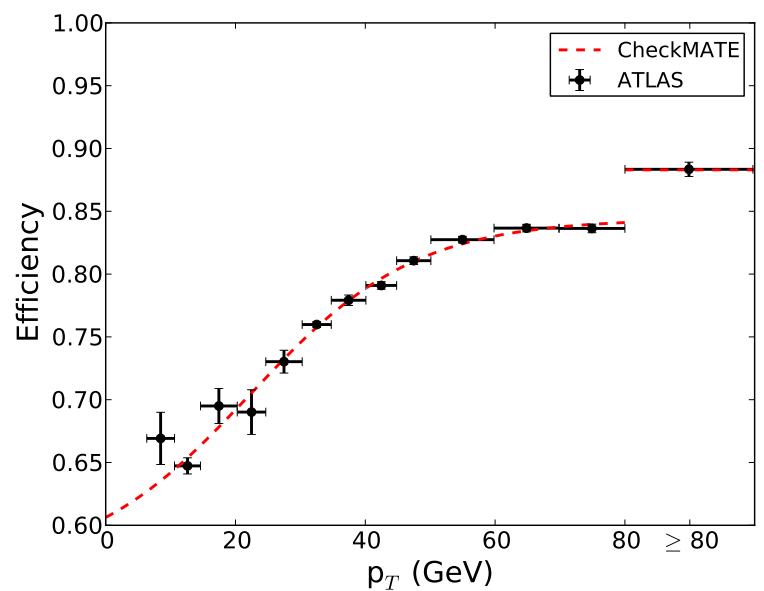

(d) Identification efficiency for 'tight' electrons as a function of the candidate's $p_{T}$ [45].

FIG. 11: Electron efficiency distributions used in CheckMATE. The functions that have been used to fit the data are given in Equations (C1) to C4). For the tight identification efficiency, CheckMATE uses the absolute value from the $p_{T}$ dependent efficiency shown in $\mathrm{d}$ ), and multiplies it with the $\eta$ dependent efficiency in c) normalised to an average value of 1 . 


\section{Electrons}

The efficiencies for electron objects to be reconstructed as such are combined from the individually measured reconstruction and identification efficiency, for which the latter distinguishes between two kinds of electrons: 'medium' and 'tight 22. The reconstruction efficiency does not show any significant dependence on the candidate's momentum but mostly on the pseudorapidity, as can be seen in Figure 11a. Conversely, the identification efficiency for medium electrons mostly depends on the transverse energy of the electron, as shown in Figure 11b, and insignificantly on its position. The total efficiency is given as the product of the two contributions and can be seen in a representative two-dimensional grid in Figure $12 \mathrm{a}$.

For 'tight' electrons, there exist measurements for both the pseudorapidity and the $p_{T}$ dependence of the identification efficiency individually, for which the corresponding other variable has been integrated over (see Figure 11c and Figure 11d). We normalise the pseudorapidity dependent function to an average value of unity and use as the overall identification efficiency the absolute efficiency with respect to $p_{T}$, multiplied by the renormalised efficiency with respect to pseudorapidity. Combining this with the reconstruction efficiency described before leads to the total efficiency distribution shown in Figure $12 \mathrm{~b}$.

The following functional behaviour for the efficiencies is used $\left(p_{T}\right.$ in $\left.\mathrm{GeV}\right)$ :

$$
\begin{aligned}
\epsilon_{\mathrm{rec}}(\eta) & =0.987+1.28 \times 10^{-2} \cdot \eta^{2}-1.76 \times 10^{-2} \cdot \eta^{4}+5.21 \times 10^{-3} \cdot \eta^{6}-4.49 \times 10^{-4} \cdot \eta^{8}, \\
\epsilon_{\mathrm{id}, \text { medium }}\left(p_{\mathrm{T}}\right) & = \begin{cases}0.767+\frac{0.151}{1+\mathrm{e}^{-0.145 \cdot\left(p_{\mathrm{T}}-29.1\right)}} & \text { if } p_{\mathrm{T}}<80 \\
0.946 & \text { if } p_{\mathrm{T}} \geq 80,\end{cases} \\
\epsilon_{\mathrm{id}, \text { tight }}\left(p_{\mathrm{T}}\right) & = \begin{cases}0.565+\frac{0.279}{1+\mathrm{e}^{-0.0786 \cdot\left(p_{\mathrm{T}}-22.3\right)}} & \text { if } p_{\mathrm{T}}<80 \\
0.883 & \text { if } p_{\mathrm{T}} \geq 80,\end{cases} \\
\epsilon_{\mathrm{id}, \text { tight }}^{\text {normalised }}(\eta) & =\frac{0.675+0.160 \cdot \exp \left(-\left(\frac{\eta}{1.92}\right)^{2}\right)}{0.777} .
\end{aligned}
$$

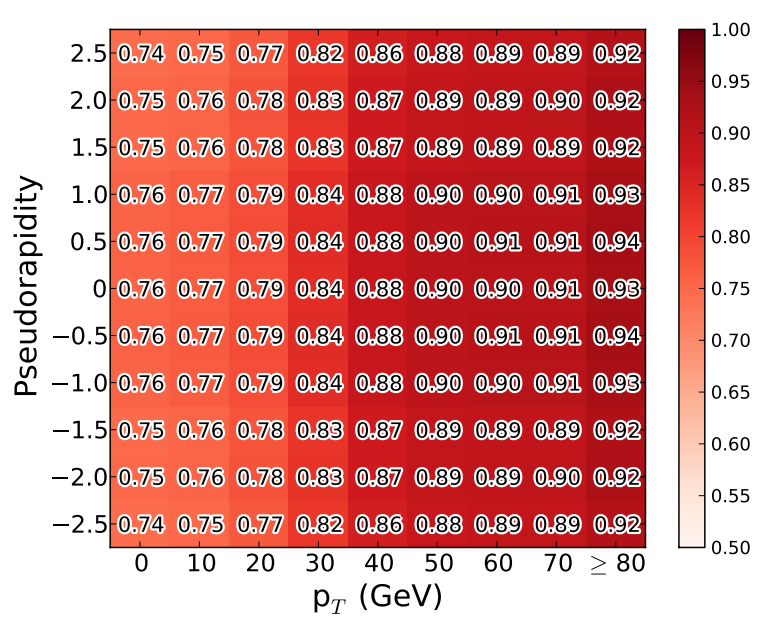

(a) Medium Electrons

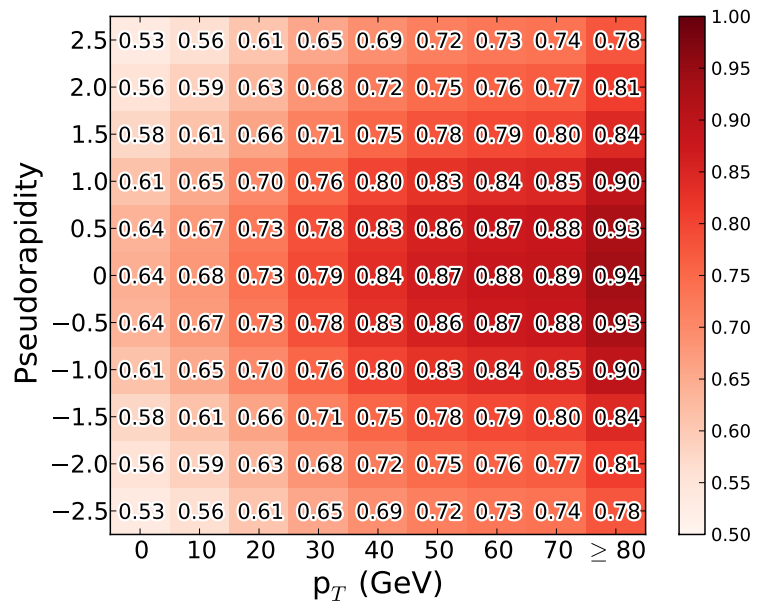

(b) Tight Electrons

FIG. 12: Total efficiency map with respect to pseudorapidity and energy. This discretised version is only meant for illustrative purposes; CheckMATE uses the full functional behaviour as shown in Equations (C1) to (C4).

\footnotetext{
22 The 'loose' electron efficiency for identifying a truth-level electron is estimated by implementing a weak calorimeter isolation that mimics the ATLAS reconstruction. We require that within a cone size $d R<0.2$ around the electron, at least $80 \%$ of energy deposition is due to the electron.
} 


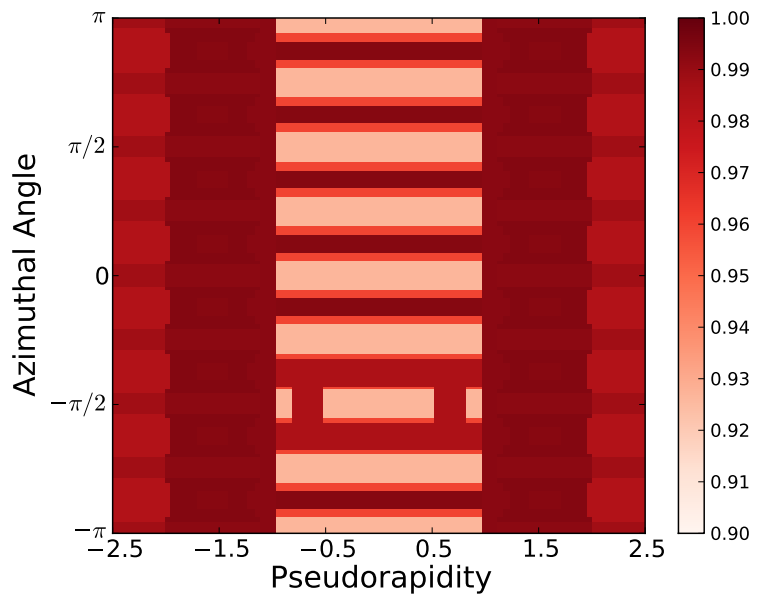

(a) Combined + Standalone Muons

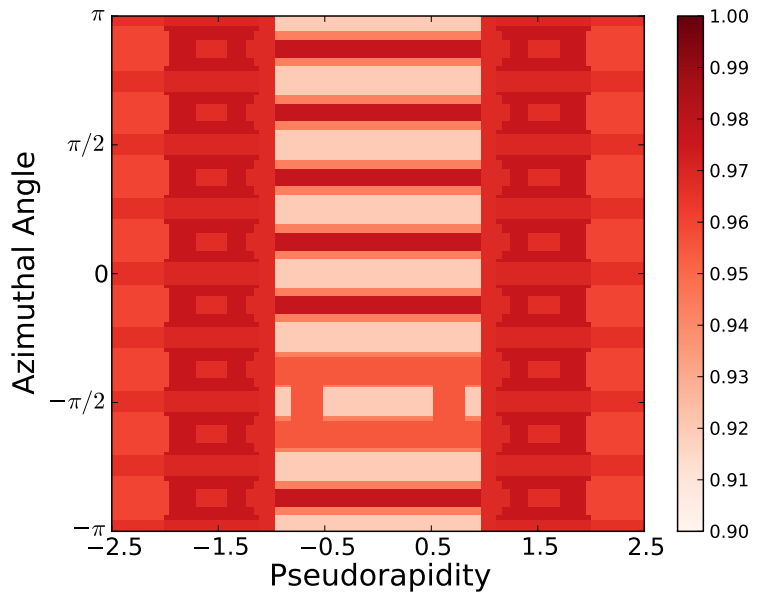

(b) Combined Muons

FIG. 13: Efficiencies for muon objects, depending on the position in the detector. Detector map and component-dependent efficiencies are taken from [46].

\section{Muons}

For muons, the main effect that has to be taken into account is the efficiency to reconstruct an object in the muon chambers and associate it to a track in the inner detector region. There exist two main quality criteria for this reconstruction, namely 'Combined' (requires both a track in the inner detector and muon chambers) and 'Standalone' (only requires a track in the muon chamber). The latter is usually used in combination with the first to maximise the muon efficiency and is hence called 'Combined+Standalone' (a standalone track is used when a combined track is not reconstructed).

The muon chambers in ATLAS consist of different components, which each have a different reconstruction probability, mainly caused by different types and quantities of material and the geometry inside the full detector. In Checkmate, we parametrised a detector-component map in the $\eta-\phi$ plane and associate a particular efficiency to each detector type. The resulting two-dimensional grie 23 is shown in Figure 13.

\section{B-Tagger}

The quality of algorithms that try to filter jets containing b-quarks from others is determined by two main quantities: The signal efficiency describes the probability to assign a tag to a jet that actually contains a b-quark, whereas the background efficiency is a measure for the relative amount of jets that are tagged even though they don't have any bottom quark content. Since the background efficiency is usually small, it is common to use the inverse value, called rejection, for illustrative purposes. Also, one usually distinguishes between rejections against jets with charm-content and other jets that only contain light quarks, as the first are harder to distinguish from the signal.

Since the rejection gets weaker with increasing signal efficiency, one has to find a balance between signal quantity and signal purity, which depends crucially on the details of the respective analysis. For this purpose, one uses the ROC (Receiver Operation Characteristic) curve that describes the relation between these two quantities. We show the ROC curves for light-jet rejection and c-jet rejection separately in Figure 14 and internally parametrise these as

\footnotetext{
${ }^{23}$ We refrain from showing the functional description of the full map inside the main text, as the fine and irregular segmentation leads to a hard-to-read $21 \times 53$ matrix. If further information is desired, the user should feel free to contact the authors.
} 


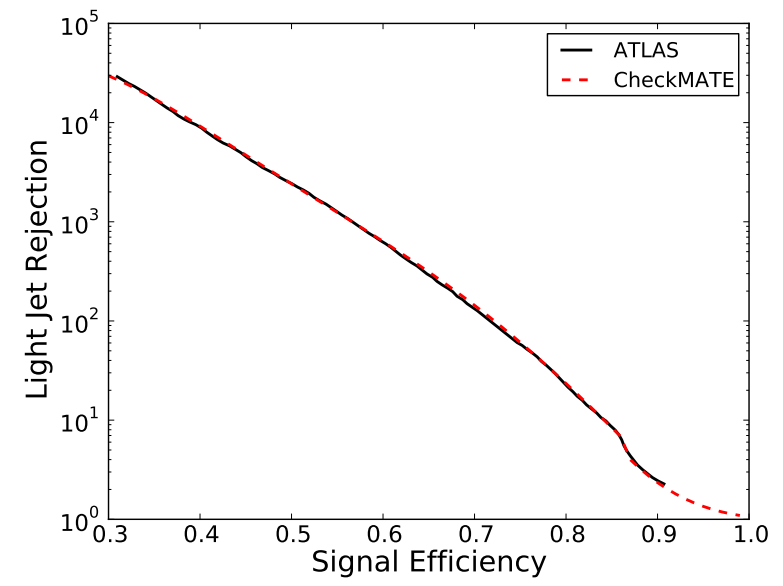

(a) Rejection curve for jets containing light quarks only.

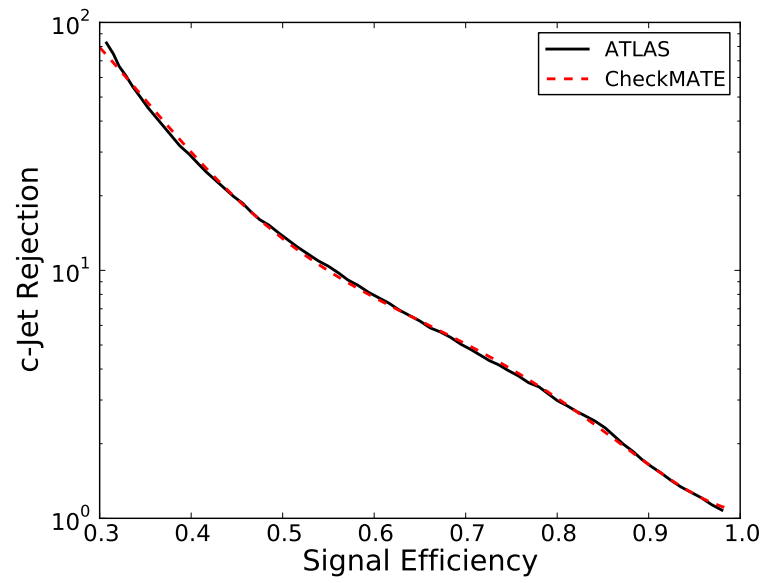

(b) Rejection curve for jets with charm content.

FIG. 14: Receiver Operation Characteristic curves for the dependence of the background rejection for jets with different quark contents on the chosen signal efficiency working point of the b-tagger. 63.

follows:

$$
\begin{aligned}
\log _{10}\left[\bar{r}_{\text {light }}\left(\bar{\epsilon}_{\mathrm{S}}\right)\right] & = \begin{cases}52.8 \cdot\left(\bar{\epsilon}_{\mathrm{S}}-4.045 \cdot \bar{\epsilon}_{\mathrm{S}}^{2}+7.17 \cdot \bar{\epsilon}_{\mathrm{S}}^{3}-6.14 \cdot \bar{\epsilon}_{\mathrm{S}}^{4}+2.01 \cdot \bar{\epsilon}_{\mathrm{S}}^{5}\right) & \text { if } \bar{\epsilon}_{\mathrm{S}}<0.87, \\
-75.4 \cdot\left(\bar{\epsilon}_{\mathrm{S}}-1.07\right)^{3} & \text { if } \bar{\epsilon}_{\mathrm{S}} \geq 0.87,\end{cases} \\
\log _{10}\left[\bar{r}_{\mathrm{c}}\left(\bar{\epsilon}_{\mathrm{S}}\right)\right] & =29.3 \cdot\left(\bar{\epsilon}_{\mathrm{S}}-4.572 \cdot \bar{\epsilon}_{\mathrm{S}}^{2}+8.496 \cdot \bar{\epsilon}_{\mathrm{S}}^{3}-7.253 \cdot \bar{\epsilon}_{\mathrm{S}}^{4}+2.33 \cdot \bar{\epsilon}_{\mathrm{S}}^{5}\right) .
\end{aligned}
$$

Given a particular working point on the ROC curve, i.e. a specific chosen signal efficiency $\bar{\epsilon}_{\mathrm{S}}$ and the corresponding background rejections $\bar{r}_{\text {light/c }}$, the actual tagging probabilities depend on the transverse momentum of the considered object. These have been measured individually for signal-, light-quark- and $D^{*}$ meson ${ }^{24}$ jets and we show the results in Figure 15.

For the signal efficiency, we use two different data sets as they have different sensitivities at low and high energies (see Figure 15a). In order to agree with the cutflows of various analyses that require $b$-tagging, a reduction in the overall normalisation by $15 \%$ has been applied. In addition, the significant decrease of the signal efficiency at large energies has been manually added in order to get better agreement with experimental results.

Furthermore, the light quark jet rejection has been measured for two different $\eta$ regions, which we adapt in our parametrisations. We also perform a reduction in the light-quark tagging rates (20\%) in order to better agree with experimental cutflows.

Since the $p_{T}$ dependent distributions are given for a particular working point $\bar{\epsilon}_{\mathrm{S}}=0.7$, we linearly rescale the functions to the given chosen signal efficiency $\bar{\epsilon}_{\mathrm{S}}$, or the corresponding background efficiency given by the ROC curves $\left(p_{T}\right.$ in $\left.\mathrm{GeV}\right)$ :

$$
\begin{aligned}
& \epsilon_{\mathrm{S}}\left(p_{\mathrm{T}}\right)=\frac{\bar{\epsilon}_{\mathrm{S}} \cdot 0.85}{0.7}\left(0.552+\frac{0.210}{1+\mathrm{e}^{-0.123 \cdot\left(p_{\mathrm{T}}-47.6\right)}}\right) \frac{0.7+0.05 \cdot \mathrm{e}^{-\frac{p_{\mathrm{T}}}{308}}}{0.75} \begin{cases}1 & \text { if } p_{\mathrm{T}} \leq 100 \\
1-7 \times 10^{-4} \cdot\left(p_{\mathrm{T}}-100\right) & \text { if } p_{\mathrm{T}}>100\end{cases} \\
& \epsilon_{\text {light }}\left(p_{\mathrm{T}}, \eta\right)=\frac{\bar{r}_{\text {light }}(0.7) \times 0.8}{\bar{r}_{\text {light }}\left(\bar{\epsilon}_{\mathrm{S}}\right)}\left\{\begin{array}{l}
1.06 \times 10^{-2}+6.47 \times 10^{-6} \cdot p_{\mathrm{T}}^{2}+4.03 \times 10^{-8} \cdot p_{\mathrm{T}}^{4} \quad \text { if }|\eta|<1.3, \\
\left.6.61 \times 10^{-3}+6.49 \times 10^{-5} \cdot p_{\mathrm{T}}^{2}-3.12 \times 10^{-8} \cdot p_{\mathrm{T}}^{4} \quad \text { if } 1.3 \leq|\eta|<2.5\right),
\end{array}\right. \\
& \epsilon_{\mathrm{c}}\left(p_{\mathrm{T}}\right)=\frac{\bar{r}_{\mathrm{c}}(0.7) \times 0.4}{\bar{r}_{\mathrm{c}}\left(\bar{\epsilon}_{\mathrm{S}}\right)} \frac{0.461}{1+\mathrm{e}^{-0.0464 \cdot\left(p_{\mathrm{T}}-20.4\right)}} \text {. }
\end{aligned}
$$

\footnotetext{
24 The tagging probability for jets containing $D^{*}$ mesons is roughly 2 times better than for 'normal' c-quarks. Using the cutflows from various analyses we have tuned this parameter to 0.4 to be in agreement with the ATLAS results.
} 


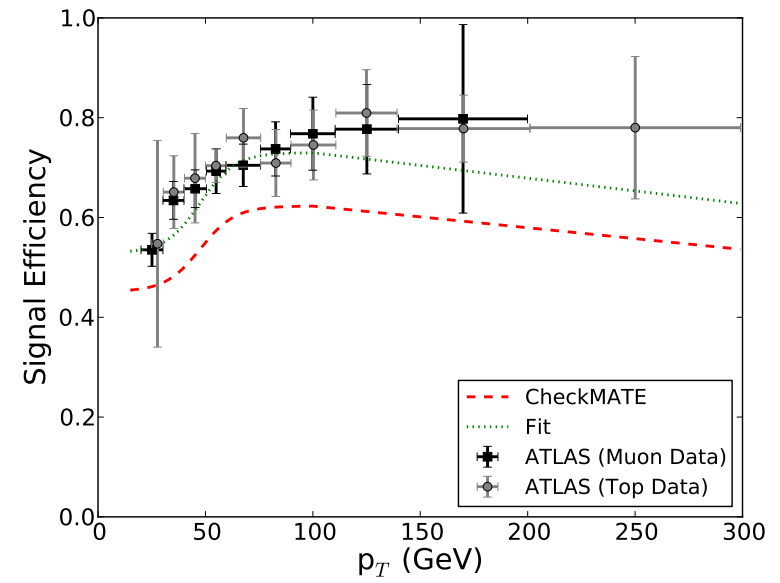

(a) Signal efficiencies for b-tagging, determined by combining the information of two different search channels for $\bar{\epsilon}_{\mathrm{S}}=0.7$ [52, 63].

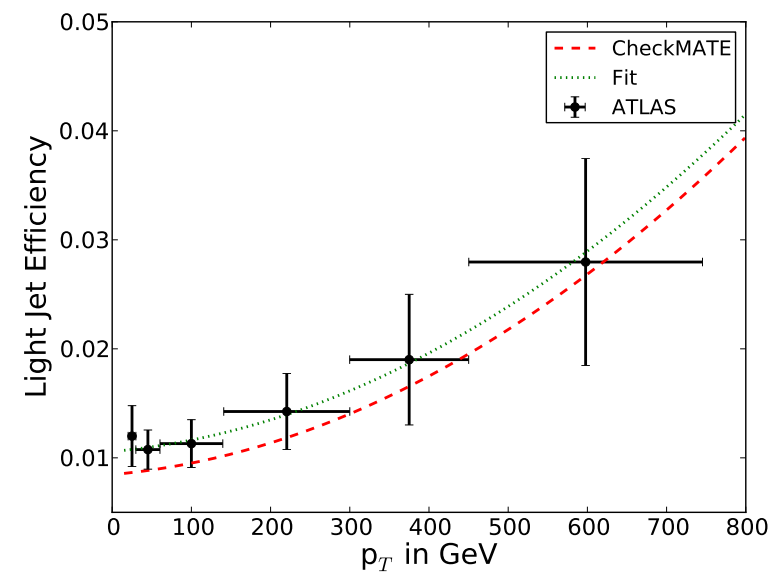

(c) B-tagging efficiency of jets containing light quarks for $\bar{\epsilon}_{\mathrm{S}}=0.7$ and $|\eta|<1.3$ [51].

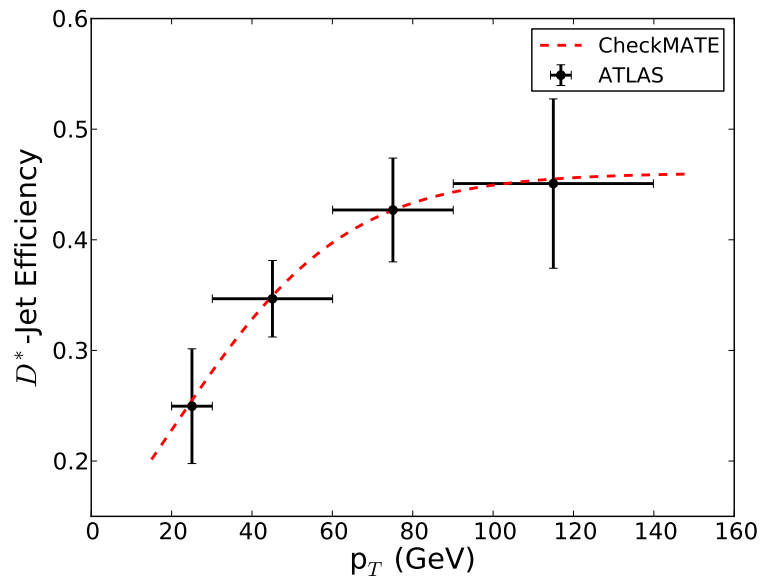

(b) B-tagging efficiency of jets containing $D^{*}$ mesons for $\bar{\epsilon}_{\mathrm{S}}=0.752$. The inclusive efficiency on $\mathrm{c}-$ jets is assumed to be $40 \%$ of this $D^{*}$ efficiency.

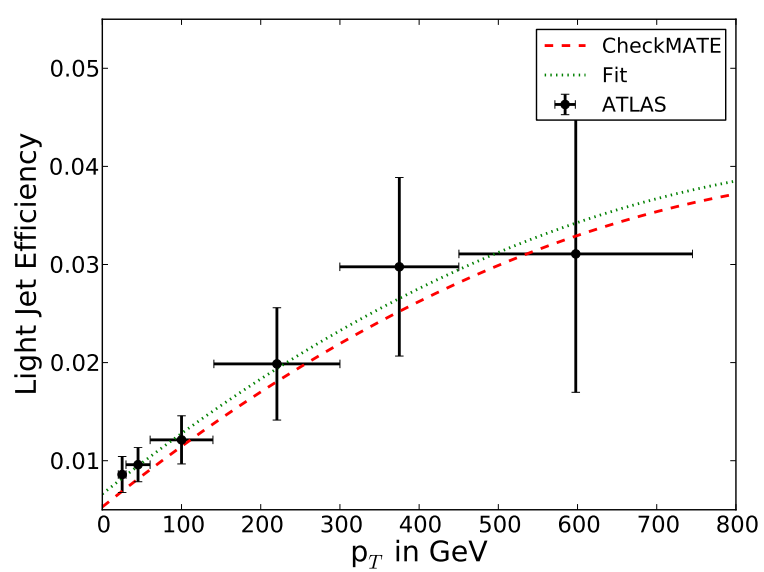

(d) Same as (c) for $1.3 \leq|\eta|<2.5$

FIG. 15: Dependence on the signal and light-jet / $c$-jet background efficiencies for b-tagging on the transverse momentum of the jet candidate.

\section{Tau-Tagger}

Analogously to the tagging of jets containing $b$-quarks, there exist algorithms to distinguish jets that originated from a hadronically decaying $\tau$ lepton from those that originated from quarks or gluons. Due to charge conservation, the $\tau$ lepton can only decay into an odd number of charged objects - mostly into 1 or 3 - called prongs. Since the structures of the resulting jets look rather different, identification algorithms usually differentiate between these two cases and hence there are individual efficiencies for each.

We show the efficiencies for signal and (light parton-)background with respect to the momentum of the jet candidate in Figure 16. Again, three common efficiency working points — 'loose, 'medium' and 'tight' — are used in most analyses, and we show results for each of the three. The functional descriptions of the data points are shown in Equations (C10) and C11, with the corresponding numerical values listed in Tables IIIa and IIIb. For background 
and 3-prong jets, these are the final efficiency functions Checkmate uses. Note that these efficiencies do not depend on $\eta$, but $|\eta|<2.5$ is always implied to fall within the angular coverage of the tracking detectors.

In addition to QCD jets, there is a second type of background which has to be taken into account: Electrons, which have been reconstructed as jets, can resemble 1-prong tau decays and need a separate tagging algorithm, which has been specifically tuned to reject these electron jet\$2 ${ }^{25}$. Efficiencies with respect to this algorithm depend on both transverse momentum and pseudorapidity as shown in Figures 17a and 17b. We follow the same approach as for the electron efficiencies, i.e. we take the absolute value from the momentum dependent efficiency distribution and multiply

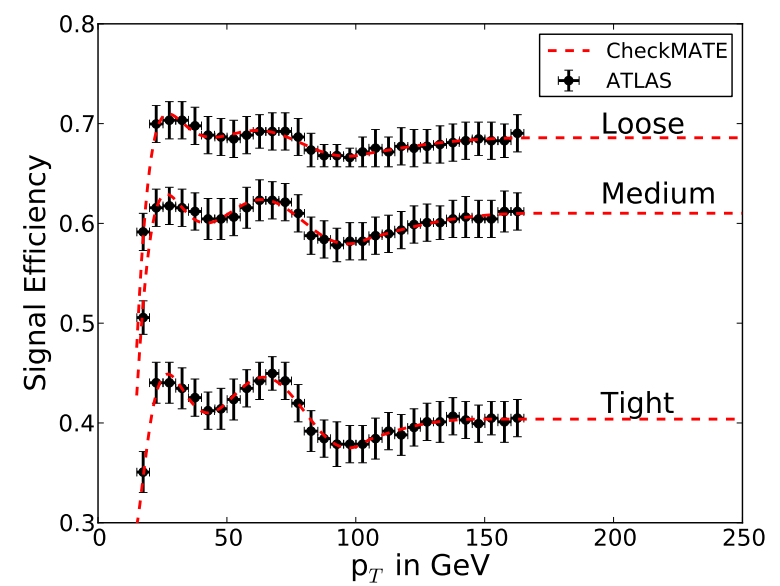

(a) Signal efficiencies for 1-prong candidates.

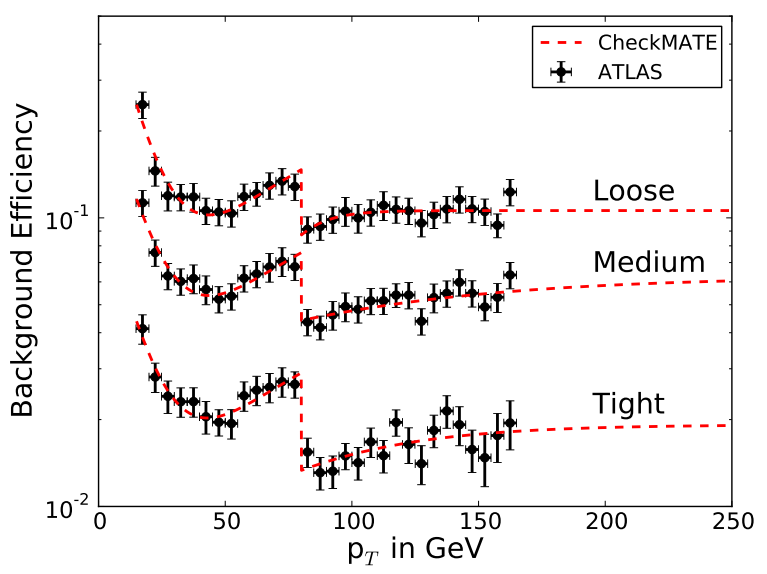

(c) Background efficiencies for 1-prong candidates.

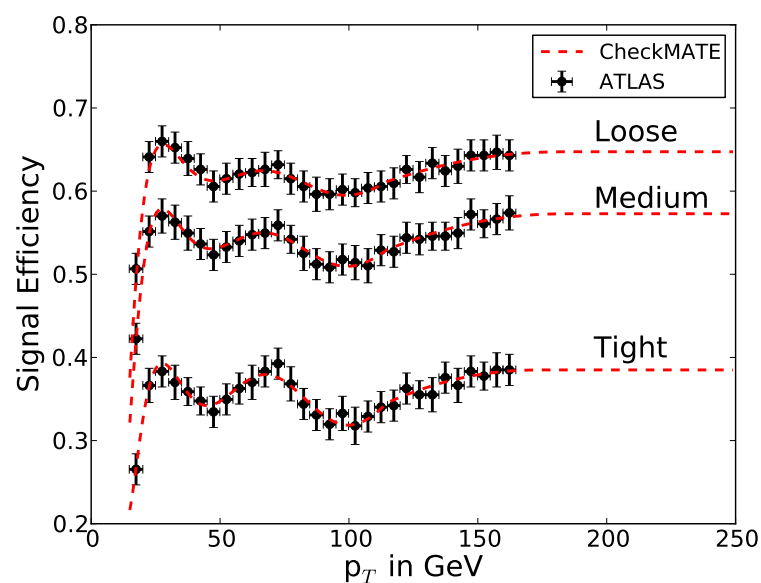

(b) Signal efficiencies for 3-prong candidates.

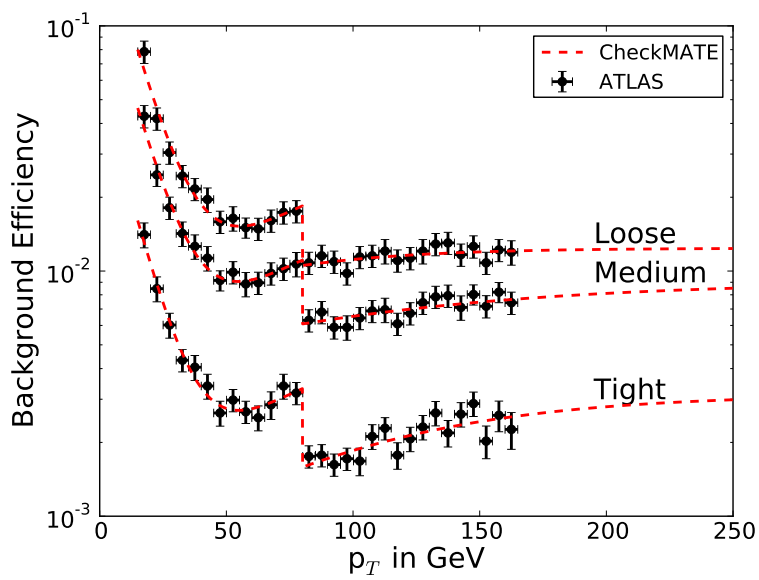

(d) Background efficiencies for 3-prong candidates.

FIG. 16: Signal and background efficiencies used for the tau-tagging discrimination against QCD jets. The results differentiate between jet candidates with one and with three reconstructed tracks (prongs) and the three most commonly used working points 'loose', 'medium' and 'tight 64. Explicit functions for the CheckMATE parametrisations are shown in Equations (C10) and (C11).

\footnotetext{
25 We only consider the impact on the signal efficiencies of this algorithm. In particular, we did not implement a specific mistagging efficiency for electrons. With the given Delphes code structure, any electron that fails the identification efficiency cut will be counted as a jet. Then, it will be tagged according to the corresponding background efficiency in Equation C11.
} 


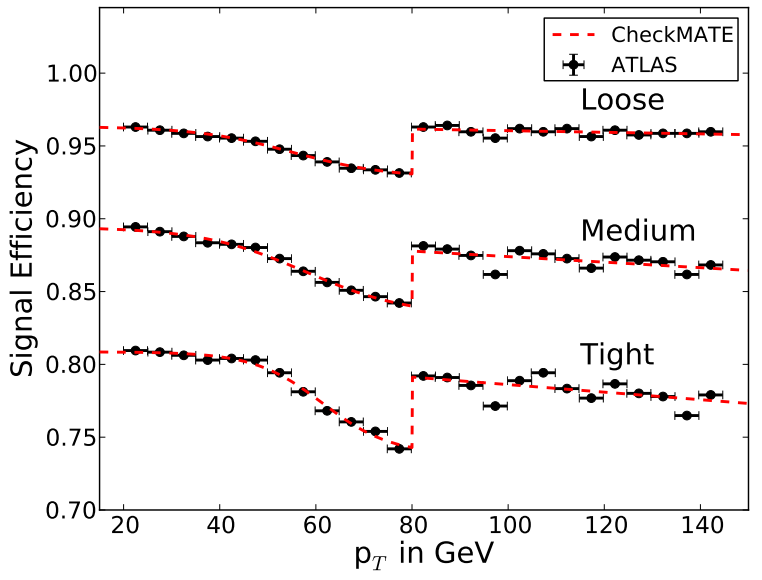

(a) Signal efficiency with respect to the candidate's transverse momentum.

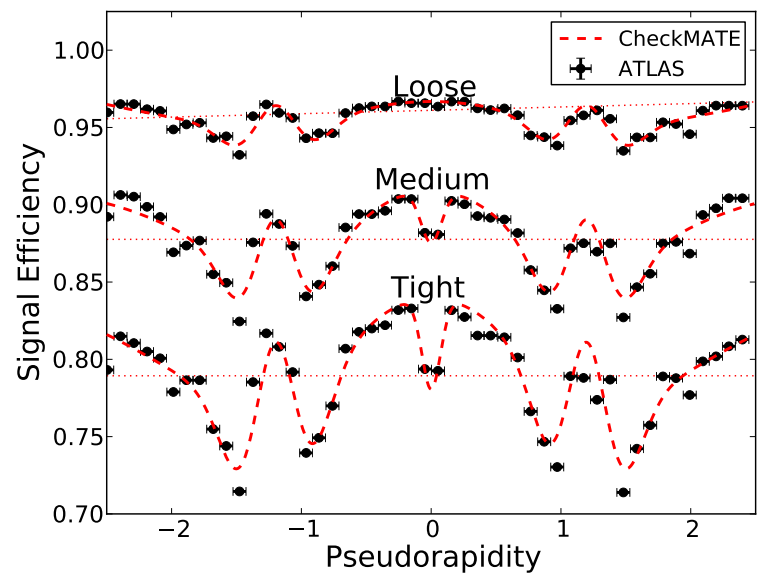

(b) Signal efficiency with respect to the candidate's pseudorapidity.

FIG. 17: Signal efficiencies used for the tau-tagging discrimination against electrons 64. These only affect 1-prong candidates, but differ between the different considered working points. Explicit functions for the CheckMATE parametrisations are shown in Equations (C12) and (C13). Dashed lines denote the respective average efficiency value.

with the pseudorapidity distribution normalised to an average efficiency value of 1 . The corresponding functions are shown in Equations $(\mathrm{C} 12)$ and $(\mathrm{C} 13)$ and Tables IIIc and IIId. We show a final combination of these with the 1-prong signal efficiency from before in Figures $18 \mathrm{a}$ to $18 \mathrm{c}$ for each of the three working points $\left(p_{T}\right.$ in $\left.\mathrm{GeV}\right)$ :

$$
\begin{aligned}
& \epsilon_{\mathrm{S}}\left(p_{\mathrm{T}}\right)=\epsilon_{0}+A_{1} p_{\mathrm{T}}^{\alpha} e^{-\lambda_{1} p_{\mathrm{T}}^{\beta}}+A_{2}\left\{\begin{array}{ll}
\left(p_{\mathrm{T}}-80\right)^{2} e^{-\lambda_{2}\left(p_{\mathrm{T}}-80\right)} & \text { if } p_{\mathrm{T}}<80+\frac{2}{\lambda_{2}}, \\
\frac{4}{\lambda_{2}^{2}} e^{-2} & \text { if } p_{\mathrm{T}} \geq 80+\frac{2}{\lambda_{2}},
\end{array}\right\}+ \\
& A_{3}\left\{\begin{array}{ll}
\frac{\sin \left(\omega\left(p_{\mathrm{T}}-15\right)+\phi\right)}{p_{\mathrm{T}}^{\gamma}} & \text { if } p_{\mathrm{T}}<105, \\
\frac{\sin (90 \omega+\phi)}{105^{\gamma}} & \text { if } p_{\mathrm{T}} \geq 105,
\end{array}\right\} \\
& \epsilon_{\mathrm{B}}\left(p_{\mathrm{T}}\right)=\left\{\begin{array}{ll}
A_{1} \mathrm{e}^{-\lambda_{1} p_{\mathrm{T}}}+m \cdot p_{\mathrm{T}} & \text { if } p_{\mathrm{T}}<80, \\
A_{2}\left(1-\frac{1}{1+e^{\lambda_{2}\left(p_{\mathrm{T}}-p_{\mathrm{T}}^{0}\right)}}\right) & \text { if } p_{\mathrm{T}} \geq 80,
\end{array}\right\} \\
& \epsilon_{\mathrm{S}}^{\text {el. veto }}\left(p_{\mathrm{T}}\right)= \begin{cases}\epsilon_{0}+\frac{k}{1+e^{\lambda \cdot\left(p_{\mathrm{T}}-p_{\mathrm{T}}^{0}\right)}} & \text { if } p_{\mathrm{T}} \leq 80 \\
-m \cdot p_{\mathrm{T}}+n & \text { if } p_{\mathrm{T}}>80\end{cases} \\
& \epsilon_{\mathrm{S}}^{1 \mathrm{p} \text { el. rej. (norm) }}(\eta)=\frac{1}{\langle\epsilon\rangle}\left(\epsilon_{0}-A_{1} \cdot \mathrm{e}^{-\frac{(|\eta|-1.2)^{2}}{\sigma_{1}^{2}}}+A_{2} \cdot \mathrm{e}^{-\frac{(|\eta|-1.2)^{2}}{\sigma_{2}^{2}}}-A_{3} \cdot \frac{\sin (\eta)}{\eta} \mathrm{e}^{-\frac{\eta^{2}}{\sigma_{3}^{2}}}+A_{4} \cdot \eta^{2} \mathrm{e}^{-\frac{\eta^{2}}{\sigma_{4}^{2}}}\right) \text {. }
\end{aligned}
$$




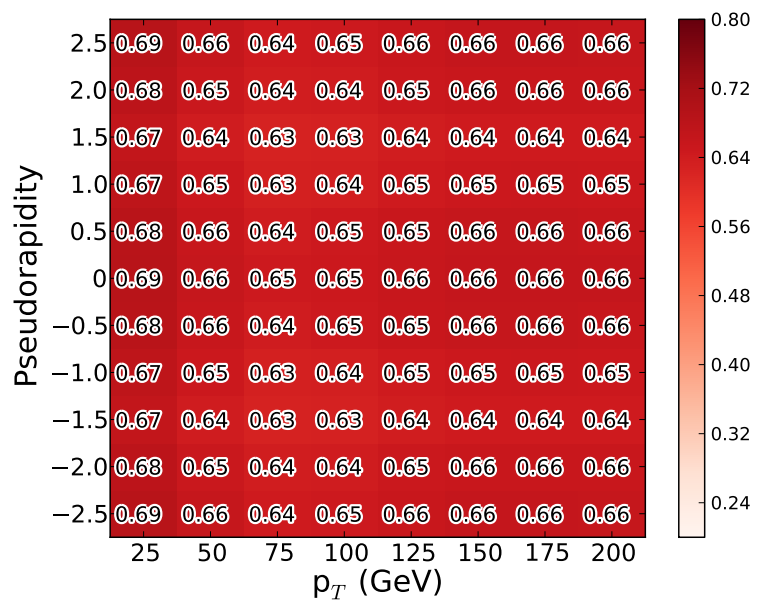

(a) Loose

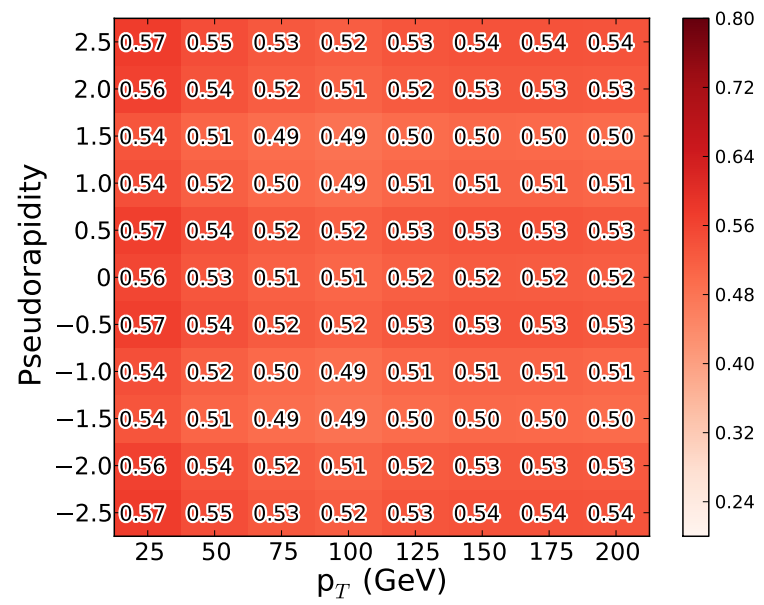

(b) Medium

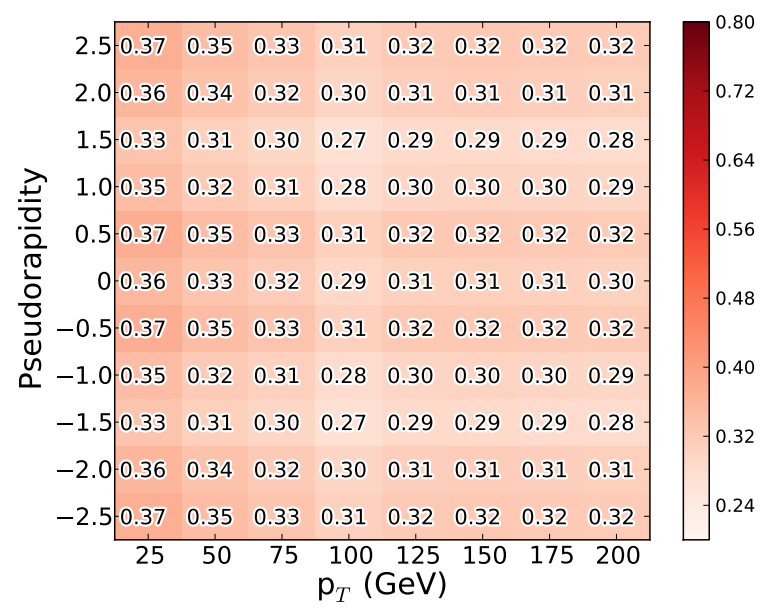

\section{(c) Tight}

FIG. 18: Total signal 1-prong efficiencies for 1-prong signal candidates, combining the contributions from rejections against QCD jets and electrons. 


\begin{tabular}{lccccccccccc}
\hline \hline & $\epsilon_{0}$ & $A_{1}$ & $\alpha$ & $\lambda_{1}$ & $\beta$ & $A_{2}$ & $\lambda_{2}$ & $A_{3}$ & $\omega$ & $\phi$ \\
\hline 1 prong, loose & 0.0223 & -2.55 & 1.16 & 0.427 & 0.846 & $2.14 \times 10^{-5}$ & 0.670 & 100 & 0.0974 & 2.34 & 2.04 \\
1 prong, medium & 0.0223 & -2.69 & 1.23 & 0.483 & 0.791 & $3.46 \times 10^{-5}$ & 0.586 & 100 & 0.0997 & 2.23 & 1.91 \\
1 prong, tight & 0.0292 & -2.78 & 1.17 & 0.377 & 0.799 & $6.17 \times 10^{-5}$ & 0.388 & 100 & 0.101 & 2.16 & 1.79 \\
3 prong, loose & 0.0192 & -6.44 & 0.143 & 0.106 & 1.08 & $4.22 \times 10^{-5}$ & 0.594 & 99.6 & 0.0958 & 2.09 & 1.92 \\
3 prong, medium & 0.0191 & -2.32 & 0.924 & 0.233 & 0.940 & $5.00 \times 10^{-5}$ & 0.510 & 100 & 0.0950 & 2.18 & 1.86 \\
3 prong, tight & 0.0212 & -2.29 & 0.671 & 0.104 & 1.096 & $6.30 \times 10^{-5}$ & 0.324 & 100 & 0.0971 & 2.09 & 1.78 \\
\hline \hline
\end{tabular}

(a) Parameters corresponding to Equation C10 for different signal working points and both 1-prong and 3-prong candidates.

\begin{tabular}{|c|c|c|c|c|c|c|}
\hline & $A_{1}$ & $\lambda_{1}$ & $m$ & $A_{2}$ & $\lambda_{2}$ & $p_{\mathrm{T}}^{0}$ \\
\hline 1 prong, loose & 0.717 & $7.89 \times 10^{-2}$ & $1.82 \times 10^{-3}$ & 0.106 & $9.73 \times 10^{-2}$ & $\frac{P 1}{64.3}$ \\
\hline 1 prong, medium & 0.301 & $7.20 \times 10^{-2}$ & $9.35 \times 10^{-4}$ & $6.27 \times 10^{-2}$ & $1.44 \times 10^{-2}$ & 20.0 \\
\hline 1 prong, tight & 0.117 & $7.42 \times 10^{-2}$ & $3.59 \times 10^{-4}$ & $1.92 \times 10^{-2}$ & $2.47 \times 10^{-2}$ & 46.8 \\
\hline 3 prong, loose & 0.265 & $8.27 \times 10^{-2}$ & $2.26 \times 10^{-4}$ & $1.24 \times 10^{-2}$ & $2.28 \times 10^{-2}$ & 4.08 \\
\hline 3 prong, medium & 0.154 & $8.32 \times 10^{-2}$ & $1.36 \times 10^{-4}$ & $9.06 \times 10^{-3}$ & $1.19 \times 10^{-2}$ & 20.0 \\
\hline 3 prong, tight & $5.79 \times 10^{-2}$ & $8.80 \times 10^{-2}$ & $4.08 \times 10^{-5}$ & $3.14 \times 10^{-3}$ & $1.73 \times 10^{-2}$ & 78.9 \\
\hline
\end{tabular}

(b) Parameters corresponding to Equation C11 for different working points and both 1-prong and 3-prong candidates.

\begin{tabular}{|c|c|c|c|c|c|c|c|c|c|}
\hline & $\epsilon_{0}$ & $A_{1}$ & $\sigma_{1}$ & $A_{2}$ & $\sigma_{2}$ & $A_{3}$ & $\sigma_{3}$ & $A_{4}$ & $\sigma_{4}$ \\
\hline loose & 0.94 & 0.875 & 0.294 & 0.886 & 0.286 & $-2.68 \times 10^{-2}$ & 1.16 & $4.19 \times 10^{-3}$ & 10.9 \\
\hline medium & 0.91 & 0.283 & 0.303 & 0.309 & 0.256 & $3.46 \times 10^{-2}$ & 0.113 & $-7.96 \times 10^{-2}$ & 1.25 \\
\hline tight & 0.84 & 0.472 & 0.304 & 0.503 & 0.258 & $5.94 \times 10^{-2}$ & 0.102 & $-8.51 \times 10^{-2}$ & 1.42 \\
\hline
\end{tabular}

(c) Parameters corresponding to Equation C12 for different working points (1-prong only).

\begin{tabular}{lcccccc}
\hline \hline & $\langle\epsilon\rangle$ & $\epsilon_{0}$ & $\mathrm{k}$ & $\lambda$ & $p_{\mathrm{T}}^{0}$ & $m$ \\
\hline loose & 0.956 & 0.928 & $3.54 \times 10^{-2}$ & 0.0994 & 55.1 & $5.52 \times 10^{-5}$ \\
medium & 0.878 & 0.833 & $6.13 \times 10^{-2}$ & 0.0922 & 57.8 & $1.90 \times 10^{-4}$ \\
tight & 0.789 & 0.738 & $7.06 \times 10^{-2}$ & 0.140 & 61.4 & $2.59 \times 10^{-4}$ \\
\hline \hline
\end{tabular}

(d) Parameters corresponding to Equation (C13) for different working points (1-prong only).

TABLE III: Numerical parameter values for the various efficiency distributions defined in Equations C10 to C13. 


\section{Appendix D: Analysis Validation}

\section{1. $\quad$ atlas_conf_2012_104}

1 lepton and $\mathbb{E}_{T}$. 27]

Energy: $8 \mathrm{TeV}$

Luminosity: $5.8 \mathrm{fb}^{-1}$

Validation notes:

- Validation has been performed versus the published CMSSM (mSUGRA) parameter scan.

- The exclusion in Checkmate is slightly weaker than the published ATLAS result, since ATLAS uses a different limit setting procedure. Checkmate only uses the signal region with the best expected sensitivity to set the limit. However, in this search ATLAS used a combined likelihood including all signal and control regions.

MSUGRA/CMSSM Exclusion

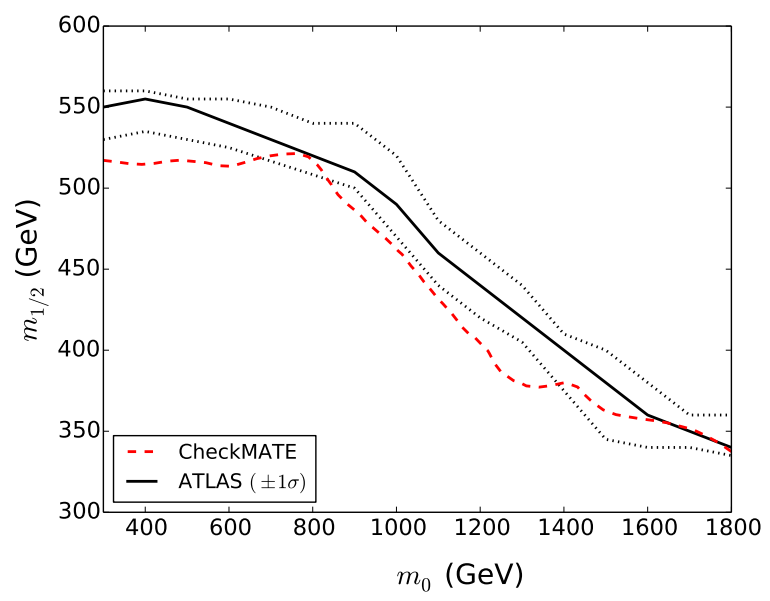

FIG. 19: Exclusion curve for general supersymmetric particle production in the CMSSM (mSUGRA) for atlas_conf_2012_104. 


\section{2. atlas_conf_2012_147}

Monojet search, 28]

Energy: $8 \mathrm{TeV}$

Luminosity: $10.5 \mathrm{fb}^{-1}$

Validation notes:

- Validation was performed against standard model $W$ and $Z$ samples in signal region distributions of leading jet $p_{\mathrm{T}}$ and $\mathbb{E}_{T}$.

- The validation finishes with the hardest jet $p_{\mathrm{T}}<600 \mathrm{GeVdue}$ to finite Monte-Carlo statistics.

- No validation has yet been performed with parameter scans.
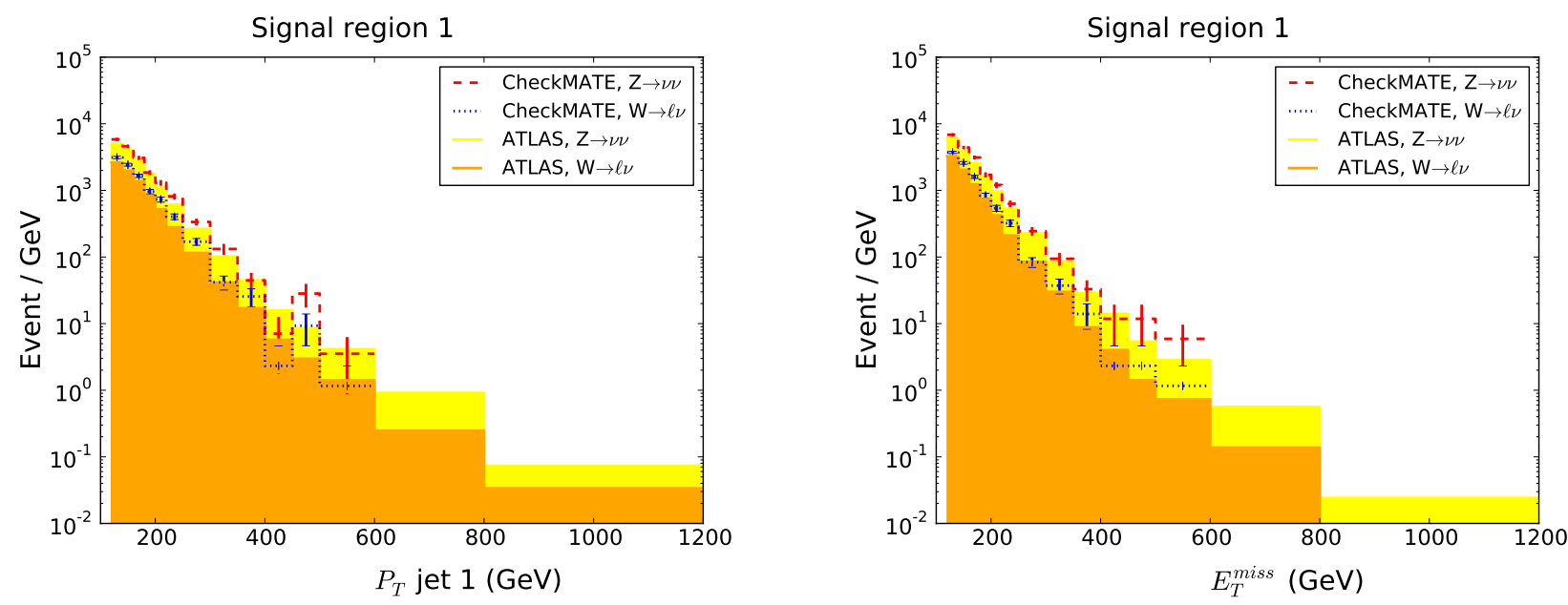

FIG. 20: Distributions for leading jet $p_{\mathrm{T}}$ (left) and $\mathbb{E}_{T}$ (right) for standard model production of $W$ and $Z$ plus jets in signal region 1 of atlas_conf_2012_147. The ATLAS $W / Z$ plus jets backgrounds are estimated using Monte-Carlo event samples normalised using data in control regions.
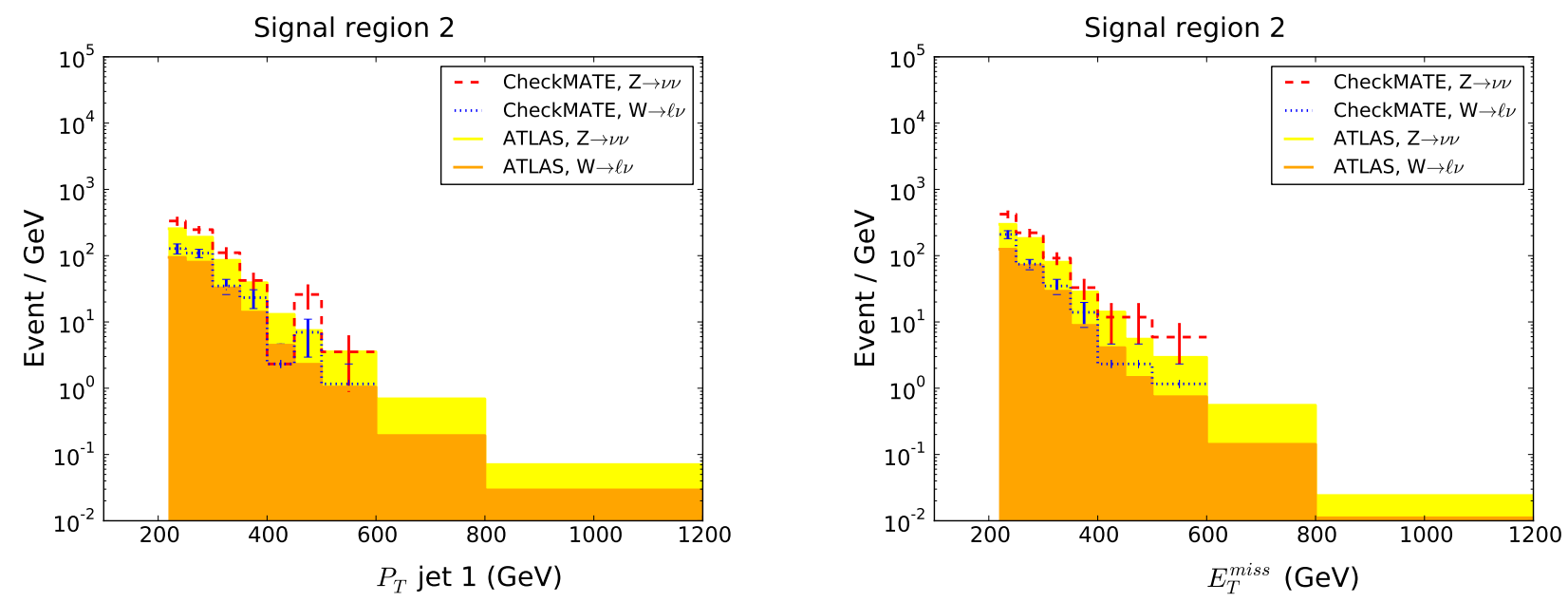

FIG. 21: Distributions for leading jet $p_{\mathrm{T}}$ (left) and $\mathbb{E}_{T}$ (right) for standard model production of $W$ and $Z$ plus jets in signal region 2 of atlas_conf_2012_147. The ATLAS $W / Z$ plus jets backgrounds are estimated using Monte-Carlo event samples normalised using data in control regions. 


\section{3. atlas_conf_2013_024}

All-hadronic stop search, $t \bar{t}+E_{T}^{\text {miss }},[29$

Energy: $8 \mathrm{TeV}$

Luminosity: $20.5 \mathrm{fb}^{-1}$

Validation notes:

- Validation has been performed versus all published cutflows and a simplified model consisting of pure stop production followed by the decay $\tilde{t} \rightarrow t \tilde{\chi}_{1}^{0}$.

\begin{tabular}{|c|c|c|c|c|}
\hline $\begin{array}{l}\text { Process } \\
\text { Point }\end{array}$ & & & & \\
\hline Top Polarization & & & & \\
\hline Source & ATLAS & CheckMATE & ATLAS & Checkmate \\
\hline No selection & 507.3 & 507.3 & 507.3 & 507.3 \\
\hline Trigger & 468.0 & 469.7 & 467.8 & 468.8 \\
\hline Primary vertex * & 467.8 & - & 467.4 & - \\
\hline Event Cleaning * & 459.0 & - & 459.6 & - \\
\hline Muon veto & 381.2 & 380.1 & 382.5 & 380.4 \\
\hline Electron veto & 284.4 & 297.6 & 292.3 & 302.2 \\
\hline $\mathbb{E}_{T}>130 \mathrm{GeV}$ & 263.1 & 275.4 & 270.1 & 277.7 \\
\hline Jet multiplicity and $p_{\mathrm{T}}$ & 97.7 & 95.0 & 92.2 & 87.3 \\
\hline $\mathbb{E}_{T}^{\text {track }}>30 \mathrm{GeV}$ & 96.3 & 93.5 & 90.5 & 85.9 \\
\hline$\Delta \phi\left(\mathbb{E}_{T}, \mathbb{E}_{T}^{\text {track }}\right)<\pi / 3$ & 90.3 & 89.4 & 84.3 & 82.2 \\
\hline$\Delta \phi\left(\right.$ jet, $\left.\mathbb{E}_{T}^{\text {track }}\right)<\pi / 3$ & 77.1 & 76.0 & 72.0 & 69.2 \\
\hline Tau veto & 67.4 & 66.6 & 61.9 & 59.3 \\
\hline $2 \geq b$-tagged jets & 29.5 & 28.6 & 31.5 & 27.9 \\
\hline$m_{T}\left(b-j e t, \mathbb{E}_{T}\right)>175 \mathrm{GeV}$ & 20.2 & 20.2 & 23.6 & 21.3 \\
\hline $80 \mathrm{GeV}<m_{j j j}^{0}<175 \mathrm{GeV}$ & 17.8 & 18.4 & 20.4 & 19.3 \\
\hline $80 \mathrm{GeV}<m_{j j j}^{1}<175 \mathrm{GeV}$ & 10.9 & 10.8 & 11.9 & 10.9 \\
\hline $\mathbb{E}_{T}>150 \mathrm{GeV}$ & 10.8 & 10.7 & 11.8 & 11.8 \\
\hline $\mathbb{E}_{T}>200 \mathrm{GeV}(\mathrm{SR} 1)$ & $10.3 \pm 0.1$ & $10.2 \pm 0.2$ & $11.2 \pm 0.1$ & $10.5 \pm 0.2$ \\
\hline $\mathbb{E}_{T}>250 \mathrm{GeV}$ & 9.2 & 9.3 & 10.0 & 9.3 \\
\hline $\mathbb{E}_{T}>300 \mathrm{GeV}(\mathrm{SR} 2)$ & $7.8 \pm 0.1$ & $8.1 \pm 0.1$ & $8.3 \pm 0.1$ & $8.1 \pm 0.1$ \\
\hline $\mathbb{E}_{T}>350 \mathrm{GeV}(\mathrm{SR} 3)$ & $6.1 \pm 0.1$ & $6.2 \pm 0.1$ & $6.6 \pm 0.1$ & $6.5 \pm 0.1$ \\
\hline
\end{tabular}

TABLE IV: In the left (right) column, the tops from the stop decay are right-handed (left-handed) in 95\% (100\%) of the decays. Shown are the number of events after each selection cut, normalised to $20.5 \mathrm{fb}^{-1}$. Final error is from Monte Carlo statistics for both ATLAS and CheckMATE. * No vertex finding or event cleaning is performed by CheckMATE. Instead, a flat efficiency factor is included to account for these effects. 


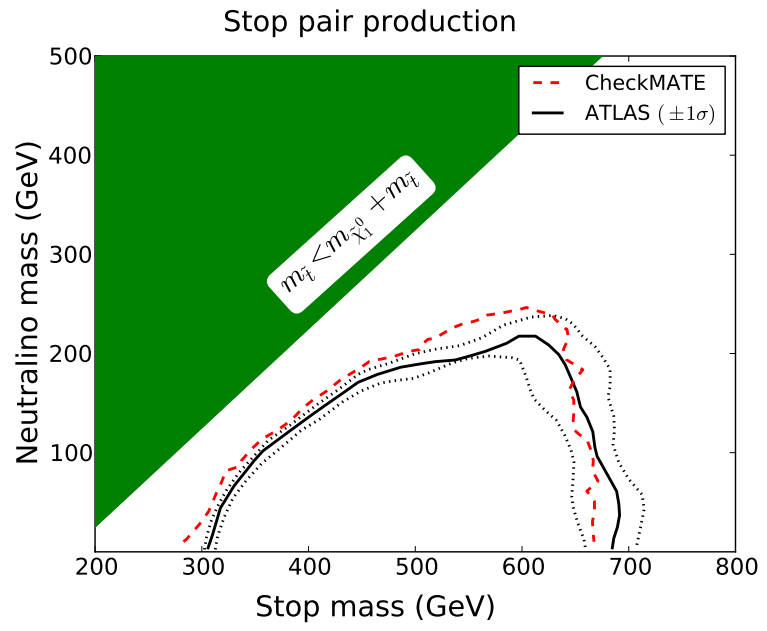

FIG. 22: Exclusion curve for the stop pair production with simplified decay, $\tilde{t} \rightarrow t \tilde{\chi}_{1}^{0}$ for analysis atlas_conf_2013_024. The top produced in the decay has right-handed polarization in $95 \%$ of the decays. 


\section{4. atlas_conf_2013_035}

Trilepton, 0 jets $+E_{T}^{m i s s}$, 30.

Energy: $8 \mathrm{TeV}$

Luminosity: $20.7 \mathrm{fb}^{-1}$

Validation notes:

- Validation has been performed versus all published cutflows.

\begin{tabular}{|c|c|c|c|c|c|c|}
\hline \multirow{2}{*}{$\begin{array}{l}\text { Process } \\
\text { Point }\end{array}$} & \multicolumn{6}{|c|}{$\tilde{\chi}_{2}^{0}+\tilde{\chi}_{1}^{ \pm}$production; decay via $W / Z$ or slepton } \\
\hline & \multicolumn{2}{|c|}{$\begin{array}{c}\text { Simplified } \tilde{\ell}_{L} \\
m_{\tilde{\chi}_{1}^{0}}=157.5 \mathrm{GeV} \\
m_{\tilde{\chi}_{2}^{0}}=m_{\tilde{\chi}_{1}^{ \pm}}=192.5 \mathrm{GeV} \\
m_{\tilde{\nu}}=m_{\tilde{l}_{L}}=\left(m_{\tilde{\chi}_{1}^{0}}+m_{\tilde{\chi}_{1}^{ \pm}}\right) / 2 \\
\text { SRnoZa }\end{array}$} & \multicolumn{2}{|c|}{$\begin{array}{c}\text { Simplified WZ } \\
m_{\tilde{\chi}_{1}^{0}}=75 \mathrm{GeV} \\
m_{\tilde{\chi}_{2}^{0}}=m_{\tilde{\chi}_{1}^{ \pm}}=150 \mathrm{GeV} \\
\text { SRnoZb }\end{array}$} & \multicolumn{2}{|c|}{$\begin{array}{c}\text { Simplified } \tilde{\ell}_{L} \\
m_{\tilde{\chi}_{1}^{0}}=0 \mathrm{GeV} \\
m_{\tilde{\chi}_{2}^{0}}=m_{\tilde{\chi}_{1}^{ \pm}}=500 \mathrm{GeV} \\
m_{\tilde{\nu}}=m_{\tilde{l}_{L}}=\left(m_{\tilde{\chi}_{1}^{0}}+m_{\tilde{\chi}_{1}^{ \pm}}\right) / 2 \\
\text { SRnoZc }\end{array}$} \\
\hline Source & ATLAS & CheckMATE & ATLAS & CheckMATE & ATLAS & CheckMATE \\
\hline Generated events & 25000 & 40000 & 20000 & 40000 & 40000 & 40000 \\
\hline Lepton multiplicity & 537.1 & 688.1 & 227.3 & 278.8 & 28.5 & 28.1 \\
\hline SFOS requirement & 536.3 & 615 & 226.5 & 272.5 & 28.1 & 27.9 \\
\hline b veto & 491.0 & 557.4 & 211.0 & 251 & 24.9 & 24.8 \\
\hline $\mathrm{Z}$ veto/request & 476.3 & 537 & 196.6 & 235 & 24.1 & 24 \\
\hline$E_{T}^{\mathrm{miss}}$ & 161.2 & 181.3 & 53.8 & 56.2 & 22.1 & 22 \\
\hline$m_{\mathrm{SFOS}}$ & 141.2 & 154.2 & 27.1 & 26.8 & - & - \\
\hline$m_{T}$ & - & - & - & - & 19.2 & 19 \\
\hline$p_{\mathrm{T}}^{\text {third lepton }}$ & - & - & - & - & $18.4 \pm 0.3$ & $18 \pm 0.3$ \\
\hline SRnoZc veto & $141.2 \pm 9.9$ & $154.2 \pm 8.1$ & $26.3 \pm 1.4$ & $26.8 \pm 1.0$ & - & - \\
\hline
\end{tabular}

TABLE V: Shown are the number of events after each selection cut, normalised to $20.7 \mathrm{fb}^{-1}$. Final error is from Monte Carlo statistics for both ATLAS and CheckMATE.

\begin{tabular}{|c|c|c|c|c|c|c|}
\hline \multirow{2}{*}{$\begin{array}{l}\text { Process } \\
\text { Point }\end{array}$} & \multicolumn{6}{|c|}{$\tilde{\chi}_{2}^{0}+\tilde{\chi}_{1}^{ \pm}$production; decay via $W / Z$ or slepton } \\
\hline & \multicolumn{2}{|c|}{$\begin{array}{c}\text { Simplified WZ } \\
m_{\tilde{\chi}_{1}^{0}}=0 \mathrm{GeV} \\
m_{\tilde{\chi}_{2}^{0}}=m_{\tilde{\chi}_{1}^{ \pm}}=100 \mathrm{GeV} \\
\text { SRZa }\end{array}$} & \multicolumn{2}{|c|}{$\begin{array}{c}\text { Simplified WZ } \\
m_{\tilde{\chi}_{1}^{0}}=0 \mathrm{GeV} \\
m_{\tilde{\chi}_{2}^{0}}=m_{\tilde{\chi}_{1}^{ \pm}}=150 \mathrm{GeV} \\
\text { SRZb }\end{array}$} & \multicolumn{2}{|c|}{$\begin{array}{c}\text { Simplified WZ } \\
m_{\tilde{\chi}_{1}^{0}}=0 \mathrm{GeV} \\
m_{\tilde{\chi}_{2}^{0}}=m_{\tilde{\chi}_{1}^{ \pm}}=250 \mathrm{GeV} \\
m_{\tilde{\nu}}=m_{\tilde{l}_{L}}=\left(m_{\tilde{\chi}_{1}^{0}}+m_{\tilde{\chi}_{1}^{ \pm}}\right) / 2 \\
\mathrm{SRZc}\end{array}$} \\
\hline Source & ATLAS & CheckMATE & ATLAS & CheckMATE & ATLAS & CheckMATE \\
\hline Generated events & 15000 & 40000 & 20000 & 40000 & 20000 & 40000 \\
\hline Lepton multiplicity & 1071.4 & 1118.9 & 259.8 & 276.9 & 40.0 & 44.2 \\
\hline SFOS requirement & 1067.5 & 1109.5 & 258.0 & 273.9 & 39.7 & 43.7 \\
\hline b veto & 989.4 & 1039 & 240.0 & 254.6 & 36.4 & 39.8 \\
\hline $\mathrm{Z}$ veto/request & 912.7 & 866.5 & 227.2 & 227.1 & 34.4 & 34.9 \\
\hline$E_{T}^{\mathrm{miss}}$ & 170.7 & 135.9 & 67.7 & 66.5 & 17.7 & 17.3 \\
\hline$m_{\mathrm{SFOS}}$ & - & - & - & - & - & - \\
\hline$m_{T}$ & $159.3 \pm 8.6$ & $133 \pm 5.2$ & $27.8 \pm 1.4$ & $26 \pm 1.0$ & $12.0 \pm 0.34$ & $11.2 \pm 0.23$ \\
\hline$p_{\mathrm{T}}^{\text {third lepton }}$ & - & - & - & - & - & - \\
\hline SRnoZc veto & - & - & - & - & - & - \\
\hline
\end{tabular}

TABLE VI: Shown are the number of events after each selection cut, normalised to $20.7 \mathrm{fb}^{-1}$. Final error is from Monte Carlo statistics for both ATLAS and CheckmATE. 


\section{5. atlas_conf_2013_047}

0 lepton $+\geq 2-6$ jets $+E_{T}^{\text {miss }}$, 31.

Energy: $8 \mathrm{TeV}$

Luminosity: $20.3 \mathrm{fb}^{-1}$

Validation notes:

- Validation has been performed versus all published cutflows.

- Additional validation has been performed with the parameter scan of the CMSSM (mSUGRA) model shown in Figure 9

\begin{tabular}{|c|c|c|c|c|c|c|}
\hline $\begin{array}{l}\text { Process } \\
\text { Point } \\
\text { Signal Region }\end{array}$ & $\begin{array}{r}m(\tilde{q}) \\
m\left(\tilde{\chi}_{1}^{0}\right) \\
\text { A-1 }\end{array}$ & $\begin{array}{l}50 \mathrm{GeV} \\
00 \mathrm{GeV} \\
\text { ium }\end{array}$ & $\begin{array}{r}\tilde{q} \tilde{q} \\
m(\tilde{q}) \\
m\left(\tilde{\chi}_{1}^{0}\right) \\
\text { A-1 }\end{array}$ & $\begin{array}{l}\text { ect } \\
50 \mathrm{GeV} \\
00 \mathrm{GeV} \\
\text { ium }\end{array}$ & $\begin{array}{r}m(\tilde{q}) \\
m\left(\tilde{\chi}_{1}^{0}\right) \\
\text { C- }\end{array}$ & $\begin{array}{l}62 \mathrm{GeV} \\
87 \mathrm{GeV} \\
\text { ium }\end{array}$ \\
\hline Source & ATLAS & CheckMATE & ATLAS & CheckMATE & ATLAS & CheckMATE \\
\hline Generated events & 20000 & 50000 & 5000 & 50000 & 5000 & 50000 \\
\hline Jet Cleaning * & 99.7 & - & 99.6 & - & 99.6 & - \\
\hline 0-lepton * & 89.9 & - & 98.5 & - & 98.2 & - \\
\hline $\mathbb{E}_{T}>160 \mathrm{GeV}^{*}$ & 15 & - & 89.9 & - & 80.7 & - \\
\hline$p_{\mathrm{T}}\left(j_{1}\right)>130 \mathrm{GeV}$ & 12.9 & 12.9 & 89.7 & 89.5 & 80.0 & 79.3 \\
\hline$p_{\mathrm{T}}\left(j_{2}\right)>130 \mathrm{GeV}$ & 9.0 & 8.4 & 87.4 & 87.1 & 75.6 & 75.3 \\
\hline$p_{\mathrm{T}}\left(j_{3}\right)>0-60 \mathrm{GeV}$ & 9.0 & 8.4 & 87.4 & 87.1 & 35.3 & 35.6 \\
\hline$p_{\mathrm{T}}\left(j_{4}\right)>0-60 \mathrm{GeV}$ & 9.0 & 8.4 & 87.4 & 87.1 & 11.5 & 11.3 \\
\hline$\Delta \phi\left(j_{i}>40, \mathbb{E}_{T}\right)>0.4$ & 7.0 & 6.8 & 79.2 & 79.0 & 10.1 & 9.9 \\
\hline$\Delta \phi\left(j_{i}>40 \mathrm{GeV}, \mathbb{E}_{T}\right)>0-0.2$ & 7.0 & 6.8 & 79.2 & 79.0 & 9.3 & 9.2 \\
\hline $\mathbb{E}_{T} / \sqrt{H_{T}}>0-15$ & 2.6 & 1.8 & 49.9 & 48.0 & 9.3 & 9.2 \\
\hline $\mathbb{E}_{T} / m_{\mathrm{eff}}\left(N_{j}\right)>0.15-0.4$ & 2.6 & 1.8 & 49.9 & 48.0 & 7.2 & 6.8 \\
\hline$m_{\mathrm{eff}}($ incl. $)>1-2.2 \mathrm{TeV}$ & $0.1 \pm 0.02$ & $0.08 \pm 0.01$ & $16.5 \pm 0.6$ & $18.3 \pm 0.2$ & $3.0 \pm 0.2$ & $3.1 \pm 0.1$ \\
\hline
\end{tabular}

TABLE VII: The cutflow is given as an absolute efficiency in $\%$ for each step of event selection. Final error is from Monte Carlo statistics for both ATLAS and CheckMATE. *Variable trigger efficiencies mean that the results are only comparable after both an $\mathbb{E}_{T}$ and jet $p_{T}$ cut have been applied.

\begin{tabular}{|c|c|c|c|c|c|c|}
\hline \multirow{3}{*}{$\begin{array}{l}\text { Process } \\
\text { Point } \\
\text { Signal Region } \\
\text { Source }\end{array}$} & \multicolumn{4}{|c|}{$\tilde{q} \tilde{g}$ direct } & \multirow{2}{*}{\multicolumn{2}{|c|}{$\begin{array}{c}\tilde{g} \tilde{g} \text { direct } \\
m(\tilde{g})=1162 \mathrm{GeV} \\
m\left(\tilde{\chi}_{1}^{0}\right)=337 \mathrm{GeV} \\
\mathrm{D}\end{array}$}} \\
\hline & \multicolumn{2}{|c|}{$\begin{array}{c}m(\tilde{g})=1425 \mathrm{GeV} \\
m\left(\tilde{\chi}_{1}^{0}\right)=525 \mathrm{GeV} \\
\text { B-medium }\end{array}$} & \multicolumn{2}{|c|}{$\begin{array}{c}m(\tilde{g})=1612 \mathrm{GeV} \\
m\left(\tilde{\chi}_{1}^{0}\right)=37 \mathrm{GeV} \\
\text { B-tight }\end{array}$} & & \\
\hline & ATLAS & CheckmATE & ATLAS & Checkmate & ATLAS & CheckMATE \\
\hline Generated events & 5000 & 50000 & 5000 & 50000 & 5000 & 50000 \\
\hline Jet Cleaning ${ }^{*}$ & 99.7 & - & 99.6 & - & 99.8 & - \\
\hline 0-lepton * & 98.0 & - & 98.8 & - & 98.5 & - \\
\hline $\mathbb{E}_{T}>160 \mathrm{GeV}^{*}$ & 93.3 & - & 95.9 & - & 88.9 & - \\
\hline$p_{\mathrm{T}}\left(j_{1}\right)>130 \mathrm{GeV}$ & 93.3 & 93.9 & 95.8 & 96.0 & 88.8 & 88.1 \\
\hline$p_{\mathrm{T}}\left(j_{2}\right)>130 \mathrm{GeV}$ & 92.4 & 92.7 & 95.2 & 95.1 & 88.8 & 88.1 \\
\hline$p_{\mathrm{T}}\left(j_{3}\right)>0-60 \mathrm{GeV}$ & 68.5 & 67.0 & 75.7 & 73.5 & 87.1 & 86.8 \\
\hline$p_{\mathrm{T}}\left(j_{4}\right)>0-60 \mathrm{GeV}$ & 68.5 & 67.0 & 75.7 & 73.5 & 74.1 & 74.4 \\
\hline$p_{\mathrm{T}}\left(j_{5}\right)>0-60 \mathrm{GeV}$ & 68.5 & 67.0 & 75.7 & 73.5 & 40.9 & 36.0 \\
\hline$\Delta \phi\left(j_{i}>40, \mathbb{E}_{T}\right)>0.4$ & 60.4 & 58.7 & 66.2 & 64.2 & 34.2 & 30.1 \\
\hline$\Delta \phi\left(j_{i}>40 \mathrm{GeV}, \mathbb{E}_{T}\right)>0-0.2$ & 60.4 & 58.7 & 66.2 & 64.2 & 28.6 & 25.9 \\
\hline $\mathbb{E}_{T} / m_{\mathrm{eff}}\left(N_{j}\right)>0.15-0.4$ & 44.8 & 41.8 & 31.8 & 28.1 & 22.1 & 18.9 \\
\hline$m_{\mathrm{eff}}($ incl. $)>1-2.2 \mathrm{TeV}$ & $27.5 \pm 0.7$ & $25.8 \pm 0.2$ & $22.8 \pm 0.7$ & $20.5 \pm 0.2$ & $13.4 \pm 0.5$ & $13.0 \pm 0.2$ \\
\hline
\end{tabular}

TABLE VIII: The cutflow is given as an absolute efficiency in \% for each step of event selection. Final error is from Monte Carlo statistics for both ATLAS and CheckMATE. * Variable trigger efficiencies mean that the results are only comparable after both an $\mathbb{E}_{T}$ and jet $p_{T}$ cut have been applied. 


\begin{tabular}{|c|c|c|c|c|}
\hline Process & \multicolumn{4}{|c|}{$\tilde{g} \tilde{g}$ one-step $\left(\tilde{g}\right.$ decay via $\left.\tilde{\chi}^{ \pm}\right)$} \\
\hline Signal Region & \multicolumn{2}{|c|}{$\begin{array}{c}m(\tilde{g})=1065 \mathrm{GeV} \\
m\left(\tilde{\chi}_{1}^{ \pm}\right)=785 \mathrm{GeV} \\
m\left(\tilde{\chi}_{1}^{0}\right)=525 \mathrm{GeV} \\
D\end{array}$} & \multicolumn{2}{|c|}{$\begin{array}{c}m(\tilde{g})=1265 \mathrm{GeV} \\
m\left(\tilde{\chi}_{1}^{ \pm}\right)=865 \mathrm{GeV} \\
m\left(\tilde{\chi}_{1}^{0}\right)=465 \mathrm{GeV} \\
\text { E-tight }\end{array}$} \\
\hline Source & ATLAS & CheckMATE & ATLAS & CheckMATE \\
\hline Generated events & 20000 & 50000 & 20000 & 50000 \\
\hline Jet Cleaning * & 99.8 & - & 99.8 & - \\
\hline 0-lepton * & 63.7 & - & 63.5 & - \\
\hline $\mathbb{E}_{T}>160 \mathrm{GeV}^{*}$ & 50.0 & - & 55.6 & - \\
\hline$p_{\mathrm{T}}\left(j_{1}\right)>130 \mathrm{GeV}$ & 49.3 & 47.7 & 55.6 & 54.4 \\
\hline$p_{\mathrm{T}}\left(j_{2}\right)>130 \mathrm{GeV}$ & 49.2 & 47.6 & 55.6 & 54.4 \\
\hline$p_{\mathrm{T}}\left(j_{3}\right)>0-60 \mathrm{GeV}$ & 48.6 & 47.1 & 55.4 & 54.2 \\
\hline$p_{\mathrm{T}}\left(j_{4}\right)>0-60 \mathrm{GeV}$ & 44.5 & 43.8 & 53.4 & 52.8 \\
\hline$p_{\mathrm{T}}\left(j_{5}\right)>0-60 \mathrm{GeV}$ & 34.4 & 34.8 & 46.3 & 46.6 \\
\hline$p_{\mathrm{T}}\left(j_{5}\right)>0-60 \mathrm{GeV}$ & 34.4 & 34.8 & 31.7 & 33.0 \\
\hline$\Delta \phi\left(j_{i}>40, \mathbb{E}_{T}\right)>0.4$ & 29.2 & 29.5 & 26.5 & 27.5 \\
\hline$\Delta \phi\left(j_{i}>40 \mathrm{GeV}, \mathbb{E}_{T}\right)>0-0.2$ & 24.6 & 24.7 & 21.3 & 22.4 \\
\hline $\mathbb{E}_{T} / m_{\mathrm{eff}}\left(N_{j}\right)>0.15-0.4$ & 21.6 & 21.2 & 12.0 & 11.2 \\
\hline$m_{\mathrm{eff}}($ incl. $)>1-2.2 \mathrm{TeV}$ & $2.0 \pm 0.1$ & $1.9 \pm 0.06$ & $7.9 \pm 0.2$ & $8.2 \pm 0.1$ \\
\hline
\end{tabular}

TABLE IX: The cutflow is given as an absolute efficiency in \% for each step of event selection. Final error is from Monte Carlo statistics for both ATLAS and Checkmate. *Variable trigger efficiencies mean that the results are only comparable after both an $\mathbb{E}_{T}$ and jet $p_{T}$ cut have been applied.
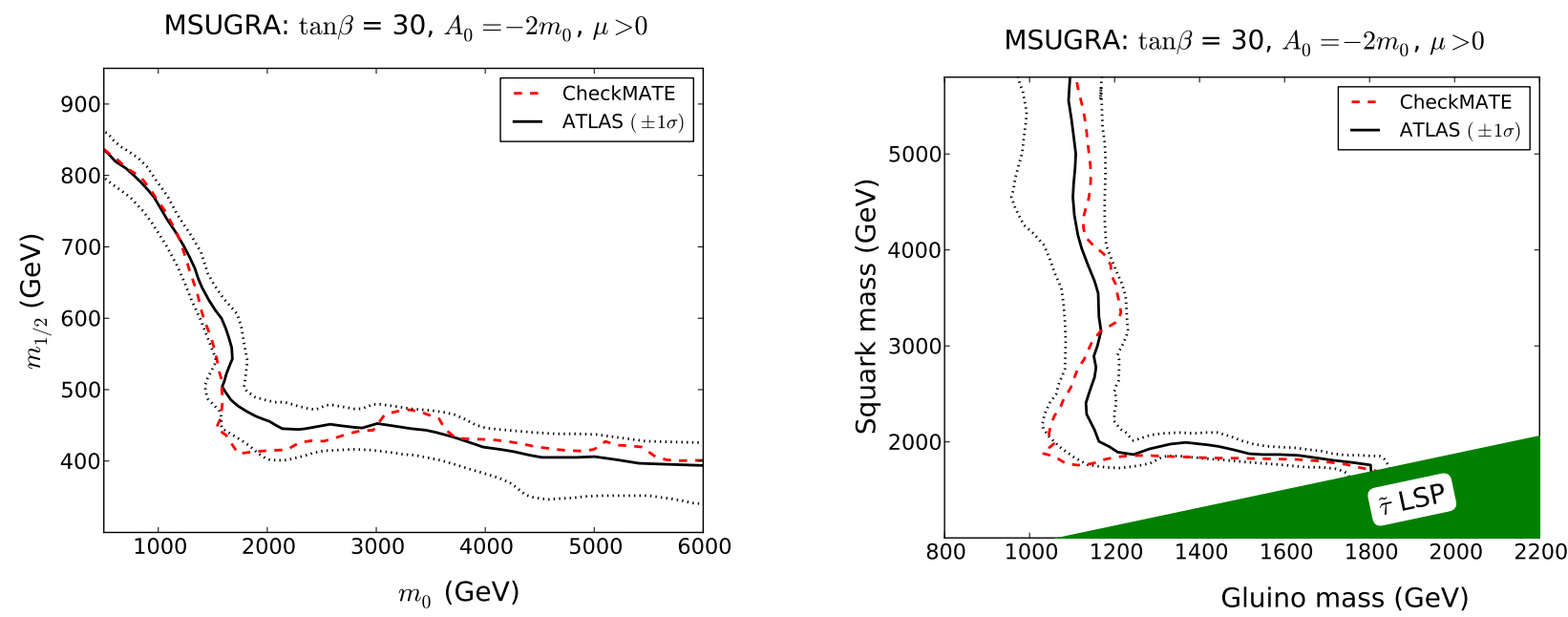

FIG. 23: Exclusion curve for the CMSSM (mSUGRA) model for analysis atlas_conf_2013_047. The exclusion in the parameter space $M_{0}, M_{1 / 2}$ (left) and $m_{\tilde{q}}, m_{\tilde{g}}$ are shown (right). 
Squark Gluino Neutralino Model, $M_{\chi_{1}^{0}}=0 \mathrm{GeV}$

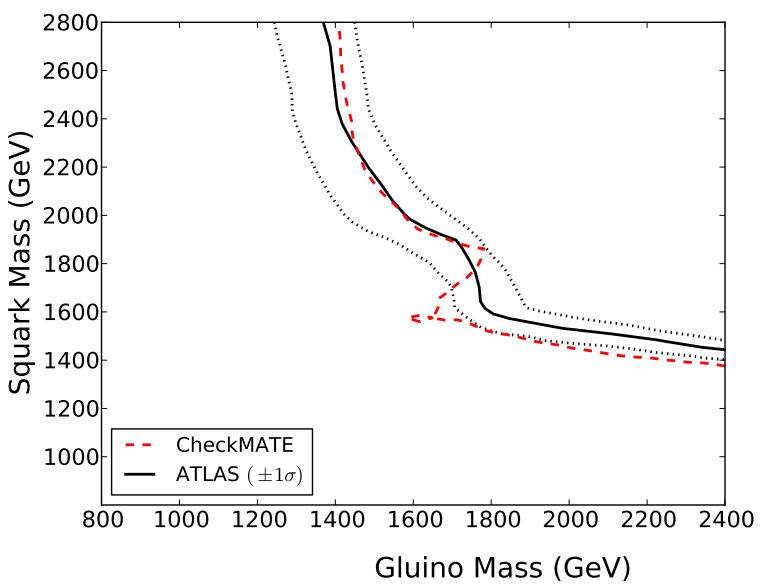

Squark Gluino Neutralino Model, $M_{\chi_{1}^{0}}=395 \mathrm{GeV}$

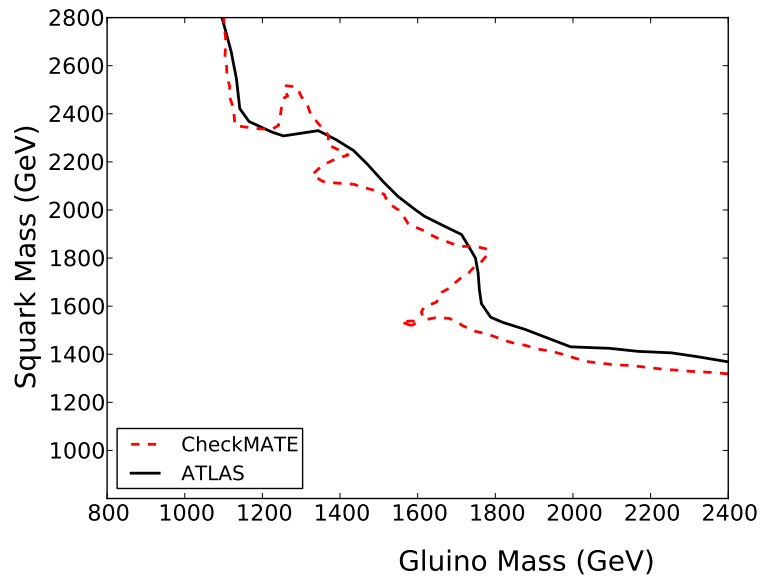

FIG. 24: Exclusion curve for the simplified model with only strong production of gluinos and first- and second-generation squarks. The lightest supersymmetric particle has mass, $\left(m_{\tilde{\chi}_{1}^{0}}\right)=0 \mathrm{GeV}$ (left) or $\left(m_{\tilde{\chi}_{1}^{0}}\right)=395 \mathrm{GeV}$ (right). Jumps in exclusion limit of Checkmate are due to the change of signal region.

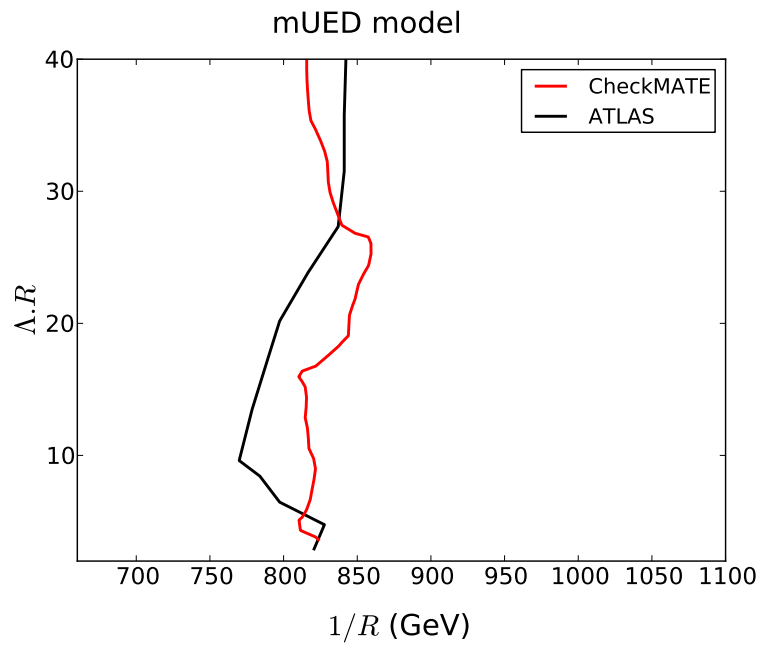

FIG. 25: Exclusion curve for the minimal Universal Extra Dimensions (mUED) model. Jumps in exclusion limit of Checkmate are due to the change of signal region. 


\section{6. atlas_conf_2013_049}

2 opposite-sign leptons, 0 jets $+E_{T}^{\text {miss }}, 32$

Energy: $8 \mathrm{TeV}$

Luminosity: $20.3 \mathrm{fb}^{-1}$

Validation notes:

- Validation has been performed versus all published cutflows.

- Jet veto conditions have been tightened by $25 \%$ compared to the corresponding stated ATLAS values. This was done to match cutflow values and account for pile-up effects that CheckmaTe cannot simulate.

- WARNING: For simplified models with chargino production and weak boson mediated decay, CheckMATE systematically underestimates signal region cutflows. Despite much effort, no reason for this discrepancy has been found. The corresponding setting is conservative since it will not lead to spurious model exclusion.

- No validation has yet been performed with parameter scans.

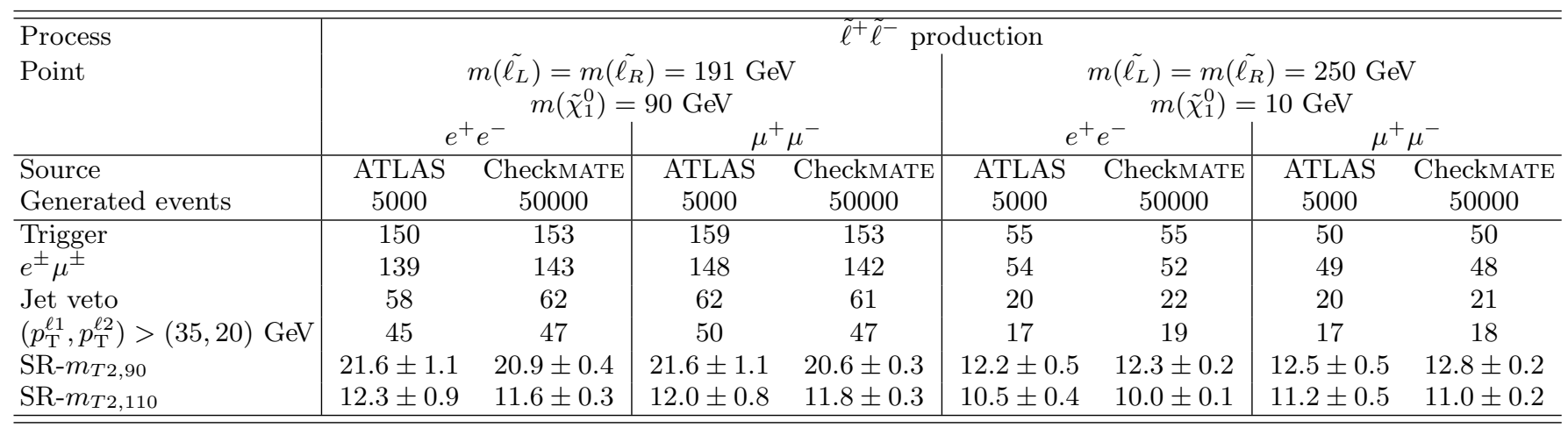

TABLE X: Shown are the number of events after each selection cut, normalised to $20.7 \mathrm{fb}^{-1}$. Final error is from Monte Carlo statistics for both ATLAS and CheckmaTE.

\begin{tabular}{|c|c|c|c|c|c|c|}
\hline \multirow{2}{*}{$\begin{array}{l}\text { Process } \\
\text { Point } \\
\\
\text { Source } \\
\text { Generated events }\end{array}$} & \multicolumn{6}{|c|}{$\begin{array}{c}\tilde{\chi}^{+} \tilde{\chi}^{-} \text {production, slepton decay } \\
m\left(\tilde{\chi}^{ \pm}\right)=350 \mathrm{GeV} \\
m\left(\tilde{\ell_{L}}\right)=m(\tilde{\nu})=175 \mathrm{GeV} \\
m\left(\tilde{\chi}_{1}^{0}\right)=0 \mathrm{GeV} \\
\mu^{+} \mu^{-}\end{array}$} \\
\hline & $\begin{array}{c}\text { ATLAS } \\
40000\end{array}$ & $\begin{array}{c}\text { CheckMATE } \\
50000\end{array}$ & $\begin{array}{c}\text { ATLAS } \\
40000\end{array}$ & $\begin{array}{c}\text { CheckMATE } \\
50000\end{array}$ & $\begin{array}{c}\text { ATLAS } \\
40000\end{array}$ & $\begin{array}{c}\text { CheckMATE } \\
50000\end{array}$ \\
\hline Trigger & 52 & 50 & 48 & 49 & 79 & 74 \\
\hline$\left(p_{\mathrm{T}}^{\ell 1}, p_{\mathrm{T}}^{\ell 2}\right)>(35,20) \mathrm{GeV}$ & 17 & 17 & 17 & 17 & 25 & 25 \\
\hline SR $-m_{T 2,90}$ & $11.7 \pm 0.4$ & $11.4 \pm 0.4$ & $10.5 \pm 0.4$ & $10.6 \pm 0.4$ & $16.6 \pm 0.5$ & $16.4 \pm 0.5$ \\
\hline $\mathrm{SR}-m_{T 2,110}$ & $9.5 \pm 0.4$ & $9.4 \pm 0.3$ & $8.7 \pm 0.4$ & $8.7 \pm 0.3$ & $14.0 \pm 0.4$ & $13.5 \pm 0.4$ \\
\hline
\end{tabular}

TABLE XI: Shown are the number of events after each selection cut, normalised to $20.7 \mathrm{fb}^{-1}$. Final error is from Monte Carlo statistics for both ATLAS and CheckmATE. 


\begin{tabular}{|c|c|c|c|c|c|c|}
\hline $\begin{array}{l}\text { Process } \\
\text { Point }\end{array}$ & \multicolumn{6}{|c|}{$\begin{array}{c}\tilde{\chi}^{+} \tilde{\chi}^{-} \text {production, slepton decay } \\
m\left(\tilde{\chi}^{ \pm}\right)=425 \mathrm{GeV} \\
m\left(\tilde{\ell_{L}}\right)=m(\tilde{\nu})=212.5 \mathrm{GeV} \\
m\left(\tilde{\chi}_{1}^{0}\right)=75 \mathrm{GeV}\end{array}$} \\
\hline $\begin{array}{l}\text { Source } \\
\text { Generated events }\end{array}$ & $\begin{array}{l}\text { ATLAS } \\
40000\end{array}$ & $\begin{array}{c}\text { Checkmate } \\
50000\end{array}$ & $\begin{array}{l}\text { ATLAS } \\
40000\end{array}$ & $\begin{array}{c}\text { Checkmate } \\
50000\end{array}$ & $\begin{array}{l}\text { ATLAS } \\
40000\end{array}$ & $\begin{array}{c}\text { CheckMATE } \\
50000\end{array}$ \\
\hline Trigger & 20 & 21 & 20 & 20 & 31 & 30 \\
\hline$e^{ \pm} \mu^{ \pm}$ & 19 & 20 & 19 & 19 & 29 & 28 \\
\hline Jet veto & 7 & 8 & 7 & 8 & 11 & 11 \\
\hline$\left(p_{\mathrm{T}}^{\ell 1}, p_{\mathrm{T}}^{\ell 2}\right)>(35,20) \mathrm{GeV}$ & 6 & 7 & 6 & 7 & 9 & 10 \\
\hline SR- $m_{T 2,90}$ & $4.3 \pm 0.2$ & $4.9 \pm 0.2$ & $4.4 \pm 0.2$ & $4.6 \pm 0.1$ & $6.7 \pm 0.2$ & $7.0 \pm 0.2$ \\
\hline SR- $m_{T 2,110}$ & $3.7 \pm 0.1$ & $4.3 \pm 0.1$ & $3.8 \pm 0.1$ & $3.9 \pm 0.1$ & $5.7 \pm 0.2$ & $5.9 \pm 0.2$ \\
\hline
\end{tabular}

TABLE XII: Shown are the number of events after each selection cut, normalised to $20.7 \mathrm{fb}^{-1}$. Final error is from Monte Carlo statistics for both ATLAS and Checkmate.

\begin{tabular}{|c|c|c|c|c|c|c|}
\hline $\begin{array}{l}\text { Process } \\
\text { Point }\end{array}$ & $\begin{array}{r}m\left(\tilde{\chi}_{1}^{ \pm}\right) \\
m\left(\tilde{\chi}_{1}^{0}\right.\end{array}$ & $\begin{array}{l}00 \mathrm{GeV} \\
0 \mathrm{GeV}\end{array}$ & $\begin{array}{r}{ }^{+} \tilde{\chi}^{-} \operatorname{prod} 1 \\
m\left(\tilde{\chi}_{1}^{ \pm}\right. \\
m\left(\tilde{\chi}_{1}^{0}\right.\end{array}$ & $\begin{array}{l}1, W W \text { decay } \\
40 \mathrm{GeV} \\
0 \mathrm{GeV}\end{array}$ & $\begin{array}{r}m\left(\tilde{\chi}_{1}^{ \pm}\right. \\
m(\tilde{\chi}\end{array}$ & $\begin{array}{l}00 \mathrm{GeV} \\
0 \mathrm{GeV}\end{array}$ \\
\hline $\begin{array}{l}\text { Source } \\
\text { Generated events }\end{array}$ & $\begin{array}{l}\text { ATLAS } \\
20000\end{array}$ & $\begin{array}{c}\text { CheckMATE } \\
50000\end{array}$ & $\begin{array}{l}\text { ATLAS } \\
20000\end{array}$ & $\begin{array}{c}\text { CheckMATE } \\
50000\end{array}$ & $\begin{array}{l}\text { ATLAS } \\
20000\end{array}$ & $\begin{array}{c}\text { CheckMATE } \\
50000\end{array}$ \\
\hline No Cuts & 11003 & - & 3393 & - & 749 & - \\
\hline All Cleaning * & 10691 & 10673 & 3299 & 3289 & 732 & 727 \\
\hline Two signal leptons & 3178 & 2610 & 1060 & 898 & 261 & 218 \\
\hline Trigger & 2559 & 1977 & 872 & 684 & 214 & 167 \\
\hline$e^{ \pm} \mu^{ \pm}$ & 861 & 803 & 296 & 288 & 71 & 69 \\
\hline Jet veto & 443 & 437 & 139 & 152 & 31 & 34 \\
\hline$\left(p_{\mathrm{T}}^{\ell 1}, p_{\mathrm{T}}^{\ell 2}\right)>(35,20) \mathrm{GeV}$ & 310 & 302 & 103 & 111 & 25 & 27 \\
\hline SR-WWa & $31.5 \pm 4.1$ & $21.6 \pm 2.4$ & - & - & - & - \\
\hline SR-WWb & - & - & $8.2 \pm 1.2$ & $4.5 \pm 0.6$ & - & - \\
\hline SR-WWc & - & - & - & - & $3.3 \pm 0.4$ & $2.6 \pm 0.2$ \\
\hline
\end{tabular}

TABLE XIII: Shown are the number of events after each selection cut, normalised to $20.7 \mathrm{fb}^{-1}$. Final error is from Monte Carlo statistics for both ATLAS and CheckmaTE. ${ }^{*}$ No cleaning cuts are performed by CheckMATE, instead a flat efficiency factor is applied to simulate this effect. 


\section{7. $\quad$ atlas_conf_2013_061}

Search for strong production with at least three $b$-jets $+E_{T}^{\text {miss }}$, 33 .

Energy: $8 \mathrm{TeV}$

Luminosity: $20.1 \mathrm{fb}^{-1}$

Validation notes:

- Validation has been performed versus all published cutflows.

- B-tagging efficiency was reduced by $3 \%$ compared with the nominal value in the experimental paper to better agree with cutflow data.

- No validation has yet been performed with parameter scans.

\begin{tabular}{|c|c|c|c|c|c|c|}
\hline $\begin{array}{l}\text { Process } \\
\text { Point } \\
\text { Channel }\end{array}$ & \multicolumn{6}{|c|}{$\begin{array}{c}p p \rightarrow \tilde{g} \tilde{g}, \tilde{g} \rightarrow b \bar{b} \tilde{\chi}_{1}^{0} \\
m(\tilde{g})=1300 \mathrm{GeV}, m\left(\tilde{\chi}_{1}^{0}\right)=100 \mathrm{GeV} \\
\text { 0 Lepton, 4 Jets }\end{array}$} \\
\hline Source & \multicolumn{3}{|c|}{ ATLAS } & \multicolumn{3}{|c|}{ CheckMATE } \\
\hline No selection & \multicolumn{3}{|c|}{$100 \%$} & \multicolumn{3}{|c|}{$-\%$} \\
\hline Jet and Event cleaning $*$ & \multicolumn{3}{|c|}{$98.2 \%$} & \multicolumn{3}{|c|}{$-\%$} \\
\hline Cosmic muon rejection $*$ & \multicolumn{3}{|c|}{$98.2 \%$} & \multicolumn{3}{|c|}{$-\%$} \\
\hline$\geq 4$ jets $\left(p_{\mathrm{T}}>30 \mathrm{GeV}\right)$ & \multicolumn{3}{|c|}{$95.4 \%$} & \multicolumn{3}{|c|}{$94.6 \%$} \\
\hline 1st jet $p_{\mathrm{T}}>90 \mathrm{GeV}$ & \multicolumn{3}{|c|}{$95.4 \%$} & \multicolumn{3}{|c|}{$94.6 \%$} \\
\hline $\mathbb{E}_{T}>150 \mathrm{GeV}$ & \multicolumn{3}{|c|}{$88.7 \%$} & \multicolumn{3}{|c|}{$88.0 \%$} \\
\hline Electron veto & \multicolumn{3}{|c|}{$88.7 \%$} & \multicolumn{3}{|c|}{$86.1 \%$} \\
\hline Muon veto & \multicolumn{3}{|c|}{$88.2 \%$} & \multicolumn{3}{|c|}{$85.7 \%$} \\
\hline$\Delta \phi_{\min }^{4 j}>0.5$ & \multicolumn{3}{|c|}{$58.5 \%$} & \multicolumn{3}{|c|}{$59.9 \%$} \\
\hline $\mathbb{E}_{T} / m_{\mathrm{eff}}^{4 j}>0.2$ & \multicolumn{3}{|c|}{$46.2 \%$} & \multicolumn{3}{|c|}{$48.7 \%$} \\
\hline Signal region cuts & \multicolumn{2}{|c|}{ SR-0l-4j-A } & \multicolumn{2}{|c|}{ SR-0l-4j-B } & \multicolumn{2}{|c|}{ SR-0l-4j-C } \\
\hline Source & ATLAS & Checkmate & ATLAS & Checkmate & ATLAS & CheckMATE \\
\hline$\geq 4 j, p_{\mathrm{T}}>30,50,50$ & $46.2 \%$ & $48.7 \%$ & $42.8 \%$ & $45.3 \%$ & $42.8 \%$ & $45.3 \%$ \\
\hline$\geq 3 b, p_{\mathrm{T}}>30,50,50$ & $20.5 \%$ & $20.0 \%$ & $17.9 \%$ & $17.5 \%$ & $17.9 \%$ & $17.4 \%$ \\
\hline $\bar{E}_{T}>200,350,250$ & $20.5 \%$ & $19.9 \%$ & $16.2 \%$ & $15.7 \%$ & $17.4 \%$ & $17.1 \%$ \\
\hline$m_{\mathrm{eff}}^{\text {incl }}>1000,1100,1300$ & $20.3 \%$ & $19.7 \%$ & $15.9 \pm 0.1 \%$ & $15.6 \pm 0.2 \%$ & $15.9 \pm 0.1 \%$ & $16.1 \pm 0.2 \%$ \\
\hline $\mathbb{E}_{T} / \sqrt{H_{T}^{4 j}}>16,0,0$ & $10.8 \pm 0.1 \%$ & $10.9 \pm 0.1 \%$ & - & - & - & - \\
\hline
\end{tabular}

TABLE XIV: The cutflow is given as an absolute efficiency in $\%$ for each step of event selection. Final error displayed on signal regions is due to finite Monte Carlo statistics. ${ }^{*}$ No cosmic muon rejection or event cleaning is performed by CheckMATE, instead a flat efficiency factor is included. 


\begin{tabular}{|c|c|c|c|c|c|c|}
\hline $\begin{array}{l}\text { Process } \\
\text { Point } \\
\text { Channel }\end{array}$ & \multicolumn{6}{|c|}{$\begin{array}{c}p p \rightarrow \tilde{g} \tilde{g}, \tilde{g} \rightarrow t \bar{t} \tilde{\chi}_{1}^{0} \\
m(\tilde{g})=1300 \mathrm{GeV}, m\left(\tilde{\chi}_{1}^{0}\right)=100 \mathrm{GeV} \\
\text { 0 Lepton, } 7 \text { Jets }\end{array}$} \\
\hline Source & \multicolumn{3}{|c|}{ ATLAS } & \multicolumn{3}{|c|}{ CheckMATE } \\
\hline No selection & \multicolumn{3}{|c|}{$100 \%$} & \multicolumn{3}{|c|}{$-\%$} \\
\hline Jet and Event cleaning $*$ & \multicolumn{3}{|c|}{$98.4 \%$} & \multicolumn{3}{|c|}{$-\%$} \\
\hline Cosmic muon rejection $*$ & \multicolumn{3}{|c|}{$97.2 \%$} & \multicolumn{3}{|c|}{$-\%$} \\
\hline$\geq 4$ jets $\left(p_{\mathrm{T}}>30 \mathrm{GeV}\right)$ & \multicolumn{3}{|c|}{$96.9 \%$} & \multicolumn{3}{|c|}{$97.6 \%$} \\
\hline 1st jet $p_{\mathrm{T}}>90 \mathrm{GeV}$ & \multicolumn{3}{|c|}{$96.9 \%$} & \multicolumn{3}{|c|}{$97.5 \%$} \\
\hline $\mathbb{E}_{T}>150 \mathrm{GeV}$ & \multicolumn{3}{|c|}{$88.3 \%$} & \multicolumn{3}{|c|}{$87.7 \%$} \\
\hline Electron veto & \multicolumn{3}{|c|}{$59.7 \%$} & \multicolumn{3}{|c|}{$60.2 \%$} \\
\hline Muon veto & \multicolumn{3}{|c|}{$41.8 \%$} & \multicolumn{3}{|c|}{$40.7 \%$} \\
\hline$\Delta \phi_{\min }^{4 j}>0.5$ & \multicolumn{3}{|c|}{$30.0 \%$} & \multicolumn{3}{|c|}{$29.6 \%$} \\
\hline $\mathbb{E}_{T} / m_{\mathrm{eff}}^{4 j}>0.2$ & \multicolumn{3}{|c|}{$25.9 \%$} & \multicolumn{3}{|c|}{$25.4 \%$} \\
\hline$\geq 7$ jets $\left(p_{\mathrm{T}}>30 \mathrm{GeV}\right)$ & \multicolumn{3}{|c|}{$24.6 \%$} & \multicolumn{3}{|c|}{$24.1 \%$} \\
\hline$\geq 3 b$-jets $\left(p_{\mathrm{T}}>30 \mathrm{GeV}\right)$ & \multicolumn{3}{|c|}{$11.5 \%$} & \multicolumn{3}{|c|}{$11.4 \%$} \\
\hline Signal region cuts & \multicolumn{2}{|c|}{ SR-0l-7j-A } & \multicolumn{2}{|c|}{ SR-0l-7j-B } & \multicolumn{2}{|c|}{ SR-0l-7j-C } \\
\hline Source & ATLAS & Checkmate & ATLAS & Checkmate & ATLAS & Checkmate \\
\hline $\mathbb{E}_{T}>200,350,250$ & $11.3 \%$ & $11.3 \%$ & $9.2 \%$ & $8.9 \%$ & $10.8 \%$ & $10.8 \%$ \\
\hline$m_{\mathrm{eff}}^{i \text { incl }}>1000,1000,1500$ & $11.3 \pm 0.1 \%$ & $11.2 \pm 0.1 \%$ & $9.2 \pm 0.1 \%$ & $8.9 \pm 0.1 \%$ & $9.5 \pm 0.1 \%$ & $9.2 \pm 0.1 \%$ \\
\hline
\end{tabular}

TABLE XV: The cutflow is given as an absolute efficiency in $\%$ for each step of event selection. Final error displayed on signal regions is due to finite Monte Carlo statistics. ${ }^{*}$ No cosmic muon rejection or event cleaning is performed by CheckMATE, instead a flat efficiency factor is included.

\begin{tabular}{|c|c|c|c|c|c|c|}
\hline $\begin{array}{l}\text { Process } \\
\text { Point }\end{array}$ & \multicolumn{6}{|c|}{$\begin{array}{c}p p \rightarrow \tilde{g} \tilde{g}, \tilde{g} \rightarrow t \bar{t} \tilde{\chi}_{1}^{0} \\
m(\tilde{g})=1300 \mathrm{GeV}, m\left(\tilde{\chi}_{1}^{0}\right)=100 \mathrm{GeV}\end{array}$} \\
\hline Channel & \multirow{2}{*}{\multicolumn{4}{|c|}{1 Lepton }} & \multirow{2}{*}{\multicolumn{2}{|c|}{ CheckMATE }} \\
\hline Source & & & & & & \\
\hline No selection & \multicolumn{3}{|c|}{$100 \%$} & \multicolumn{3}{|c|}{$-\%$} \\
\hline Jet and Event cleaning * & \multicolumn{3}{|c|}{$98.4 \%$} & \multicolumn{3}{|c|}{$-\%$} \\
\hline Cosmic muon rejection $*$ & \multicolumn{3}{|c|}{$97.2 \%$} & \multicolumn{3}{|c|}{$-\%$} \\
\hline$\geq 4$ jets $\left(p_{\mathrm{T}}>30 \mathrm{GeV}\right)$ & \multicolumn{3}{|c|}{$96.9 \%$} & \multicolumn{3}{|c|}{$97.6 \%$} \\
\hline 1st jet $p_{\mathrm{T}}>90 \mathrm{GeV}$ & \multicolumn{3}{|c|}{$96.8 \%$} & \multicolumn{3}{|c|}{$97.5 \%$} \\
\hline $\mathbb{E}_{T}>150 \mathrm{GeV}$ & \multicolumn{3}{|c|}{$88.3 \%$} & \multicolumn{3}{|c|}{$87.5 \%$} \\
\hline$\geq 1$ signal lepton & \multicolumn{3}{|c|}{$37.0 \%$} & \multicolumn{3}{|c|}{$40.6 \%$} \\
\hline$\geq 6$ jets $\left(p_{\mathrm{T}}>30 \mathrm{GeV}\right)$ & \multicolumn{3}{|c|}{$33.8 \%$} & \multicolumn{3}{|c|}{$36.7 \%$} \\
\hline$\geq 3 b$-jets $\left(p_{\mathrm{T}}>30 \mathrm{GeV}\right)$ & \multicolumn{3}{|c|}{$14.3 \%$} & \multicolumn{3}{|c|}{$16.1 \%$} \\
\hline Signal region cuts & \multicolumn{2}{|c|}{ SR-1l-6j-A } & \multicolumn{2}{|c|}{ SR-1l-6j-B } & \multicolumn{2}{|c|}{ SR-1l-6j-C } \\
\hline Source & ATLAS & Checkmate & ATLAS & Checkmate & ATLAS & Checkmate \\
\hline$m_{T}>140,140,160 \mathrm{GeV}$ & $11.3 \%$ & $12.0 \%$ & $11.3 \%$ & $12.0 \%$ & $10.7 \%$ & $11.3 \%$ \\
\hline $\mathbb{E}_{T}>175,225,275 \mathrm{GeV}$ & $10.9 \%$ & $11.6 \%$ & $10.0 \%$ & $10.7 \%$ & $8.8 \%$ & $9.3 \%$ \\
\hline $\mathbb{E}_{T} / \sqrt{H_{T}}>5 \mathrm{GeV}^{1 / 2}$ & $10.8 \%$ & $11.3 \%$ & $10.0 \%$ & $10.6 \%$ & $8.8 \%$ & $9.3 \%$ \\
\hline$m_{\mathrm{eff}}>700,800,900 \mathrm{GeV}$ & $10.8 \pm 0.1 \%$ & $11.3 \pm 0.2 \%$ & $10.0 \pm 0.1 \%$ & $10.6 \pm 0.2 \%$ & $8.8 \pm 0.1 \%$ & $9.2 \pm 0.1 \%$ \\
\hline
\end{tabular}

TABLE XVI: The cutflow is given as an absolute efficiency in \% for each step of event selection. Final error displayed on signal regions is due to finite Monte Carlo statistics. * No cosmic muon rejection or event cleaning is performed by CheckMATE, instead a flat efficiency factor is included. 


\section{8. $\quad$ atlas_conf_2013_089}

Strongly produced SUSY with two leptons (razor), 35.

Energy: $8 \mathrm{TeV}$

Luminosity: $20.3 \mathrm{fb}^{-1}$

Validation notes:

- Validation has been performed versus all published cutflows.

- WARNING: Discrepancies exist in the final signal regions between ATLAS and Checkmate. However, we do not believe this shows a systematic failing of CheckMATE. First of all, the statistics from ATLAS are very small and it is therefore hard to draw a definitive conclusion. Moreover, after the trigger has been performed, no cut has an obvious flavour dependence. Consequently it is expected (and confirmed by ATLAS) that the $e \mu$ channels should contain the largest number of events.

- We see a difference in the exclusion for low squark masses and a heavier LSP. We believe this is due to different settings in the Monte Carlo parton shower (Pythia 6) that gives a harder initial state radiation distribution.

\begin{tabular}{|c|c|c|c|c|c|c|}
\hline $\begin{array}{l}\text { Process } \\
\text { Point }\end{array}$ & \multicolumn{6}{|c|}{$\begin{aligned} & \tilde{g} \tilde{g} \text { production, } \tilde{g} \rightarrow q q \tilde{\chi}_{1}^{ \pm}, \tilde{\chi}_{1}^{ \pm} \rightarrow W \tilde{\chi}_{1}^{0} \\
m(\tilde{g})= & 800 \mathrm{GeV}, m\left(\tilde{\chi}_{1}^{ \pm}\right)=460 \mathrm{GeV}, m\left(\tilde{\chi}_{1}^{0}\right)=60 \mathrm{GeV}\end{aligned}$} \\
\hline Source & \multirow{2}{*}{\multicolumn{3}{|c|}{$\begin{array}{l}\text { ATLAS } \\
59999\end{array}$}} & \multirow{2}{*}{\multicolumn{3}{|c|}{$\begin{array}{c}\text { CheckMATE } \\
59999\end{array}$}} \\
\hline No Cuts & & & & & & \\
\hline 2 baseline leptons & \multicolumn{3}{|c|}{2073} & \multicolumn{3}{|c|}{1880} \\
\hline Passes trigger & \multicolumn{3}{|c|}{1670} & \multicolumn{3}{|c|}{1426} \\
\hline$m_{\ell \ell}>20 \mathrm{GeV}$ & \multicolumn{3}{|c|}{1639} & \multicolumn{3}{|c|}{1408} \\
\hline Lepton flavour & \multicolumn{2}{|c|}{$e e$} & \multicolumn{2}{|c|}{$\mu \mu$} & \multicolumn{2}{|c|}{$e \mu$} \\
\hline Source & ATLAS & Checkmate & ATLAS & Checkmate & ATLAS & CheckMATE \\
\hline Lepton separation & 576 & 420 & 397 & 397 & 666 & 590 \\
\hline Signal leptons & 443 & 377 & 373 & 397 & 549 & 561 \\
\hline Trigger + dilepton & 429 & 377 & 358 & 397 & 517 & 561 \\
\hline$>2$ jets, $b$-veto & 341 & 320 & 297 & 350 & 424 & 480 \\
\hline$Z$-veto & 319 & 293 & 276 & 321 & 424 & 480 \\
\hline$R>0.35$ & 139 & 155 & 137 & 162 & 195 & 252 \\
\hline$M_{R}^{\prime}>800 \mathrm{GeV}$ & $53 \pm 7$ & $55 \pm 2$ & $50 \pm 7$ & $60 \pm 3$ & $63 \pm 8$ & $92 \pm 3$ \\
\hline$<3$ jets, $b$-veto & 53 & 44 & 40 & 34 & 54 & 56 \\
\hline$Z$-veto & 51 & 41 & 36 & 31 & 54 & 56 \\
\hline$R>0.5$ & 16 & 14 & 19 & 10 & 12 & 21 \\
\hline$M_{R}^{\prime}>400 \mathrm{GeV}$ & $14 \pm 4$ & $13 \pm 1$ & $19 \pm 4$ & $10 \pm 1$ & $10 \pm 3$ & $19 \pm 1$ \\
\hline
\end{tabular}

TABLE XVII: The ATLAS column shows the number of Monte Carlo events after each selection cut. The Checkmate cutflow is normalised to number of events before any selection cuts are applied. The final error is from Monte Carlo statistics for both ATLAS and CheckMATE. 


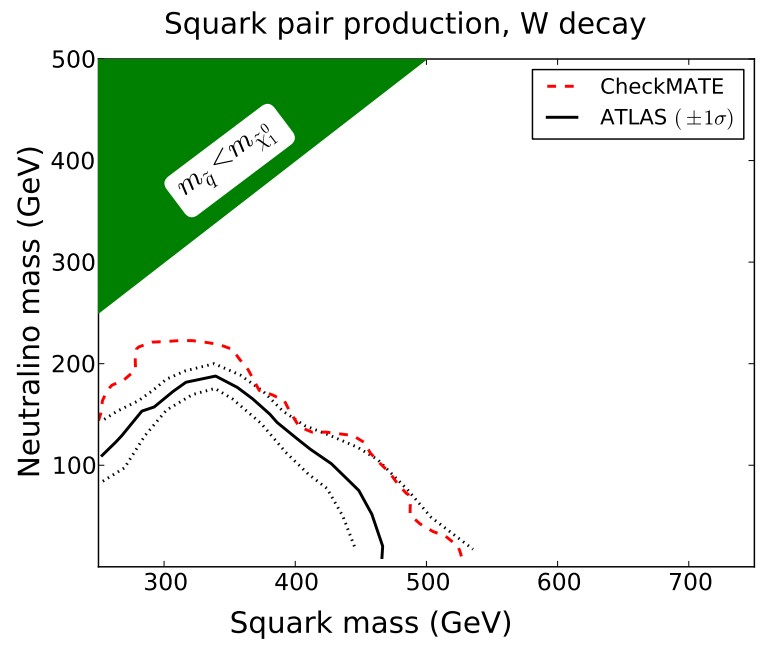

FIG. 26: Exclusion curve for a simplified model with squark production followed by decay $\tilde{q} \rightarrow \tilde{\chi}_{1}^{ \pm} q, \tilde{\chi}_{1}^{ \pm} \rightarrow W \tilde{\chi}_{1}^{0}$.

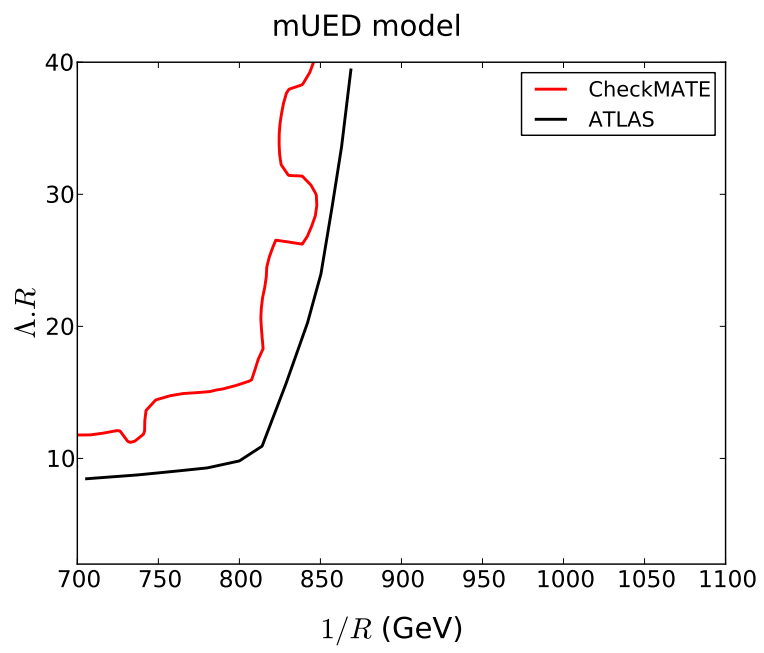

FIG. 27: Exclusion curve for the minimal Universal Extra Dimensions (mUED) model. Jumps in exclusion limit of Checkmate are due to the change of signal region. 


\section{9. $\quad c m s \_1303 \_2985$}

Hadronic $\alpha_{T}$ and $b$-jet multiplicity, [37]

Energy: $8 \mathrm{TeV}$

Luminosity: $11.7 \mathrm{fb}^{-1}$

Validation notes:

- No cut flow is provided by CMS, validation is performed with signal region distributions and parameter scans.

- The SM background distributions has been taken from the CMS note.

- The ATLAS $b$-tagging has been used.

- Jumps in limit in Checkmate parameter scans are due to the single signal region limit setting procedure used (see Section IB).
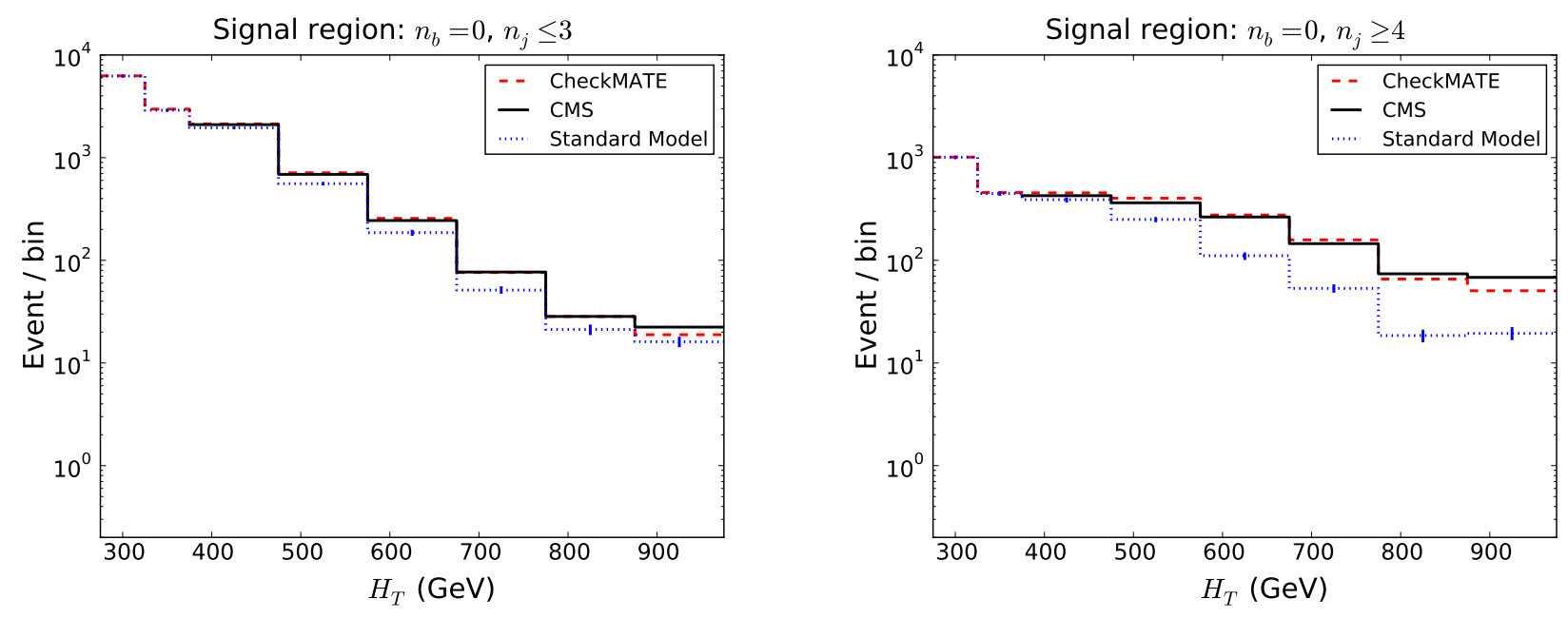

FIG. 28: Distributions in $H_{T}$ for different signal models. Left: $p p \rightarrow \tilde{q} \tilde{q}, \tilde{q} \rightarrow q \tilde{\chi}_{1}^{0},\left(m_{\tilde{q}}=600 \mathrm{GeV}, m_{\tilde{\chi}_{1}^{0}}=250 \mathrm{GeV}\right)$. Right: $p p \rightarrow \tilde{g} \tilde{g}, \tilde{g} \rightarrow q \bar{q} \tilde{\chi}_{1}^{0},\left(m_{\tilde{g}}=700 \mathrm{GeV}, m_{\tilde{\chi}_{1}^{0}}=300 \mathrm{GeV}\right)$ 

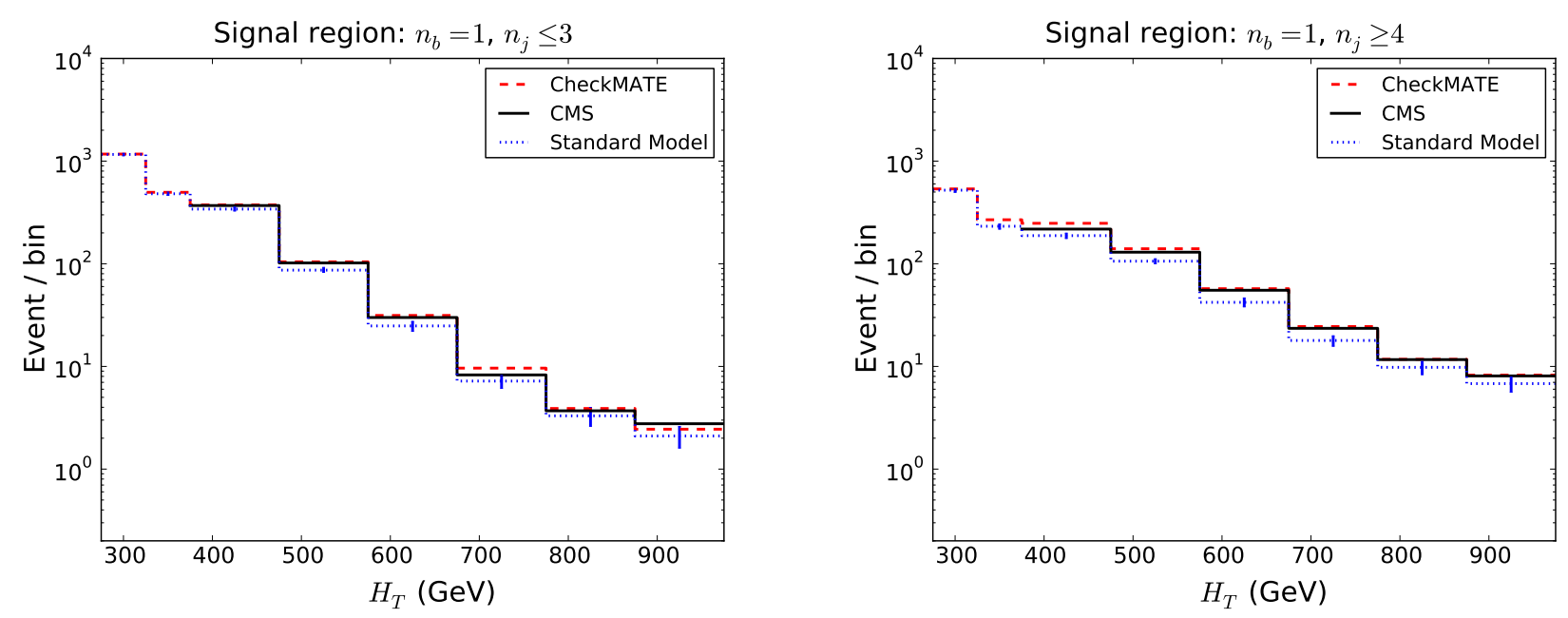

FIG. 29: Distributions in $H_{T}$ for different signal models. Left: $p p \rightarrow \tilde{b} \tilde{b}, \tilde{b} \rightarrow b \tilde{\chi}_{1}^{0},\left(m_{\tilde{b}}=500 \mathrm{GeV}, m_{\tilde{\chi}_{1}^{0}}=150 \mathrm{GeV}\right)$. Right: $p p \rightarrow \tilde{t} \tilde{t}, \tilde{t} \rightarrow t \tilde{\chi}_{1}^{0},\left(m_{\tilde{t}}=400 \mathrm{GeV}, m_{\tilde{\chi}_{1}^{0}}=0 \mathrm{GeV}\right)$
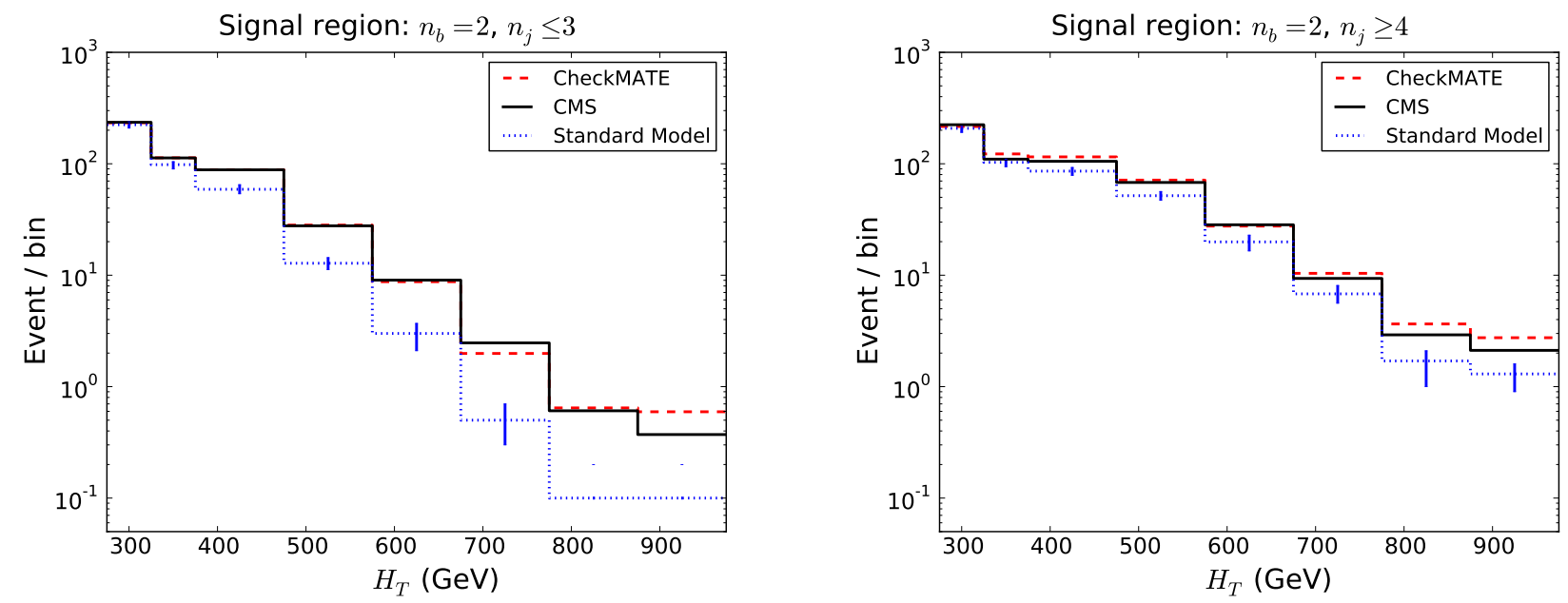

FIG. 30: Distributions in $H_{T}$ for different signal models. Left: $p p \rightarrow \tilde{b} \tilde{b}, \tilde{b} \rightarrow b \tilde{\chi}_{1}^{0},\left(m_{\tilde{b}}=500 \mathrm{GeV}, m_{\tilde{\chi}_{1}^{0}}=150 \mathrm{GeV}\right)$. Right: $p p \rightarrow \tilde{t} \tilde{t}, \tilde{t} \rightarrow t \tilde{\chi}_{1}^{0},\left(m_{\tilde{t}}=400 \mathrm{GeV}, m_{\tilde{\chi}_{1}^{0}}=0 \mathrm{GeV}\right)$ 

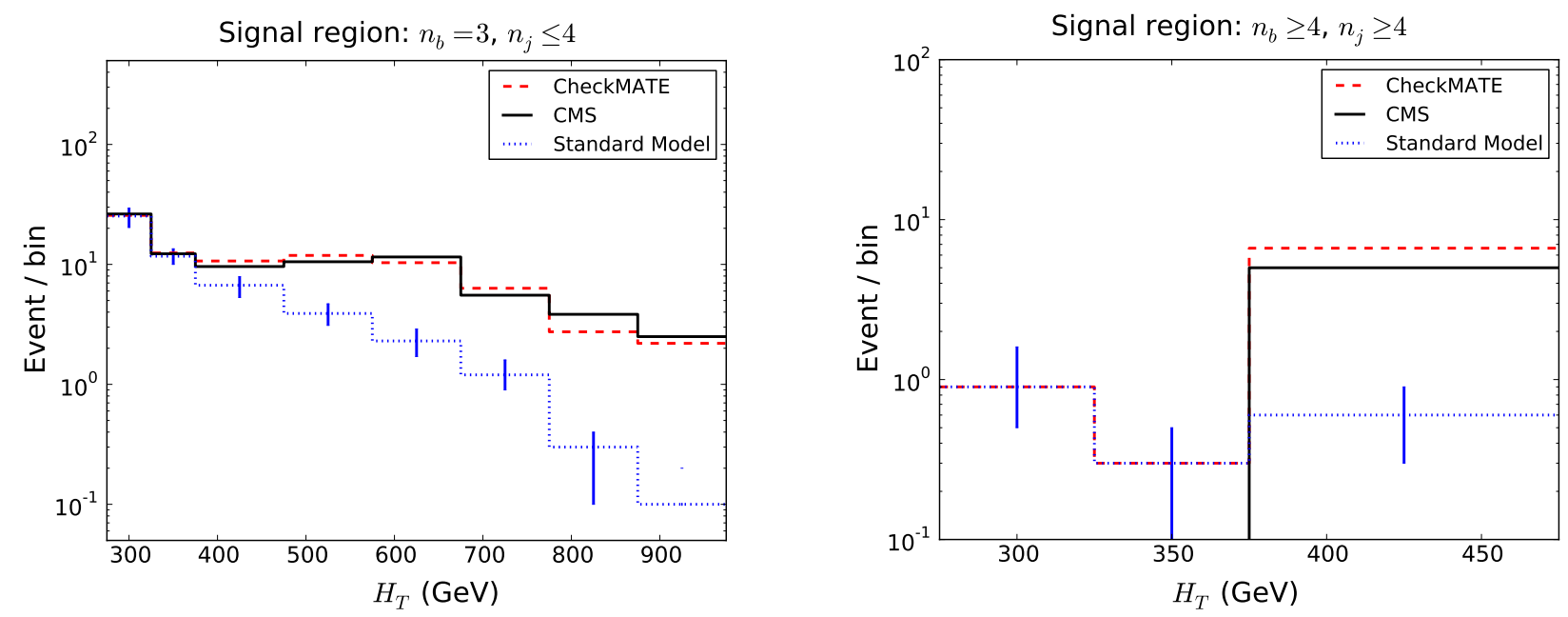

FIG. 31: Distributions in $H_{T}$ for different signal models. Left: $p p \rightarrow \tilde{g} \tilde{g}, \tilde{g} \rightarrow b \bar{b} \tilde{\chi}_{1}^{0},\left(m_{\tilde{g}}=900 \mathrm{GeV}, m_{\tilde{\chi}_{1}^{0}}=500 \mathrm{GeV}\right)$. Right: $p p \rightarrow \tilde{g} \tilde{g}, \tilde{g} \rightarrow t \bar{t} \tilde{\chi}_{1}^{0},\left(m_{\tilde{g}}=850 \mathrm{GeV}, m_{\tilde{\chi}_{1}^{0}}=250 \mathrm{GeV}\right)$
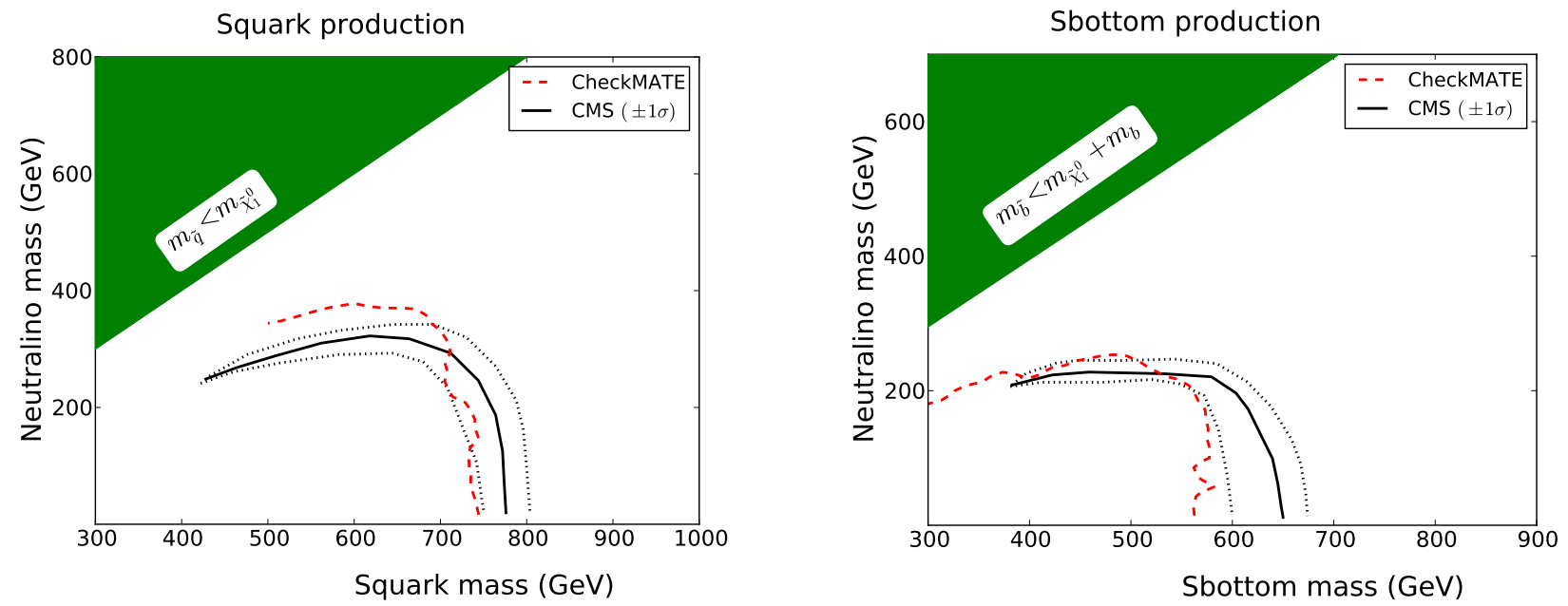

FIG. 32: Exclusion curve for a simplified model with only first and second generation squark production (left) and only bottom squark production (right). We see a difference in exclusion for first and second generation squark production with smaller squark masses and a heavier LSP. We believe this is due to different settings in the Monte Carlo parton shower (Pythia 6) that gives a harder initial state radiation distribution. 

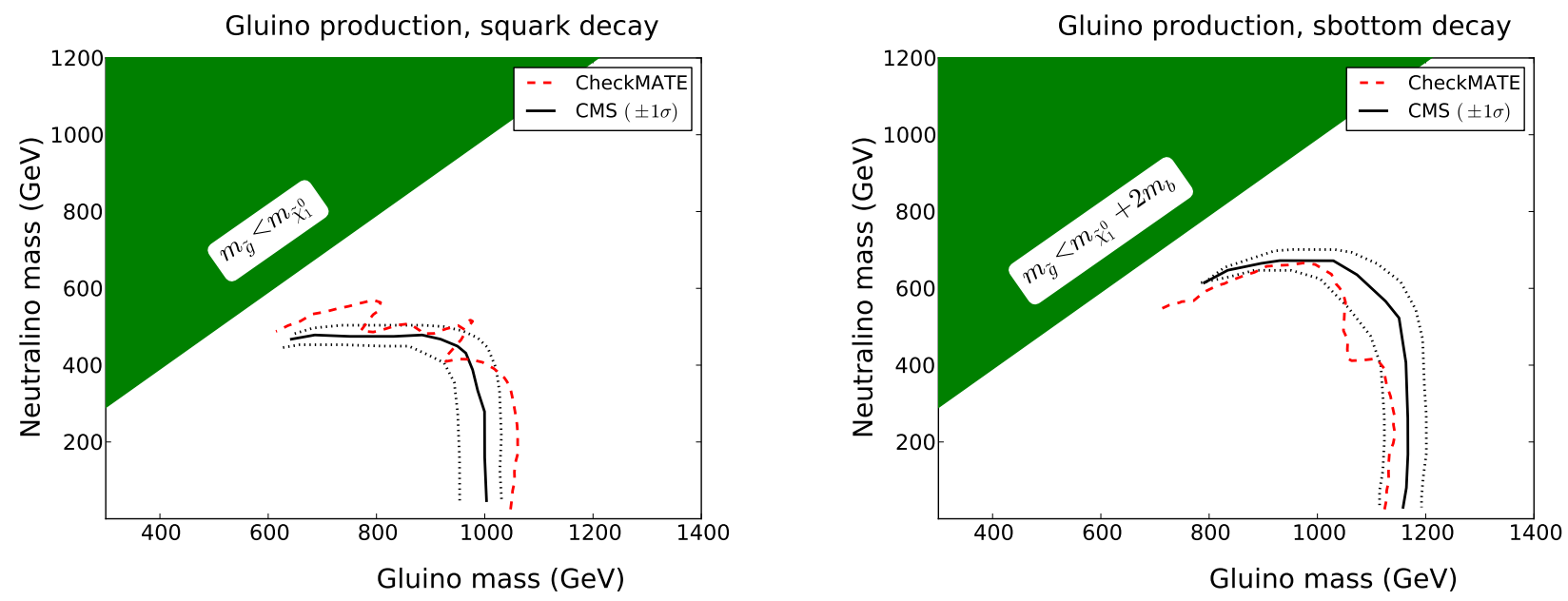

FIG. 33: Exclusion curve for a simplified model with gluino production followed by decay into a $q \bar{q} \tilde{\chi}_{1}^{0}$ final state (left) or $b \bar{b} \tilde{\chi}_{1}^{0}$ final state (right).

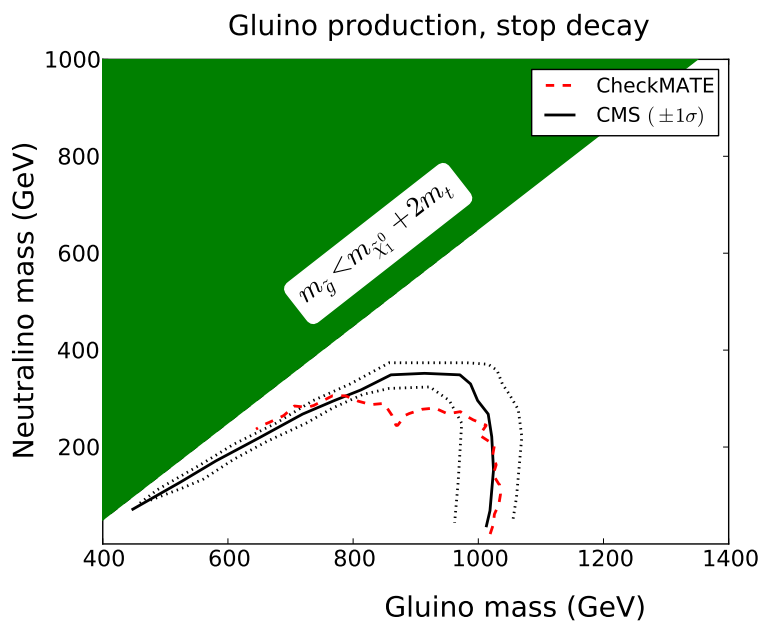

FIG. 34: Exclusion curve for a simplified model with gluino production followed by decay into a $t \bar{t} \tilde{\chi}_{1}^{0}$ final state. 
[1] J. de Favereau, C. Delaere, P. Demin, A. Giammanco, V. Lematre, et al., "DELPHES 3, A modular framework for fast simulation of a generic collider experiment," 2013.

[2] M. Cacciari, G. P. Salam, and G. Soyez, "FastJet User Manual," Eur.Phys.J., vol. C72, p. 1896, 2012.

[3] M. Cacciari and G. P. Salam, "Dispelling the $N^{3}$ myth for the $k_{t}$ jet-finder," Phys.Lett., vol. B641, pp. 57-61, 2006.

[4] M. Cacciari, G. P. Salam, and G. Soyez, "The Anti-k(t) jet clustering algorithm," JHEP, vol. 0804, p. $063,2008$.

[5] A. L. Read, "Presentation of search results: the cl's technique," Journal of Physics G: Nuclear and Particle Physics, vol. 28 , no. 10, p. 2693, 2002.

[6] C. Lester and D. Summers, "Measuring masses of semiinvisibly decaying particles pair produced at hadron colliders," Phys.Lett., vol. B463, pp. 99-103, 1999.

[7] A. Barr, C. Lester, and P. Stephens, "m(T2): The Truth behind the glamour," J.Phys., vol. G29, pp. 2343-2363, 2003.

[8] H.-C. Cheng and Z. Han, "Minimal Kinematic Constraints and m(T2)," JHEP, vol. 0812, p. $063,2008$.

[9] H. E. Haber and G. L. Kane, "The Search for Supersymmetry: Probing Physics Beyond the Standard Model," Phys.Rept., vol. 117 , pp. $75-263,1985$.

[10] H. P. Nilles, "Supersymmetry, Supergravity and Particle Physics," Phys.Rept., vol. 110, pp. 1-162, 1984.

[11] S. Weinberg, "Implications of Dynamical Symmetry Breaking," Phys.Rev., vol. D13, pp. 974-996, 1976.

[12] L. Susskind, "Dynamics of Spontaneous Symmetry Breaking in the Weinberg-Salam Theory," Phys.Rev., vol. D20, pp. 2619-2625, 1979.

[13] N. Arkani-Hamed, S. Dimopoulos, and G. Dvali, "The Hierarchy problem and new dimensions at a millimeter," Phys.Lett., vol. B429, pp. 263-272, 1998.

[14] L. Randall and R. Sundrum, "A Large mass hierarchy from a small extra dimension," Phys.Rev.Lett., vol. 83, pp. 3370-3373, 1999.

[15] D. Alves et al., "Simplified Models for LHC New Physics Searches," J.Phys., vol. G39, p. 105005, 2012.

[16] G. Bertone, D. Hooper, and J. Silk, "Particle dark matter: Evidence, candidates and constraints," Phys.Rept., vol. 405, pp. 279-390, 2005.

[17] M. Dobbs and J. B. Hansen, "The HepMC C++ Monte Carlo event record for High Energy Physics," Comput.Phys.Commun., vol. 134, pp. 41-46, 2001.

[18] I. Knowles, T. Sjostrand, A. Blondel, A. Boehrer, C. Buchanan, et al., "QCD event generators," 1995.

[19] T. Sjostrand, S. Mrenna, and P. Z. Skands, "PYTHIA 6.4 Physics and Manual," JHEP, vol. 0605, p. $026,2006$.

[20] T. Sjostrand, S. Mrenna, and P. Z. Skands, "A Brief Introduction to PYTHIA 8.1," Comput.Phys.Commun., vol. 178, pp. 852-867, 2008.

[21] M. Bahr, S. Gieseke, M. Gigg, D. Grellscheid, K. Hamilton, et al., "Herwig++ Physics and Manual," Eur.Phys.J., vol. C58, pp. 639-707, 2008.

[22] A. Belyaev, N. D. Christensen, and A. Pukhov, "CalcHEP 3.4 for collider physics within and beyond the Standard Model," Comput.Phys.Commun., vol. 184, pp. 1729-1769, 2013.

[23] J. Alwall, M. Herquet, F. Maltoni, O. Mattelaer, and T. Stelzer, "MadGraph 5 : Going Beyond," JHEP, vol. 1106, p. 128, 2011.

[24] T. Gleisberg, S. Hoeche, F. Krauss, M. Schonherr, S. Schumann, et al., "Event generation with SHERPA 1.1," JHEP, vol. 0902, p. 007, 2009.

[25] R. Brun and F. Rademakers, "ROOT: An object oriented data analysis framework," Nucl.Instrum.Meth., vol. A389, pp. 81-86, 1997.

[26] G. Aad et al., "Search for direct third-generation squark pair production in final states with missing transverse momentum and two b-jets in $\sqrt{s}=8 \mathrm{TeV}$ pp collisions with the ATLAS detector," 2013.

[27] "Search for supersymmetry at $\sqrt{s}=8$ tev in final states with jets, missing transverse momentum and one isolated lepton," Tech. Rep. ATLAS-CONF-2012-104, CERN, Geneva, Aug 2012.

[28] "Search for new phenomena in monojet plus missing transverse momentum final states using $10 \mathrm{fb}-1$ of pp collisions at sqrts=8 tev with the atlas detector at the lhc," Tech. Rep. ATLAS-CONF-2012-147, CERN, Geneva, Nov 2012.

[29] "Search for direct production of the top squark in the all-hadronic ttbar + etmiss final state in $21 \mathrm{fb}-1$ of p-pcollisions at sqrt(s)=8 tev with the atlas detector," Tech. Rep. ATLAS-CONF-2013-024, CERN, Geneva, Mar 2013.

[30] "Search for direct production of charginos and neutralinos in events with three leptons and missing transverse momentum in $21 \mathrm{fb}^{-1}$ of pp collisions at $\sqrt{s}=8$ tev with the atlas detector," Tech. Rep. ATLAS-CONF-2013-035, CERN, Geneva, Mar 2013.

[31] "Search for squarks and gluinos with the atlas detector in final states with jets and missing transverse momentum and $20.3 \mathrm{fb}^{-1}$ of $\sqrt{s}=8$ tev proton-proton collision data," Tech. Rep. ATLAS-CONF-2013-047, CERN, Geneva, May 2013.

[32] "Search for direct-slepton and direct-chargino production in final states with two opposite-sign leptons, missing transverse momentum and no jets in $20 / \mathrm{fb}$ of pp collisions at sqrt(s) = 8 tev with the atlas detector," Tech. Rep. ATLAS-CONF2013-049, CERN, Geneva, May 2013.

[33] "Search for strong production of supersymmetric particles in final states with missing transverse momentum and at least three b-jets using $20.1 \mathrm{fb} 1$ of pp collisions at sqrt(s) = 8 tev with the atlas detector.," Tech. Rep. ATLAS-CONF-2013-061, CERN, Geneva, Jun 2013.

[34] "Search for squarks and gluinos in events with isolated leptons, jets and missing transverse momentum at $\sqrt{s}=8$ tev with the atlas detector," Tech. Rep. ATLAS-CONF-2013-062, CERN, Geneva, Jun 2013. 
[35] "Search for strongly produced supersymmetric particles in decays with two leptons at $\sqrt{s}=8$ tev," Tech. Rep. ATLASCONF-2013-089, CERN, Geneva, Aug 2013.

[36] "Search for new physics in monojet events in pp collisions at sqrt(s)= 8 tev," Tech. Rep. CMS-PAS-EXO-12-048, CERN, Geneva, 2013.

[37] S. Chatrchyan et al., "Search for supersymmetry in hadronic final states with missing transverse energy using the variables $\alpha_{T}$ and b-quark multiplicity in pp collisions at $\sqrt{s}=8 \mathrm{TeV}, " 2013$.

[38] W. Beenakker, M. Kramer, T. Plehn, M. Spira, and P. Zerwas, "Stop production at hadron colliders," Nucl.Phys., vol. B515, pp. 3-14, 1998.

[39] A. Kulesza and L. Motyka, "Threshold resummation for squark-antisquark and gluino-pair production at the LHC," Phys.Rev.Lett., vol. 102, p. 111802, 2009.

[40] A. Kulesza and L. Motyka, "Soft gluon resummation for the production of gluino-gluino and squark-antisquark pairs at the LHC," Phys.Rev., vol. D80, p. 095004, 2009.

[41] W. Beenakker, S. Brensing, M. Kramer, A. Kulesza, E. Laenen, et al., "Soft-gluon resummation for squark and gluino hadroproduction," JHEP, vol. 0912, p. 041, 2009.

[42] W. Beenakker, S. Brensing, M. Kramer, A. Kulesza, E. Laenen, et al., "Supersymmetric top and bottom squark production at hadron colliders," JHEP, vol. 1008, p. 098, 2010.

[43] W. Beenakker, S. Brensing, M. Kramer, A. Kulesza, E. Laenen, et al., "Squark and Gluino Hadroproduction," Int.J.Mod.Phys., vol. A26, pp. 2637-2664, 2011.

[44] G. Aad et al., "Electron performance measurements with the ATLAS detector using the 2010 LHC proton-proton collision data," Eur.Phys.J., vol. C72, p. 1909, 2012.

[45] "Electron efficiency measurements for 2012 and 2011 data," Tech. Rep. ATL-COM-PHYS-2013-1287, CERN, Geneva, Sep 2013.

[46] "Muon reconstruction efficiency in reprocessed 2010 LHC proton-proton collision data recorded with the ATLAS detector," Tech. Rep. ATLAS-CONF-2011-063, CERN, Geneva, Apr 2011.

[47] A. Salvucci, "Measurement of muon momentum resolution of the ATLAS detector," in European Physical Journal Web of Conferences, vol. 28 of European Physical Journal Web of Conferences, p. 12039, June 2012.

[48] G. Aad et al., "Expected Performance of the ATLAS Experiment - Detector, Trigger and Physics," 2009.

[49] "Commissioning of the ATLAS high-performance b-tagging algorithms in the 7 TeV collision data," Tech. Rep. ATLASCONF-2011-102, CERN, Geneva, Jul 2011.

[50] " $b$-jet tagging calibration on $c$-jets containing $D^{*+}$ mesons," Tech. Rep. ATLAS-CONF-2012-039, CERN, Geneva, Mar 2012.

[51] "Measurement of the Mistag Rate with $5 \mathrm{fb}^{1}$ of Data Collected by the ATLAS Detector," Tech. Rep. ATLAS-CONF-2012040, CERN, Geneva, Mar 2012.

[52] "Measuring the b-tag efficiency in a top-pair sample with $4.7 \mathrm{fb}-1$ of data from the ATLAS detector," Tech. Rep. ATLASCONF-2012-097, CERN, Geneva, Jul 2012.

[53] "Performance of the Reconstruction and Identification of Hadronic Tau Decays with ATLAS," Tech. Rep. ATLAS-CONF2011-152, CERN, Geneva, Nov 2011.

[54] G. Giudice and R. Rattazzi, "Theories with gauge mediated supersymmetry breaking," Phys.Rept., vol. 322, pp. 419-499, 1999.

[55] J. Goodman, M. Ibe, A. Rajaraman, W. Shepherd, T. M. Tait, et al., "Constraints on Dark Matter from Colliders," Phys.Rev., vol. D82, p. 116010, 2010.

[56] H. K. Dreiner, M. Kramer, and J. Tattersall, "How low can SUSY go? Matching, monojets and compressed spectra," Europhys.Lett., vol. 99, p. 61001, 2012.

[57] H. Dreiner, M. Kramer, and J. Tattersall, "Exploring QCD uncertainties when setting limits on compressed supersymmetric spectra," Phys.Rev., vol. D87, p. 035006, 2013.

[58] C. Rogan, "Kinematical variables towards new dynamics at the LHC," 2010.

[59] G. Aad et al., "Measurement of the inclusive $W^{ \pm}$and Z/gamma cross sections in the electron and muon decay channels in $p p$ collisions at $\sqrt{s}=7 \mathrm{TeV}$ with the ATLAS detector," Phys.Rev., vol. D85, p. 072004, 2012.

[60] A. Alloul, N. D. Christensen, C. Degrande, C. Duhr, and B. Fuks, "FeynRules 2.0 - A complete toolbox for tree-level phenomenology," 2013.

[61] F. Staub, "SARAH 4: A tool for (not only SUSY) model builders," 2013.

[62] A. Buckley, J. Butterworth, L. Lonnblad, H. Hoeth, J. Monk, et al., "Rivet user manual," 2010.

[63] "Measurement of the b-tag Efficiency in a Sample of Jets Containing Muons with 5 fb1 of Data from the ATLAS Detector," Tech. Rep. ATLAS-CONF-2012-043, CERN, Geneva, Mar 2012.

[64] "Identification of the Hadronic Decays of Tau Leptons in 2012 Data with the ATLAS Detector," Tech. Rep. ATLASCONF-2013-064, CERN, Geneva, Jul 2013. 